\title{
Unification of Galileon dualities
}

\section{Karol Kampf and Jiří Novotný}

Institute of Particle and Nuclear Physics, Faculty of Mathematics and Physics, Charles University, CZ-18000 Prague, Czech Republic

E-mail: karol.kampf@mff.cuni.cz, jiri.novotny@mff.cuni.cz

ABSTRACT: We study dualities of the general Galileon theory in $d$ dimensions in terms of coordinate transformations on the coset space corresponding to the spontaneously broken Galileon group. The most general duality transformation is found to be determined uniquely up to four free parameters and under compositions these transformations form a group which can be identified with $G L(2, \mathbf{R})$. This group represents a unified framework for all the up to now known Galileon dualities. We discuss a representation of this group on the Galileon theory space and using concrete examples we illustrate its applicability both on the classical and quantum level.

KeYwords: Space-Time Symmetries, Global Symmetries, Scattering Amplitudes

ARXIV EPRINT: 1403.6813 


\section{Contents}

1 Introduction 1

2 Introductory remarks on Galileon in flat space $\quad 2$

2.1 The Galileon Lagrangian 2

2.2 The Feynman rules and tree-level amplitudes 4

3 Coset construction of the Galileon action 5

3.1 Nonlinear realization of the Galilean symmetry 6

$\begin{array}{lr}3.2 \text { Construction of the invariant Lagrangian } & 8\end{array}$

$\begin{array}{lll}3.3 & \text { Generalized Wess-Zumino-Witten terms } & 10\end{array}$

4 Galileon duality as a coset coordinate transformation 11

$5 G L(2, R)$ group of the Galileon dualities $\quad 14$

$\begin{array}{lll}5.1 & \text { Special cases } & 15\end{array}$

$\begin{array}{lll}5.2 & \text { Duality invariants } & 18\end{array}$

6 Applications $\quad 20$

$\begin{array}{lll}\text { 6.1 Classical solutions } & 21\end{array}$

6.1.1 Cylindrically symmetric static solution 23

$\begin{array}{lll}6.1 .2 & \text { Point-like source } & 27\end{array}$

$\begin{array}{lll}6.2 & \text { Duality of classical observables } & 29\end{array}$

$\begin{array}{lll}6.3 & \text { Fluctuations of classical solutions } & 30\end{array}$

6.3.1 Fluctuations of the plane wave background 34

6.3.2 Fluctuations of the cylindrically symmetric solution 40

6.4 Hidden symmetries $\quad 42$

6.5 Duality of the $S$ matrix $\quad 45$

6.6 Tree level amplitudes 48

6.7 Classification of the Galileon theories 52

6.7.1 Galileons in $d=4 \quad 52$

6.7.2 Galileons in $d=3 \quad 54$

6.8 Counterterms 54

$\begin{array}{lll}6.9 & \text { Examples of one-loop order duality } & 57\end{array}$

$\begin{array}{lll}7 & \text { Summary } & 60\end{array}$

A Bottom up construction of the duality subgroup $\alpha_{D}(\theta) \quad 62$

B Compatibility of duality and IHC constraint 64

C Remark on dual observables in the linearized fluctuation theories $\quad 65$ 


\section{Introduction}

The Galileons represent a theory of real scalar field $\phi$ with derivative interactions having number of interesting properties. It emerges in its simplest form as an effective theory of the Dvali-Gabadadze-Porrati model [1,2] as well as of the de Rham-Gabadadze-Tolley massive gravity theory [3] in the decoupling limit. Generalization of the Galileon Lagrangian was proposed by Nicolis, Rattazzi and Trincherini [4] as the long distance modifications of General relativity. In the seminal paper [4] also the complete classification of possible terms of the Galileon Lagrangian has been made and some of the physical consequences have been studied in detail, i.a. it was demonstrated that such theories exhibit the so-called Vainshtein mechanism [5]. In fact the general structures appearing in the Galileon Lagrangian have been already discovered in the 70' as a building blocks of the Horndeski Lagrangian [6], which is the most general Lagrangian built from no more than the second order derivatives of the scalar field and leading to the second-order Euler-Lagrange equations. Generalization of such Lagrangians to curved backgrounds and arbitrary $p$-form fields has been studied in $[7,8]$. From another point of view the Galileon Lagrangian can be obtained as a special non-relativistic limit of the Dirac-Born-Infeld Lagrangian describing the fluctuations of the $d$-dimensional brane in the $d+1$ dimensional space-time [9]. For a pedagogical introduction into the Galileon physics as well as for the complete list of literature see e.g. [10].

Putting aside very important cosmological aspects, the Galileon theory itself has an amazing structure which has been studied intensively in the literature (for pedagogical introductions into the technical aspects see e.g. [11, 12]). For instance on the quantum level it exhibits the so-called non-renormalization theorem which prevents the tree-level Galileon couplings from obtaining the quantum corrections stemming from loops [13-16]. Another interesting feature is the existence of dualities, i.e. such transformations of fields and coordinates which preserve the form of the Galileon Lagrangian, though it changes its couplings. The duality transformations therefore interrelate different Galileon theories on the contrary to the symmetry transformations which leave the action invariant. The first such a duality has been recognized already in the paper [4] where it was shown that the transformation $\phi \rightarrow \phi+\frac{1}{4} H^{2} x^{2}$ converts one form of the Lagrangian into another one. The latter then describes the fluctuations of the Galileon field about the de Sitter background solution. Another example of duality was mentioned and studied in [11] and it corresponds to the dual Legendre transform of the field. The most interesting duality 
has been discovered in [17] in the context of massive gravity and bigravity and has been further studied in [18].

In this paper we study these dualities from the unified point of view. We make use of the fact that the general Galileon theory can be understood as a low-energy effective theory describing the Goldstone bosons corresponding to the spontaneously broken symmetry according to the pattern $G A L(d, 1) \rightarrow I S O(d-1,1)$ where $G A L(d, 1)$ is the so-called Galileon group and its Lagrangian can be identified with generalized Wess-Zumino-Witten terms [19]. This allows us to classify the most general duality transformation and identify it as a non-linear coordinate transformations on the coset space $G A L(d, 1) / S O(d-1,1)$. As we will show such duality transformations form a four-parametric group which can be identified with $G L(2, \mathbf{R})$ and which contains all the above mentioned dualities as special cases. We will also study the representation of this duality group on the Galileon theory space and give examples of physical applications of the duality. Namely we discuss the duality of classical covariant phase spaces and corresponding observables, the duality of fluctuations on the the classical background, the dual realization of the symmetries, the duality of the $S$ matrix and its applications on the tree and one-loop level. We also classify the Galileon theories with respect to the duality generated with specific subgroup of $G L(2, \mathbf{R})$ which leaves the $S$ matrix invariant or under which the tree-level amplitudes trivially scale. We illustrate most of the above topics by means of explicit examples.

This paper is organized as follows. First, in section 2 we introduce the Galileon symmetry and Lagrangian, discuss the Feynman rules and as an illustration we calculate the tree-level amplitudes up to the five-point one. In section 3 we review the coset construction of the Galileon Lagrangian. Section 4 and 5 contain the main results of this work. In section 4 we construct the most general duality transformations and in section 5 we discuss their group structure. Several applications then follow in section 6. Some technical details and alternative approaches are postponed in appendices.

\section{Introductory remarks on Galileon in flat space}

In this section we fix our notation and introduce the classical Galileon Lagrangian. Also some formulae which will be useful in the next sections are presented. We also explicitly evaluate the Feynman rules and as a motivation we calculate the tree-level scattering amplitudes up to five particles in the in and out states.

\subsection{The Galileon Lagrangian}

The Galileon represents the most general theory of a real scalar field $\phi$ in flat $d-$ dimensional space-time the action $S[\phi]$ of which is invariant with respect to the Galilean symmetry

$$
\delta_{a, b} \phi=a+b \cdot x,
$$

where $a$ and $b_{\mu}$ are real parameters. Therefore the Galileon Lagrangian $\mathcal{L}_{G}$ changes under this symmetry at most by a total derivative

$$
\delta_{a, b} \mathcal{L}_{G}=\partial \cdot V_{a, b}
$$


At the quantum level the Lagrangian can be written in the general form

$$
\mathcal{L}_{G}=\mathcal{L}+\mathcal{L}_{C T}
$$

where $\mathcal{L}$ is the leading (classical) part and $\mathcal{L}_{C T}$ corresponds to the higher order counterterms needed for a consistent perturbative calculation of the quantum corrections. The latter part of the Lagrangian will be discussed in more details in section 6.8 , here we concentrate on the leading part $\mathcal{L}$. This can be determined uniquely (up to $d+1$ arbitrary coupling constants) by a second requirement demanding that the classical equations of motion corresponding to $\mathcal{L}$ contain at most second order derivatives of the field. As it has been proven in the seminal paper [4], (see also [11] and [12] for detailed pedagogical introduction and many useful formulae), these conditions allow just $d+1$ possible terms in the Lagrangian

$$
\mathcal{L}=\sum_{n=1}^{d+1} d_{n} \mathcal{L}_{n}=\sum_{n=1}^{d+1} d_{n} \phi \mathcal{L}_{n-1}^{\mathrm{der}}
$$

where $d_{n}$ are real coupling constants and $\mathcal{L}_{n}^{\text {der }}$ can be constructed from $d$-dimensional Levi-Civita tensor $\varepsilon^{\mu_{1} \ldots \mu_{d}}$, the flat-space metric tensor $\eta_{\mu \nu}$ and the matrix of the second derivatives of the field $\partial \partial \phi$ as follows ${ }^{1}$

$$
\mathcal{L}_{n}^{\text {der }}=\varepsilon^{\mu_{1} \ldots \mu_{d}} \varepsilon^{\nu_{1} \ldots \nu_{d}} \prod_{i=1}^{n} \partial_{\mu_{i}} \partial_{\nu_{i}} \phi \prod_{j=n+1}^{d} \eta_{\mu_{j} \nu_{j}}=(-1)^{d-1}(d-n) ! \operatorname{det}\left\{\partial^{\nu_{i}} \partial_{\nu_{j}} \phi\right\}_{i, j=1}^{n}
$$

In four dimensions we have explicitly ${ }^{2}$

$$
\begin{aligned}
\mathcal{L}_{0}^{\text {der }}= & -4 ! \\
\mathcal{L}_{1}^{\text {der }}= & -6 \square \phi \\
\mathcal{L}_{2}^{\text {der }}= & -2\left[(\square \phi)^{2}-\partial \partial \phi: \partial \partial \phi\right] \\
\mathcal{L}_{3}^{\text {der }}= & -\left[(\square \phi)^{3}+2 \partial \partial \phi \cdot \partial \partial \phi: \partial \partial \phi-3 \square \phi \partial \partial \phi \cdot \partial \partial \phi\right] \\
\mathcal{L}_{4}^{\text {der }}= & -\left[(\square \phi)^{4}-6(\square \phi)^{2} \partial \partial \phi: \partial \partial \phi+8 \square \phi \partial \partial \phi \cdot \partial \partial \phi: \partial \partial \phi\right. \\
& \left.-6 \partial \partial \phi \cdot \partial \partial \phi \cdot \partial \partial \phi: \partial \partial \phi+3(\partial \partial \phi: \partial \partial \phi)^{2}\right] .
\end{aligned}
$$

The equation of motion is then

$$
\frac{\delta S[\phi]}{\delta \phi}=\sum_{n=1}^{d+1} n d_{n} \mathcal{L}_{n-1}^{\mathrm{der}}=0
$$

and involves just the second derivatives of the Galileon field.

\footnotetext{
${ }^{1}$ We use the convention $\eta_{\mu \nu}=\operatorname{diag}(1,-1, \ldots,-1), \varepsilon^{0,1, \ldots, d-1}=1$.

${ }^{2}$ Here (and in what follows) we use condensed notation where the dot means contraction of the adjacent Lorentz indices, e.g.

$$
\partial \partial \phi \cdot \partial \partial \phi: \partial \partial \phi=\partial_{\mu} \partial_{\sigma} \phi \cdot \partial^{\sigma} \partial_{\nu} \phi: \partial^{\mu} \partial^{\nu} \phi
$$
}


Let us note that the operator basis $\mathcal{L}_{n}$ is not unique, we can choose also another set which differs from (2.5) by a total derivative and possible re-scaling. One of the many equivalent forms of the Lagrangian which can be obtained from (2.5) by means of the integration by parts and simple algebra is

$$
\widetilde{\mathcal{L}}=\sum_{n=1}^{d+1} c_{n} \widetilde{\mathcal{L}}_{n}=\sum_{n=1}^{d+1} c_{n}(\partial \phi \cdot \partial \phi) \mathcal{L}_{n-2}^{\mathrm{der}}
$$

Let us mention useful formulae (for derivation see e.g. [12])

$$
(\partial \phi \cdot \partial \phi) \mathcal{L}_{n-1}^{\text {der }}=-\frac{2(d-n+1)}{n+1}\left[\phi \mathcal{L}_{n}^{\text {der }}-\partial_{\mu}\left(H_{n}^{\mu}+\frac{n-1}{d-n+1} G_{n}^{\mu}\right)\right]
$$

where

$$
\begin{aligned}
& H_{n}^{\mu}=\phi \partial_{\nu_{1}} \phi \varepsilon^{\mu \mu_{2} \ldots \mu_{d}} \varepsilon^{\nu_{1} \ldots \nu_{d}} \prod_{i=2}^{n} \partial_{\mu_{i}} \partial_{\nu_{i}} \phi \prod_{j=n+1}^{d} \eta_{\mu_{j} \nu_{j}} \\
& G_{n}^{\mu}=\frac{1}{2}(\partial \phi \cdot \partial \phi) \partial^{\mu} \phi \varepsilon^{\mu_{2} \ldots \mu_{n} \alpha_{1} \ldots \alpha_{d-n+1}} \varepsilon^{\nu_{2} \ldots \nu_{n} \beta_{1} \ldots \beta_{d-n+1}} \prod_{j=1}^{d-n+1} \eta_{\alpha_{j} \beta_{j}} \prod_{k=2, k \neq i}^{n} \partial_{\mu_{k}} \partial_{\nu_{k}} \phi .
\end{aligned}
$$

and thus after integration and omitting the surface terms we get

$$
\int \mathrm{d}^{d} x(\partial \phi \cdot \partial \phi) \mathcal{L}_{n-1}^{\mathrm{der}}=-\int \mathrm{d}^{d} x \frac{2(d-n+1)}{n+1} \phi \mathcal{L}_{n}^{\mathrm{der}} .
$$

\subsection{The Feynman rules and tree-level amplitudes}

For further convenience let us also write down explicitly the Feynman rule for $n$-point vertex

$$
\mathcal{V}_{n}(1,2, \ldots, n)=(-1)^{n} d_{n}(d-n+1) !(n-1) ! \sum_{\sigma \in Z_{n}} G\left(p_{\sigma(1)}, p_{\sigma(2)}, \ldots, p_{\sigma(n-1)}\right),
$$

where we have introduced the Gram determinant $G\left(p_{1}, \ldots, p_{n-1}\right)$

$$
G\left(p_{1}, \ldots, p_{n-1}\right)=-\frac{1}{(d-n+1) !} \varepsilon^{p_{1} \ldots p_{n-1} \mu_{n} \ldots \mu_{d}} \varepsilon^{p_{1}, \ldots, p_{n-1} \nu_{n} \ldots \nu_{d}} \prod_{j=n}^{d} \eta_{\mu_{j} \nu_{j}}
$$

and where the sum is over the cyclic permutations only. ${ }^{3}$

Using this Feynman rules, one can in principle calculate any tree-level $n$-point amplitude in the pure Galileon theory. What it means for $n=3,4,5$ in the language of Feynman diagrams is depicted in figure 1. Note that crossing is tacitly assumed for these graphs which finally leads to four diagrams for 4-pt scattering and 26 for 5-pt scattering. However, due to the complicated structure of the vertices the evaluation of the individual contributions of the Feynman graphs is not an easy task. The most economic way how to

\footnotetext{
${ }^{3}$ Note that, the Gram determinant is independent on the ordering of the vector arguments.
} 

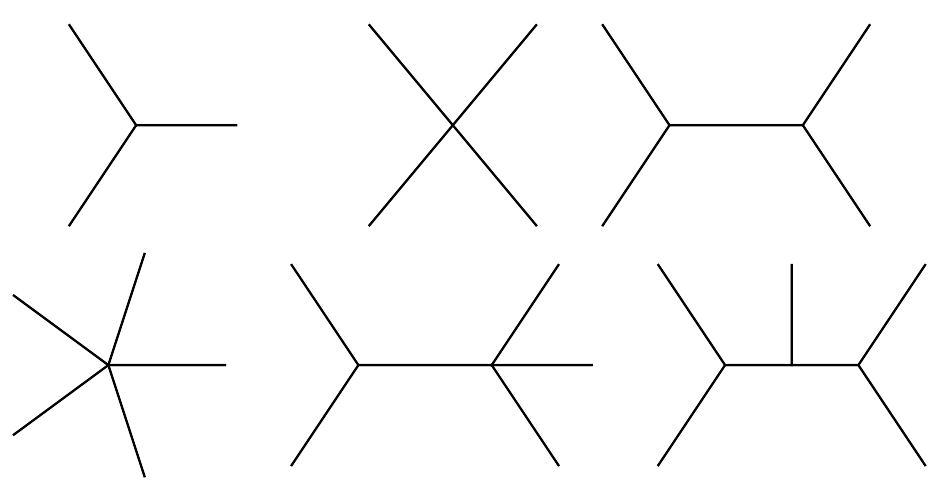

Figure 1. The topologies of Feynman diagrams at the tree-level for the three-point, four-point (first line) and five-point (second line) Galileon scattering amplitudes.

organize the rather lengthy and untransparent calculation is the machinery of the BerendsGiele like recursion relations ${ }^{4}[21]$ which allows for an efficient computer algoritmization of the problem. In four dimension (in the theory without tadpole and with canonical kinetic term, i.e. with $d_{1}=0$ and $d_{2}=1 / 12$ ) we get to the following results

$$
\begin{aligned}
\mathcal{M}(1,2,3) & =6 d_{3} G(1,2)=\frac{3}{2} d_{3} p_{3}^{4}=0 \\
\mathcal{M}(1,2,3,4) & =12\left(2 d_{4}-9 d_{3}^{2}\right) G(1,2,3) \\
\mathcal{M}(1,2,3,4,5) & =-24\left(72 d_{3}^{3}-24 d_{3} d_{4}+5 d_{5}\right) G(1,2,3,4)
\end{aligned}
$$

(we were also capable to calculate the 6-pt diagrams which involves 235 Feynman diagrams). Without the deeper understanding of the structure of the Galileon theory these results look suspiciously simple $;^{5}$ in fact it was our main motivation for starting to study this model more systematically. In what follows we shall i.a. show how to understand these results and how they can be obtained almost without calculation on a single sheet of paper.

\section{Coset construction of the Galileon action}

The Galileon field can be also interpreted as a Goldstone boson corresponding to the spontaneously broken Galileon symmetry [19]. Therefore, to obtain the most general Lagrangian for the Galileon, the general theory of nonlinear realization [22-25] should be used. Because the localized Galilean symmetries are non independent, the number of Goldstone bosons is not equal to the number of the broken generators and an additional constraint known as the inverse Higgs constraint [26] has to be introduced. However, as discussed in [19], only the counterterm part $\mathcal{L}_{C T}$ can be obtained in this way. The classical Galileon Lagrangian $\mathcal{L}$ is in fact invariant only up to the total derivative and the corresponding action represents an analogue of the Wess-Zumino-Witten term [27-30] originally known from the effective low

\footnotetext{
${ }^{4}$ For an application to a similar problem see e.g. [20].

${ }^{5}$ Note that, while the four- and five-point amplitudes are sums of Feynman graphs including those with one and two propagators naively generating pole terms (see figure 1), the resulting amplitude is represented by a purely contact term.
} 
energy theory of QCD. Such a term of the action can be reconstructed from its variation and is thus expressed as $d+1$ dimensional integral. In this section we give a brief review of the coset construction based on the nonlinearly realized Galileon symmetry and of the interpretation of the classical galileon Lagrangian as the generalized Wess-Zumino-Witten term. Further details and generalizations can be found in the original paper [19].

\subsection{Nonlinear realization of the Galilean symmetry}

The Galilean symmetry is a prominent example of the so called non-uniform symmetry, i.e. a symmetry which does not commute with the space-time translations [31, 32]. Indeed, denoting the infinitesimal translations and Galilean transformations of the Galileon field $\delta_{c} \phi$ and $\delta_{a, b} \phi$ respectively,

$$
\begin{aligned}
\delta_{c} \phi & =c \cdot \partial \phi \\
\delta_{a, b} \phi & =a+b \cdot x,
\end{aligned}
$$

we get

$$
\left[\delta_{c}, \delta_{a, b}\right] \phi=c \cdot b=\delta_{c \cdot b, 0} \phi
$$

Let us add to this transformations also the Lorentz rotations and boosts $\delta_{\omega}$

$$
\delta_{\omega} \phi=\frac{1}{2} \omega^{\mu \nu}\left(x_{\mu} \partial_{\nu}-x_{\nu} \partial_{\mu}\right) \phi
$$

we get then

$$
\left[\delta_{\omega}, \delta_{a, b}\right] \phi=-b \cdot \omega \cdot x=\delta_{0,-b \cdot \omega} \phi=\delta_{0, \omega \cdot b} \phi
$$

Therefore the infinitesimal transformations $\delta_{c}, \delta_{\omega}, \delta_{a, b}$ form a closed algebra with generators $P_{a}, J_{a b}=-J_{b a}, A$ and $B_{a}$ respectively. In terms of these generators

$$
\begin{aligned}
\delta_{c} & =-\mathrm{i} c^{a} P_{a} \\
\delta_{\omega} & =-\frac{\mathrm{i}}{2} \omega^{a b} J_{a b} \\
\delta_{a, b} & =-\mathrm{i} a A-\mathrm{i} b^{a} B_{a}
\end{aligned}
$$

and the commutator algebra can be rewritten in the form of the Galileon algebra $\mathfrak{g a l}(d, 1)$

$$
\begin{aligned}
{\left[P_{a}, P_{b}\right] } & =\left[P_{a}, A\right]=\left[B_{a}, A\right]=\left[J_{a b}, A\right]=0 \\
{\left[P_{a}, B_{b}\right] } & =\mathrm{i} \eta_{a b} A \\
{\left[J_{a b}, P_{c}\right] } & =\mathrm{i}\left(\eta_{b c} P_{a}-\eta_{a c} P_{b}\right) \\
{\left[J_{a b}, B_{c}\right] } & =\mathrm{i}\left(\eta_{b c} B_{a}-\eta_{a c} B_{b}\right) \\
{\left[J_{a b}, J_{c d}\right] } & =\mathrm{i}\left(\eta_{b c} J_{a d}+\eta_{a d} J_{b c}-\eta_{a c} J_{b d}-\eta_{b d} J_{a c}\right)
\end{aligned}
$$

which corresponds to the Galileon group $G A L(d, 1)$ (see [19]).

Within the Galileon theory this group is realized non-linearly on the fields $\phi$ and space-time coordinates $x^{\mu}$. Indeed, for the generators $P_{a}, A$ a $B_{a}$ we have

$$
\begin{aligned}
-\mathrm{i} P_{a} x^{\mu} & =\delta_{a}^{\mu} \\
-\mathrm{i} A \phi & =1 \\
-\mathrm{i} B_{a} \phi & =x_{a} .
\end{aligned}
$$


The generators $A$ a $B_{a}$ are spontaneously broken, the order parameter can be identified with

$$
\left\langle 0\left|\delta_{a, b} \phi\right| 0\right\rangle=a+b \cdot x .
$$

This corresponds to the symmetry breaking pattern $G A L(d, 1) \rightarrow I S O(d-1,1)$. Let us note that the above transformations are not completely independent in the sense of refs. [33, 32]. Indeed, their localized forms with space-time dependent parameters $a(x)$ and $b_{\mu}(x)$ yield the same local transformation

$$
\delta_{a(x), b(x)} \phi=a(x)+b(x) \cdot x
$$

which corresponds to the local shift of the Galileon field $\phi$. More precisely, writing $a_{b}(x)=$ $x \cdot b(x)$, we can identify

$$
\delta_{a_{b}(x), 0}=\delta_{0, b(x)} .
$$

Physically this means that the local fluctuations of the order parameter which correspond to the Goldstone modes are not independent. As a result the particle spectrum does not contain the same number of Goldstone bosons as is the number of the broken generators (i.e. $d+1$ ) but just one zero mass mode which can be identified with the Galileon field $\phi$. (see $[32,34]$ for recent discussion of this issue).

Construction of the low energy effective Lagrangian describing the dynamics of the Goldstone bosons corresponding to the spontaneous breakdown of the non-uniform symmetries is a generalization of the coset construction of Callan, Coleman, Wess and Zumino [22, 23] and has been formulated by Volkov [24] and Ogievetsky [25]. Applied to the Galileon case, where the only linearly realized generators of the Galileon group are the Lorentz rotations and boosts $J_{a b}$, the coset space is $G A L(d, 1) / S O(d-1,1)$ the elements of which are the left cosets $\{g S O(d-1,1)\}$ where $g \in G A L(d, 1)$. The coordinates on this coset space can be chosen in a standard way by means of a unique choice of the representant $U$ of each left coset. Such a representant can be written in terms of the coset coordinates $x^{a}, \phi$ and $L^{a}$ as

$$
U \equiv U(x, \phi, L)=\exp \left(\mathrm{i} x^{a} P_{a}\right) \exp \left(\mathrm{i} \phi A+\mathrm{i} L^{a} B_{a}\right)
$$

The general element of the galileon group $g \in G A L(d, 1)$

$$
g=\exp \left(\frac{\mathrm{i}}{2} \omega^{a b} J_{a b}\right) \exp \left(\mathrm{i} c^{a} P_{a}\right) \exp \left(\mathrm{i} a A+\mathrm{i} b^{a} B_{a}\right)
$$

acts on the cosets by means of the left multiplication and consequently the coset coordinates transform according to

$$
U^{\prime} \equiv U\left(x^{\prime}, \phi^{\prime}, L^{\prime}\right)=g U h^{-1}
$$

where $h \equiv h(g, x, \phi, L) \in S O(d-1,1)$ is the compensator arranging $U^{\prime}$ to be of the form (3.13). As usual, the stability group, which is the Lorentz group $S O(d-1,1)$ here, is realized linearly ( $\phi$ transformed as a scalar and $x$ and $L$ are vectors), and the general element (3.14) of the Galileon group $g \in G A L(d, 1)$ acts on $U$ as follows

$$
g U(x, \phi, L)=\exp \left(\mathrm{i} x^{a \prime} P_{a}\right) \exp \left(\mathrm{i} \phi^{\prime} A+\mathrm{i} L^{a \prime} B_{a}\right) \exp \left(\frac{\mathrm{i}}{2} \omega^{a b} J_{a b}\right)
$$


where

$$
x^{a \prime}=\Lambda(\omega)_{b}^{a}\left(x^{b}+c^{b}\right), \quad \phi^{\prime}=\phi+a+b \cdot x, \quad L^{a \prime}=\Lambda(\omega)_{b}^{a}\left(L^{b}+b^{b}\right)
$$

and where

$$
\Lambda(\omega)=\exp \left(\frac{1}{2} \omega^{a b} M_{a b}\right), \quad\left(M_{a b}\right)_{d}^{c}=\delta_{a}^{c} \eta_{b d}-\delta_{b}^{c} \eta_{a d} .
$$

As a result, for the general element of the Galileon group $g \in G A L(d, 1)$ we have the following compensator

$$
h(g, x, \phi, L)=\exp \left(\frac{\mathrm{i}}{2} \omega^{a b} J_{a b}\right) .
$$

Note that, the compensator does not depend on the coset coordinates $(x, \phi, L)$ and therefore treating $\phi$ and $L^{a}$ as space-time dependent fields, the compensator has no explicit or implicit $x$ dependence. This simplifies the application of the general recipe [24, 25] significantly, because the requirement of the invariance with respect to the local stability group can be replaced by much simpler requirement of global invariance.

\subsection{Construction of the invariant Lagrangian}

The basic object for the construction of the effective Lagrangian is the Maurer-Cartan form, which can be expressed in the coordinates $x^{a}, \phi$ and $L^{a}$ as

$$
\begin{aligned}
\frac{1}{\mathrm{i}} U^{-1} \mathrm{~d} U & =\exp \left(-\mathrm{i} \phi A-\mathrm{i} L^{b} B_{b}\right) \exp \left(-\mathrm{i} x^{d} P_{d}\right) \mathrm{d}\left(\exp \left(\mathrm{i} x^{c} P_{c}\right) \exp \left(\mathrm{i} \phi A+\mathrm{i} L^{a} B_{a}\right)\right) \\
& =\exp \left(-\mathrm{i} L^{b} B_{b}\right)\left(\mathrm{d} x^{c} P_{c}+\mathrm{d} \phi A+\mathrm{d} L^{d} B_{d}\right) \exp \left(\mathrm{i} L^{a} B_{a}\right)
\end{aligned}
$$

where in the second line we have used the fact that $A$ commutes with all the other generators. Using further

$$
\exp \left(-\mathrm{i} L^{b} B_{b}\right) P_{c} \exp \left(\mathrm{i} L^{a} B_{a}\right)=P_{c}-L^{b} \eta_{b c} A
$$

we get finally

$$
\begin{aligned}
\frac{1}{\mathrm{i}} U^{-1} \mathrm{~d} U & =\mathrm{d} x^{c} P_{c}+\left(\mathrm{d} \phi-L^{a} \eta_{b c} \mathrm{~d} x^{c}\right) A+\mathrm{d} L^{d} B_{d} \\
& \equiv \omega_{P}^{c} P_{c}+\omega_{A} A+\omega_{B}^{d} B_{d}
\end{aligned}
$$

The form $\omega_{P}^{c}$ is particularly simple. In the general case we get $\omega_{P}^{a}=e_{\mu}^{a}(x) \mathrm{d} x^{\mu}$ and $e_{\mu}^{a}$ plays a role of $d$-bein, intertwining the abstract group indices $a, \ldots$ with space-time indices $\mu, \ldots$ and the flat metric $\eta_{a b}$ with the effective space-time metric $g_{\mu \nu}$ according to

$$
g_{\mu \nu}=\eta_{a b} e_{\mu}^{a} e_{\nu}^{b}
$$

In our case $e_{\mu}^{a}=\delta_{\mu}^{a}$, the space-time metric is therefore flat

$$
g_{\mu \nu}=\eta_{\mu \nu}
$$

and the abstract group indices are identical with the space-time ones. This also ensures that the volume element $\mathrm{d}^{d} x$ is invariant with respect to the non-linearly realized Galileon 
group. Note also that there is no term of the form $\omega_{J}^{a b} J_{a b}$ on the right hand side of (3.20). This implies that the usual group covariant derivative is in our case identical with ordinary partial derivatives $\partial_{\alpha}$. The forms $\omega_{P}^{c}, \omega_{A}$ and $\omega_{B}^{d}$ transform under a general element of the Galileon (3.14) group $g \in G A L(d, 1)$ (cf. (3.17)) according to

$$
\begin{aligned}
\omega_{P}^{\prime a} & =\Lambda(\omega)_{b}^{a} \omega_{P}^{b} \\
\omega_{B}^{\prime a} & =\Lambda(\omega)_{b}^{a} \omega_{B}^{b} \\
\omega_{A}^{\prime} & =\omega_{A} .
\end{aligned}
$$

These forms span three irreducible representations of the stability group $S O(d-1,1)$ (namely two vectors and one scalar) and can be therefore used separately as the basic building blocks for the construction of the effective Lagrangian. The general recipe requires to use this building block and their (covariant) derivatives to construct all the possible terms which are invariant with respect to local stability group. As we have mentioned above, in our case we make do with ordinary partial derivatives and the last requirement can be rephrased as the global $S O(d-1,1)$ invariance when we identify the abstract group and space-time indices with help of the trivial $d$-bein $\delta_{\mu}^{a}$. Therefore, writing

$$
\mathrm{d} \phi(x)=\partial_{\mu} \phi(x) \mathrm{d} x^{\mu}, \quad \mathrm{d} L^{\nu}(x)=\partial_{\mu} L^{\nu}(x) \mathrm{d} x^{\mu},
$$

the most general invariant term of the Lagrangian is the Lorentz invariant combinations of the fields $\partial_{\mu} L^{\nu}$ and $D_{\mu} \phi$, where

$$
D_{\mu} \phi \equiv \partial_{\mu} \phi-L_{\mu}
$$

and their derivatives.

Apparently we have ended up with $d+1$ Goldstone fields $\phi$ and $L_{\mu}$ however this is not the final answer. In fact these fields are not independent. The standard possibility how to eliminate the unwanted degrees of freedom is to require an additional constraint $[19,32]$

$$
\omega_{A}=0 \Longleftrightarrow L_{\mu}=\partial_{\mu} \phi
$$

which is invariant with respect to the group $G A L(d, 1)$ and which is known as the inverse Higgs constraint (IHC) [26]. Then the only remaining nontrivial building blocks are $\partial_{\mu} \partial_{\nu} \phi$ and its derivatives ${ }^{6}$ and the general Lagrangian is

$$
\mathcal{L}_{\text {inv }}=\mathcal{L}_{\text {inv }}\left(\partial_{\mu} \partial_{\nu} \phi, \partial_{\lambda} \partial_{\mu} \partial_{\nu} \phi, \ldots\right) .
$$

\footnotetext{
${ }^{6}$ Another possibility how to treat the problem of additional degrees of freedom is based on the field redefinition

$$
L^{\mu}=\psi^{\mu}+\partial_{\mu} \phi
$$

where $\psi^{\mu}$ are new fields. Then

$$
\mathcal{L}\left(\partial_{\mu} L^{\nu}, D_{\mu} \phi\right)=\mathcal{L}\left(\partial_{\mu} L^{\nu}, \partial_{\mu} \phi-L_{\mu}\right)=\mathcal{L}\left(\partial_{\mu} \psi^{\nu}+\partial_{\mu} \partial^{\nu} \phi, \psi^{\mu}\right)
$$

The invariant term $M^{2} D_{\mu} \phi D^{\mu} \phi$, which was responsible for the kinetic term of the field $\phi$ in the original Lagrangian goes within the new parametrization in terms of $\phi$ and $\psi^{\mu}$ into the mass term of the field $\psi^{\mu}$. This field then does not correspond more to the Goldstone boson and can be integrated out from the effective Lagrangian, provided we are interested in the dynamics of the field $\phi$ only. We end up again with the just one nontrivial building block $\partial_{\mu} \partial_{\nu} \phi$. See [32] for detailed discussion of this aspect of the spontaneously broken non-uniform symmetries.
} 


\subsection{Generalized Wess-Zumino-Witten terms}

The Galileon Lagrangian represents a different type of possible terms contributing to the invariant action, namely those which are not strictly invariant on the Lagrangian level, but are invariant only up to a total derivative. Such terms can be identified as the generalized Wess-Zumino-Witten (WZW) terms [27, 28], as was proved and discussed in detail in [29, 30]. From the point of view of the coset construction, the WZW terms originate in the integrals of the closed invariant $(d+1)$-forms ${ }^{7} \omega_{d+1}$ on $G A L(d, 1) / S O(d-1,1)$ (these correspond to the variation of the action) over the $d+1$ dimensional ball $B_{d+1}$ the boundary of which is the compactified space-time $S_{d}=\partial B_{d+1}$

$$
S^{W Z W}=\int_{B_{d+1}} \omega_{d+1}
$$

In order to prevent these contributions to the action to degenerate into the strictly invariant Lagrangian terms discussed above it is necessary that the form $\omega_{d+1}$ is not an exterior derivative of the invariant $d$-form on $G A L(d, 1) / S O(d-1,1)$. This means that $\omega_{d+1}$ has to be a nontrivial element of the cohomology $H^{d+1}(G A L(d, 1) / S O(d-1,1), \mathbf{R})$ (see [35] and also [36] for a recent review on this topic). In the case of Galileon such forms can be constructed out of the covariant 1-forms $\omega_{P}^{\mu}, \omega_{A}$ a $\omega_{B}^{\mu}$ with indices contracted appropriately to get Lorentz invariant combinations. As was shown in [19], there are $d+1$ such $\omega_{d+1}$, namely

$$
\begin{aligned}
\omega_{d+1}^{(n)} & =\varepsilon_{\mu_{1} \ldots \mu_{d}} \omega_{A} \wedge \omega_{B}^{\mu_{1}} \wedge \ldots \wedge \omega_{B}^{\mu_{n-1}} \wedge \omega_{P}^{\mu_{n}} \wedge \ldots \wedge \omega_{P}^{\mu_{d}} \\
& =\varepsilon_{\mu_{1} \ldots \mu_{d}}\left(\mathrm{~d} \phi-L_{\mu} \mathrm{d} x^{\mu}\right) \wedge \mathrm{d} L^{\mu_{1}} \wedge \ldots \wedge \mathrm{d} L^{\mu_{n-1}} \wedge \mathrm{d} x^{\mu_{n}} \wedge \ldots \wedge \mathrm{d} x^{\mu_{d}},
\end{aligned}
$$

where $n=1,2, \ldots, d+1$. These forms are closed

$$
\omega_{d+1}^{(n)}=\mathrm{d} \beta_{d}^{(n)}
$$

where $^{8}[19]$

$$
\begin{aligned}
\beta_{d}^{(n)}= & \varepsilon_{\mu_{1} \ldots \mu_{d}} \phi \mathrm{d} L^{\mu_{1}} \wedge \ldots \wedge \mathrm{d} L^{\mu_{n-1}} \wedge \mathrm{d} x^{\mu_{n}} \wedge \ldots \wedge \mathrm{d} x^{\mu_{d}} \\
& +\frac{n-1}{2(d-n+2) !} \varepsilon_{\mu_{1} \ldots \mu_{d}} L^{2} \mathrm{~d} L^{\mu_{1}} \wedge \ldots \wedge \mathrm{d} L^{\mu_{n-2}} \wedge \mathrm{d} x^{\mu_{n-1}} \wedge \ldots \wedge \mathrm{d} x^{\mu_{d}}
\end{aligned}
$$

and therefore

$$
\int_{B_{d+1}} \omega_{d+1}^{(n)}=\int_{\partial B_{d+1}} \beta_{d}^{(n)}=\int_{S_{d}} \beta_{d}^{(n)}
$$

Note that the $d$-forms $\beta_{d}^{(n)}$ are not invariant, and therefore $\omega_{d+1}^{(n)}$ are nontrivial elements of $H^{d+1}(G A L(d, 1) / S O(d-1,1), \mathbf{R})$. Imposing now the IHC constraint $(3.25)$, we can finally identify

$$
\int_{S_{d}} \beta_{d}^{(n)}=\frac{1}{n} \int_{S_{d}} \mathrm{~d}^{d} x \mathcal{L}_{n}
$$

\footnotetext{
${ }^{7}$ More precisely we integrate the pull-back of the form $\omega_{d+1}$ with respect to the map $B_{d+1} \rightarrow$ $G A L(d, 1) / S O(d-1,1)$ which maps $\left(x^{\mu}, x^{d}\right) \rightarrow\left(\delta_{\mu}^{a} x^{\mu}, \phi\left(x^{\mu}, x^{d}\right), L^{\mu}\left(x^{\mu}, x^{d}\right)\right)$.

${ }^{8}$ The opposite sign of the second term in comparison with [19] stems from different convention for the metric tensor $\eta_{\mu \nu}$.
} 
The classical Galileon action is therefore a linear combination of the generalized WessZumino-Witten terms corresponding to the $d+1$ elements $\omega_{d+1}^{(n)}=\mathrm{d} \beta_{d}^{(n)}$ of the cohomology $H^{d+1}(G A L(d, 1) / S O(d-1,1), \mathbf{R})$ built with help of the forms $\omega_{P}^{\mu}, \omega_{A}$ a $\omega_{B}^{\mu}$.

\section{Galileon duality as a coset coordinate transformation}

The canonical coordinates $(x, \phi, L)$ on the coset space $G A L(d, 1) / S O(d-1,1)$ which we have defined according to (3.13) are not the only possible ones. We can freely use any other set of coordinates connected with them by a general coordinate transformation of the form

$$
\begin{aligned}
x^{\mu} & =\xi^{\mu}\left(x^{\prime}, L^{\prime}, \phi^{\prime}\right) \\
L^{\mu} & =\Lambda^{\mu}\left(x^{\prime}, L^{\prime}, \phi^{\prime}\right) \\
\phi & =f\left(x^{\prime}, L^{\prime}, \phi^{\prime}\right) .
\end{aligned}
$$

Not all such new coordinates are of any use, e.g. those transformations (4.1) which are not covariant with respect to the $S O(d-1,1)$ symmetry will hide this symmetry in the effective Lagrangian. Even if the covariance is respected, in the general case the resulting Lagrangian might be difficult to recognize as a Galileon theory. In this section we shall make a classification of those coordinate changes which preserve the general form of the Galileon action as a linear combination of the $d+1$ terms discussed in the previous sections (though we allow for change of the couplings). Such a transformation of the coset coordinates can be then interpreted as a Galileon duality.

It is obvious from (3.27) and (3.28) that, provided the forms $\omega_{P}^{\mu}, \omega_{A}$ a $\omega_{B}^{\mu}$ can be expressed in the primed coordinates as a (covariant) linear combination (with constant coefficients) of the primed forms $\omega_{P}^{\prime \mu}, \omega_{A}^{\prime}$ and $\omega_{B}^{\prime \mu}$ where

$$
\begin{aligned}
\omega_{P}^{\prime \mu} & =\mathrm{d} x^{\prime \mu} \\
\omega_{A}^{\prime} & =\mathrm{d} \phi^{\prime}-L_{\mu}^{\prime} \mathrm{d} x^{\prime \mu} \\
\omega_{B}^{\prime \mu} & =\mathrm{d} L^{\prime \mu},
\end{aligned}
$$

the coordinate transformation corresponds to a duality transformation of the Galileon action. Indeed, provided ${ }^{9}$

$$
\begin{aligned}
& \omega_{B}^{\mu}=\alpha_{B B} \omega_{B}^{\prime \mu}+\alpha_{B P} \omega_{P}^{\prime \mu} \\
& \omega_{P}^{\mu}=\alpha_{P B} \omega_{B}^{\prime \mu}+\alpha_{P P} \omega_{P}^{\prime \mu} \\
& \omega_{A}=\alpha_{A A} \omega_{A}^{\prime}
\end{aligned}
$$

we have

$$
\begin{aligned}
\omega_{d+1}^{(n)} & =\varepsilon_{\mu_{1} \ldots \mu_{d}} \omega_{A} \wedge \omega_{B}^{\mu_{1}} \wedge \ldots \wedge \omega_{B}^{\mu_{n-1}} \wedge \omega_{P}^{\mu_{n}} \wedge \ldots \wedge \omega_{P}^{\mu_{d}} \\
& =\alpha_{A A} \sum_{k=0}^{d-n+1} \sum_{l=0}^{n-1}\left(\begin{array}{c}
d-n+1 \\
k
\end{array}\right)\left(\begin{array}{c}
n-1 \\
l
\end{array}\right) \alpha_{P B}^{k} \alpha_{B B}^{l} \alpha_{P P}^{d-n+1-k} \alpha_{B P}^{n-1-l} \omega_{d+1}^{\prime(l+k+1)}
\end{aligned}
$$

\footnotetext{
${ }^{9}$ Note that, the constants $\alpha_{I J}$ cannot be decorated with any Lorentz index because the only invariant tensors at our disposal are $\eta_{\mu \nu}$ and $\varepsilon_{\mu_{1} \ldots \mu_{d}}$.
} 
and, after imposing the IHC constraint ${ }^{10}$ (3.25), the corresponding term in the action satisfies

$$
\int_{S_{d}} \beta_{d}^{(n)}=\alpha_{A A} \sum_{k=0}^{d-n+1} \sum_{l=0}^{n-1}\left(\begin{array}{c}
d-n+1 \\
k
\end{array}\right)\left(\begin{array}{c}
n-1 \\
l
\end{array}\right) \alpha_{P B}^{k} \alpha_{B B}^{l} \alpha_{P P}^{d-n+1-k} \alpha_{B P}^{n-1-l} \int_{S_{d}^{\prime}} \beta_{d+1}^{(l+k+1)} .
$$

This means that the coordinate transformation maps linear combination of the $d+1$ basic building block of the Galileon action onto different linear combination of the same building blocks and the two apparently different Galileon theories are in fact dual to each other.

The conditions (4.3) constraint the form of the duality transformation (4.1) strongly. We have in the primed coordinates (here and in what follows the superscript at the symbol of partial derivative indicates the corresponding primed variable, e.g. $\left.\partial^{(\phi)} \equiv \partial / \partial \phi^{\prime}\right)$

$$
\begin{aligned}
\omega_{P}^{\mu}= & \partial_{\nu}^{(L)} \xi^{\mu} \omega_{B}^{\prime \nu}+\left(\partial_{\nu}^{(x)} \xi^{\mu}+L_{\nu}^{\prime} \partial^{(\phi)} \xi^{\mu}\right) \omega_{P}^{\prime \nu}+\partial^{(\phi)} \xi^{\mu} \omega_{A}^{\prime} \\
\omega_{B}^{\mu}= & \partial_{\nu}^{(L)} \Lambda^{\mu} \omega_{B}^{\prime \nu}+\left(\partial_{\nu}^{(x)} \Lambda^{\mu}+L_{\nu}^{\prime} \partial^{(\phi)} \Lambda^{\mu}\right) \omega_{P}^{\prime \nu}+\partial^{(\phi)} \Lambda^{\mu} \omega_{A}^{\prime} \\
\omega_{A}= & \left(\partial^{\phi} f-\Lambda_{\mu} \partial^{(\phi)} \xi^{\mu}\right) \omega_{A}^{\prime}+\left(\partial_{\mu}^{(L)} f-\Lambda_{\nu} \partial_{\mu}^{(L)} \xi^{\nu}\right) \omega_{B}^{\prime \mu} \\
& +\left[\partial_{\nu}^{(x)} f+L_{\nu}^{\prime} \partial^{(\phi)} f-\Lambda_{\mu}\left(\partial_{\nu}^{(x)} \xi^{\mu}+L_{\nu}^{\prime} \partial^{(\phi)} \xi^{\mu}\right)\right] \omega_{P}^{\prime \nu}
\end{aligned}
$$

and comparing the coefficients at $\omega_{P}^{\prime \nu}, \omega_{A}^{\prime}$ and $\omega_{B}^{\prime \nu}$ in the expressions for $\omega_{P}^{\mu}$ and $\omega_{B}^{\mu}$ with the corresponding right hand sides of (4.3) we get the following set of differential equations for $\xi^{\mu}$ and $\Lambda^{\mu}$

$$
\begin{aligned}
& \partial^{(\phi)} \xi^{\mu}=0, \quad \partial_{\nu}^{(L)} \xi^{\mu}=\delta_{\nu}^{\mu} \alpha_{P B}, \quad \partial_{\nu}^{(x)} \xi^{\mu}+L_{\nu}^{\prime} \partial^{(\phi)} \xi^{\mu}=\delta_{\nu}^{\mu} \alpha_{P P}, \\
& \partial^{(\phi)} \Lambda^{\mu}=0, \quad \partial_{\nu}^{(L)} \Lambda^{\mu}=\delta_{\nu}^{\mu} \alpha_{B B}, \quad \partial_{\nu}^{(x)} \Lambda^{\mu}+L_{\nu}^{\prime} \partial^{(\phi)} \Lambda^{\mu}=\delta_{\nu}^{\mu} \alpha_{B P} .
\end{aligned}
$$

Integration of these equations is trivial, we get (up to the additive constants ${ }^{11}$ )

$$
\begin{aligned}
\xi^{\mu} & =\alpha_{P B} L^{\prime \mu}+\alpha_{P P} x^{\prime \mu} \\
\Lambda^{\mu} & =\alpha_{B B} L^{\prime \mu}+\alpha_{B P} x^{\prime \mu} .
\end{aligned}
$$

Comparison of coefficients in both expressions for $\omega_{A}$ gives, after using the explicit form (4.8) of $\xi^{\mu}$ and $\Lambda^{\mu}$, the following differential equations for $f$

$\partial^{(\phi)} f=\alpha_{A A}, \quad \partial_{\mu}^{(L)} f=\alpha_{P B}\left(\alpha_{B B} L_{\mu}^{\prime}+\alpha_{B P} x_{\mu}^{\prime}\right), \quad \partial_{\nu}^{(x)} f+L_{\nu}^{\prime} \partial^{(\phi)} f=\alpha_{P P}\left(\alpha_{B B} L_{\nu}^{\prime}+\alpha_{B P} x_{\nu}^{\prime}\right)$

From the first equation it follows

$$
f=\alpha_{A A} \phi^{\prime}+F\left(x^{\prime}, L^{\prime}\right)
$$

\footnotetext{
${ }^{10}$ Note that, the formula $\omega_{A}=\alpha_{A A} \omega_{A}^{\prime}$ ensures a compatibility of the IHC constraint with the coordinate transformation.

${ }^{11}$ We have set these additive constants equal to zero. The reason is that, if nonzero, they corresponds to the additional combination of the space-time translation and Galileon transformation. Both these additional contributions are exact symmetries of the Galileon theory and does not bring about anything new.
} 
where the function $F$ of two variables satisfies

$$
\begin{aligned}
& \partial_{\mu}^{(L)} F=\alpha_{P B}\left(\alpha_{B B} L_{\mu}^{\prime}+\alpha_{B P} x_{\mu}^{\prime}\right) \\
& \partial_{\nu}^{(x)} F=\alpha_{P P}\left(\alpha_{B B} L_{\nu}^{\prime}+\alpha_{B P} x_{\nu}^{\prime}\right)-\alpha_{A A} L_{\nu}^{\prime} .
\end{aligned}
$$

Integration of these equations is possible only if the integrability conditions are satisfied

$$
\partial_{\nu}^{(x)} \partial_{\mu}^{(L)} F=\partial_{\mu}^{(L)} \partial_{\nu}^{(x)} F
$$

This constraints the possible values of the constants $\alpha_{I J}$

$$
\alpha_{P B} \alpha_{B P}=\alpha_{P P} \alpha_{B B}-\alpha_{A A}
$$

which means

$$
\alpha_{A A}=\operatorname{det}(\alpha) \equiv \operatorname{det}\left(\begin{array}{ll}
\alpha_{P P} & \alpha_{P B} \\
\alpha_{B P} & \alpha_{B B}
\end{array}\right) .
$$

Imposing this constraint, the equations (4.10) transforms into the form

$$
\begin{aligned}
& \partial_{\mu}^{(L)} F=\alpha_{P B} \alpha_{B B} L_{\mu}^{\prime}+\alpha_{P B} \alpha_{B P} x_{\mu}^{\prime} \\
& \partial_{\nu}^{(x)} F=\alpha_{B P} \alpha_{P B} L_{\nu}^{\prime}+\alpha_{P P} \alpha_{B P} x_{\nu}^{\prime} .
\end{aligned}
$$

which can be easily integrated (again up to the additive constant corresponding to trivial shift of $\phi$ )

$$
\begin{aligned}
F & =\int_{0}^{\left(x^{\prime}, L^{\prime}\right)}\left(\mathrm{d} x^{\prime} \cdot \partial^{(x)} F+\mathrm{d} L^{\prime} \cdot \partial^{(L)} F\right) \\
& =\frac{1}{2}\left(\alpha_{P B} \alpha_{B B} L^{\prime 2}+2 \alpha_{P B} \alpha_{B P} x^{\prime} \cdot L^{\prime}+\alpha_{P P} \alpha_{B P} x^{\prime 2}\right)
\end{aligned}
$$

As a result we get the most general formulae ${ }^{12}$ for the duality transformation of the coset coordinates in the form

$$
\begin{aligned}
x^{\mu} & =\alpha_{P P} x^{\prime \mu}+\alpha_{P B} L^{\prime \mu}, \quad L^{\mu}=\alpha_{B B} L^{\prime \mu}+\alpha_{B P} x^{\prime \mu} \\
\phi & =\operatorname{det}(\alpha) \phi^{\prime}+\frac{1}{2}\left(\alpha_{P B} \alpha_{B B} L^{\prime 2}+2 \alpha_{P B} \alpha_{B P} x^{\prime} \cdot L^{\prime}+\alpha_{P P} \alpha_{B P} x^{2}\right) .
\end{aligned}
$$

Under this transformation the basic building blocs of the Galileon Lagrangian transform as

$$
\begin{aligned}
& \omega_{B}^{\mu}=\alpha_{B B} \omega_{B}^{\prime \mu}+\alpha_{B P} \omega_{P}^{\prime \mu} \\
& \omega_{P}^{\mu}=\alpha_{P B} \omega_{B}^{\prime \mu}+\alpha_{P P} \omega_{P}^{\prime \mu} \\
& \omega_{A}=\operatorname{det}(\alpha) \omega_{A}^{\prime} .
\end{aligned}
$$

These transformations are parametrized by four constants arranged in the real $2 \times 2$ matrix

$$
\alpha=\left(\begin{array}{ll}
\alpha_{P P} & \alpha_{P B} \\
\alpha_{B P} & \alpha_{B B}
\end{array}\right) .
$$

\footnotetext{
${ }^{12} \mathrm{Up}$ to the remnants of the omitted additive constants, as discussed above.
} 
Imposing the IHC constraint (3.25) we get finally

$$
\begin{aligned}
x & =\alpha_{P P} x^{\prime}+\alpha_{P B} \partial^{\prime} \phi^{\prime} \\
\phi & =\operatorname{det}(\alpha) \phi^{\prime}+\frac{1}{2}\left(\alpha_{P B} \alpha_{B B} \partial^{\prime} \phi^{\prime} \cdot \partial^{\prime} \phi^{\prime}+2 \alpha_{P B} \alpha_{B P} x^{\prime} \cdot \partial^{\prime} \phi^{\prime}+\alpha_{P P} \alpha_{B P} x^{2}\right) \\
\partial \phi & =\alpha_{B B} \partial^{\prime} \phi^{\prime}+\alpha_{B P} x^{\prime} .
\end{aligned}
$$

Let us note that the last formula of (4.19) (the transformation of $\partial \phi$ ) is compatible with the first two as a result of the compatibility of the IHC constraint with the coordinate transformation mentioned above. We can also prove this easily by explicit calculation (see appendix B).

Let us finally write down the explicit formula for the duality in terms of the Galileon action. It is expressed by the identity

$$
S[\phi]=S_{\alpha}\left[\phi^{\prime}\right]
$$

where

$$
\begin{aligned}
S[\phi] & =\int \mathrm{d}^{d} x \sum_{n=1}^{d+1} d_{n} \mathcal{L}_{n} \\
S_{\alpha}[\phi] & =\int \mathrm{d}^{d} x \sum_{n=1}^{d+1} d_{n}(\alpha) \mathcal{L}_{n}
\end{aligned}
$$

and the couplings of the two dual action are interrelated as

$$
d_{n}(\alpha)=\sum_{m=1}^{d+1} A_{n m}(\alpha) d_{m}
$$

where the matrix $A_{n m}(\alpha)$ has the following form

$A_{n m}(\alpha)=\operatorname{det}(\alpha) \frac{m}{n} \sum_{k=0}^{d-m+1} \sum_{l=0}^{m-1}\left(\begin{array}{c}d-m+1 \\ k\end{array}\right)\left(\begin{array}{c}m-1 \\ l\end{array}\right) \alpha_{P B}^{k} \alpha_{B B}^{l} \alpha_{P P}^{d-m+1-k} \alpha_{B P}^{m-1-l} \delta_{n, l+k+1}$.

\section{$5 G L(2, \mathrm{R})$ group of the Galileon dualities}

The duality transformations introduced in the previous section has natural $G L(2, \mathbf{R})$ group structure under compositions. This is immediately seen from their action on the 1-forms $\omega_{A}$, $\omega_{P}^{\mu}$ and $\omega_{B}^{\mu}$ (cf. (4.17)) and on the coset coordinates $x^{\mu}$ and $L^{\mu}$. The duality transformation is in one-to-one correspondence with the matrix

$$
\alpha=\left(\begin{array}{cc}
\alpha_{P P} & \alpha_{P B} \\
\alpha_{B P} & \alpha_{B B}
\end{array}\right)
$$

and composition of two duality transformations corresponding to the matrices $\alpha$ and $\beta$ is again a duality transformation described by matrix $\alpha \cdot \beta$. The condition $\operatorname{det} \alpha \neq 0$ ensures regularity ${ }^{13}$ of the transformation of the coordinates on the coset space (4.16).

\footnotetext{
${ }^{13}$ The Jacobian of the transformation $(4.16)$ is $(\operatorname{det}(\alpha))^{2}$
} 
A little bit less obvious is the group property for the duality transformation of $\phi$. To demonstrate it let us rewrite (4.16) in the form

$$
\begin{aligned}
X & =\alpha \cdot X^{\prime} \\
\phi & =\operatorname{det} \alpha \phi^{\prime}+\frac{1}{2} X^{\prime T} \cdot \widehat{\alpha} \cdot \alpha \cdot X^{\prime}
\end{aligned}
$$

where

$$
X=\left(\begin{array}{c}
x \\
L
\end{array}\right), \quad \widehat{\alpha}=\left(\begin{array}{cc}
\alpha_{B P} & 0 \\
0 & \alpha_{P B}
\end{array}\right)
$$

Then a composition of two dualities means

$$
\begin{aligned}
X & =\alpha \cdot X^{\prime}=\alpha \cdot\left(\beta \cdot X^{\prime \prime}\right)=(\alpha \cdot \beta) \cdot X^{\prime \prime} \\
\phi & =\operatorname{det} \alpha \phi^{\prime}+\frac{1}{2} X^{\prime T} \cdot \widehat{\alpha} \cdot \alpha \cdot X^{\prime} \\
& =\operatorname{det}(\alpha \cdot \beta) \phi^{\prime \prime}+\frac{1}{2} X^{\prime \prime T} \cdot\left((\widehat{\beta} \cdot \beta) \operatorname{det} \alpha+\beta^{T} \cdot \widehat{\alpha} \cdot \alpha \cdot \beta\right) \cdot X^{\prime \prime}
\end{aligned}
$$

However, as can be proved by direct calculation,

$$
(\widehat{\beta} \cdot \beta) \operatorname{det} \alpha+\beta^{T} \cdot \widehat{\alpha} \cdot \alpha \cdot \beta=(\widehat{\alpha \cdot \beta}) \cdot(\alpha \cdot \beta)
$$

and therefore

$$
\phi=\operatorname{det}(\alpha \cdot \beta) \phi^{\prime \prime}+\frac{1}{2} X^{\prime \prime T} \cdot(\widehat{\alpha \cdot \beta}) \cdot(\alpha \cdot \beta) \cdot X^{\prime \prime}
$$

as expected.

On the space $D_{d+1}$ of the Galileon theories, which can be treated as a $d+1$ dimensional real space with elements

$$
\mathbf{d}=\left(\begin{array}{c}
d_{1} \\
d_{2} \\
\vdots \\
d_{d+1}
\end{array}\right),
$$

corresponding to $d+1$-tuples of the couplings $d_{n}$, we have a linear representation of the duality group $G L(2, \mathbf{R})$ by the matrices $A_{n m}(\alpha)$ explicitly given by (4.24).

\subsection{Special cases}

Let us now discuss some important special cases. The duality transformations corresponding to the one-parameter subgroup of matrices

$$
\alpha_{\mathrm{dS}}(\zeta)=\left(\begin{array}{cc}
1 & 0 \\
2 \zeta & 1
\end{array}\right)
$$

which satisfy

$$
\alpha_{\mathrm{dS}}(\zeta) \cdot \alpha_{\mathrm{dS}}\left(\zeta^{\prime}\right)=\alpha_{\mathrm{dS}}\left(\zeta^{\prime}\right) \cdot \alpha_{\mathrm{dS}}(\zeta)=\alpha_{\mathrm{dS}}\left(\zeta+\zeta^{\prime}\right),
$$

result in the following explicit transformation

$$
x=x^{\prime}, \quad \phi=\phi^{\prime}+\zeta x^{2}, \quad \partial \phi=\partial^{\prime} \phi^{\prime}+2 \zeta x^{\prime} .
$$


Fixing the parameter $\zeta=H^{2} / 4$ the dual theory can be interpreted as an expansion of the original Galileon field about the de Sitter solution

$$
\phi_{\mathrm{dS}}=\frac{1}{4} H^{2} x^{2}
$$

The fact, that the fluctuations $\phi^{\prime}$ about such a background are described by a dual Galileon Lagrangian has been established already in the seminal paper [4]. For the transformation of the couplings we get explicitly

$$
d_{n}\left(\alpha_{\mathrm{dS}}(\zeta)\right)=\sum_{m=n}^{d+1}\left(\begin{array}{c}
m \\
n
\end{array}\right)(2 \zeta)^{m-n} d_{m}
$$

Another example concerns the following matrix

$$
\alpha_{L}=\left(\begin{array}{ll}
0 & 1 \\
1 & 0
\end{array}\right)
$$

It results in the duality transformation

$$
x=\partial^{\prime} \phi^{\prime}, \quad \phi=-\phi^{\prime}+x^{\prime} \cdot \partial^{\prime} \phi^{\prime}, \quad \partial \phi=x^{\prime}
$$

which can be rewritten in the more symmetric form as

$$
x \cdot x^{\prime}=\phi(x)+\phi^{\prime}\left(x^{\prime}\right)
$$

and which corresponds to the Legendre transformation. Duality properties of the Galileon theory with respect to this transformation has been discussed in detail in [11]. Explicit form for the dual couplings reads

$$
d_{n}\left(\alpha_{L}\right)=-\frac{d-n+2}{n} d_{d-n+2}
$$

Let us now assume the diagonal matrix

$$
\alpha_{S}(\lambda)=\left(\begin{array}{cc}
\lambda & 0 \\
0 & \lambda^{-\Delta-1}
\end{array}\right)
$$

corresponding to the scaling transformation ( $\Delta$ is the Galileon scaling dimension)

$$
x=\lambda x^{\prime}, \quad \phi=\lambda^{-\Delta} \phi^{\prime}, \quad \partial \phi=\lambda^{-\Delta-1} \partial^{\prime} \phi^{\prime}
$$

for which the dual couplings simply scale according their dimension as

$$
d_{n}\left(\alpha_{S}(\lambda)\right)=\lambda^{d+2-n(\Delta+2)} d_{n}
$$

More general scaling is also possible, namely

$$
\alpha_{S}(\lambda, \kappa)=\left(\begin{array}{ll}
\lambda & 0 \\
0 & \kappa
\end{array}\right)
$$


for which

$$
x=\lambda x^{\prime}, \quad \phi=\lambda \kappa \phi^{\prime}, \quad \partial \phi=\kappa \partial^{\prime} \phi^{\prime}
$$

and in the dual theory

$$
d_{n}\left(\alpha_{S}(\lambda, \kappa)\right)=\kappa^{n} \lambda^{d-n+2} d_{n} .
$$

Let us assume now duality transformations induced by the matrices of the form ${ }^{14}$

$$
\alpha_{D}(\theta)=\left(\begin{array}{cc}
1 & -2 \theta \\
0 & 1
\end{array}\right)
$$

which represents a one-parameter subgroup

$$
\alpha_{D}(\theta) \cdot \alpha_{D}\left(\theta^{\prime}\right)=\alpha_{D}\left(\theta^{\prime}\right) \cdot \alpha_{D}(\theta)=\alpha_{D}\left(\theta+\theta^{\prime}\right) .
$$

The corresponding coordinate and field transformation reads

$$
x=x^{\prime}-2 \theta \partial^{\prime} \phi^{\prime}, \quad \phi=\phi^{\prime}-\theta \partial^{\prime} \phi^{\prime} \cdot \partial^{\prime} \phi^{\prime}, \quad \partial \phi=\partial^{\prime} \phi^{\prime} .
$$

Such a type of duality (with special value of the parameter $\theta$ ) has been discussed in the papers $[17,18,37]$ and its one-parametric group structure has been recognized in a very recent paper [38]. The couplings transform according to

$$
d_{n}\left(\alpha_{D}(\theta)\right)=\frac{1}{n} \sum_{m=1}^{n} m\left(\begin{array}{c}
d-m+1 \\
n-m
\end{array}\right)(-2 \theta)^{n-m} d_{m} .
$$

It is obvious, that any duality transformation can be obtained as a combination of the above elementary types of transformations. Indeed, for general matrix $\alpha$ we can write the following decomposition

$$
\left(\begin{array}{cc}
\alpha_{P P} & \alpha_{P B} \\
\alpha_{B P} & \alpha_{B B}
\end{array}\right)=\left(\begin{array}{cc}
\alpha_{P P} & 0 \\
0 & \alpha_{P P}^{-1} \operatorname{det}(\alpha)
\end{array}\right)\left(\begin{array}{cc}
1 & 0 \\
\alpha_{P P} \alpha_{B P} \operatorname{det}^{-1}(\alpha) & 1
\end{array}\right)\left(\begin{array}{cc}
1 & \alpha_{P P}^{-1} \alpha_{P B} \\
0 & 1
\end{array}\right) .
$$

Let us give another simple example of such a type of decomposition. For instance, we have

$$
\alpha_{D}(\theta)=\left[\alpha_{S}\left(1,(2 \theta)^{-1}\right) \cdot \alpha_{\mathrm{dS}}\left(-2^{-1}\right)\right] \cdot \alpha_{\mathrm{L}} \cdot\left[\alpha_{\mathrm{dS}}\left(2^{-1}\right) \cdot \alpha_{S}(1,-2 \theta)\right],
$$

and therefore we can understand the one-parametric duality (5.25) as a Legendre transformation of the function $\psi^{\prime}\left(x^{\prime}\right)$ into $\psi(x)$ where ${ }^{15}$

$$
\psi^{\prime}\left(x^{\prime}\right)=\frac{1}{2} x^{\prime 2}-2 \theta \phi^{\prime}\left(x^{\prime}\right), \quad \psi(x)=\frac{1}{2} x^{2}+2 \theta \phi(x)
$$

which can be written in the symmetric form (cf. (5.15)) as

$$
x \cdot x^{\prime}=\psi(x)+\psi^{\prime}\left(x^{\prime}\right) .
$$

\footnotetext{
${ }^{14}$ The rationale for the minus sign of the element $\alpha_{P B}$ is that with this choice the infinitesimal form of this duality transformation is

$$
\phi(x)=\phi^{\prime}(x)+\theta \partial \phi^{\prime}(x) \cdot \partial \phi^{\prime}(x) .
$$

See appendix A for bottom up construction of the finite duality transformation from the infinitesimal one.

${ }^{15}$ The function $\psi^{\prime}\left(x^{\prime}\right)$ can be obtained by means of application of the dual transformation corresponding to the product of matrices in the second square brackets in (5.28), similarly for $\psi(x)$.
} 


\subsection{Duality invariants}

As we will see in the following sections, the one parameter subgroup of duality transformations (5.25) is the most interesting one relevant from the point of view of physical applications. Let us briefly comment on some properties of its representation on the Galileon theory space $D_{d+1}$ (see (5.8)). Any classical Galileon Lagrangian $\mathcal{L}=\sum_{n=1}^{d+1} d_{n} \mathcal{L}_{n}$ corresponds to the $d+1$-tuple $\mathbf{d}$ of the couplings $d_{n}$

$$
\mathbf{d}=\left(\begin{array}{c}
d_{1} \\
d_{2} \\
\vdots \\
d_{d+1}
\end{array}\right) \in D_{d+1}
$$

and the one parametric subgroup (5.25) of duality transformation $\alpha_{D}(\theta)$ acts on this tuple linearly via the matrix $\mathbf{A}(\theta) \equiv A_{n m}\left(\alpha_{D}(\theta)\right)$ (see (5.26))

$$
\mathbf{A}(\theta)_{n m}=\frac{1}{n} \sum_{m=1}^{n} m\left(\begin{array}{c}
d-m+1 \\
n-m
\end{array}\right)(-2 \theta)^{n-m} .
$$

As we will see in the next sections, some of the relevant physical quantities (e.g. the $S$ matrix) are invariant with respect to the duality transformations $\alpha_{D}(\theta)$. Such quantities are therefore functions of the invariant combinations of the couplings $d_{n}$. Here we will give a classification of such invariants built from $d_{n}$. The main idea behind this classification is to identify these invariants with conserved integrals of motion of a system of first order differential equations for $\mathbf{d}(\theta)=\mathbf{A}(\theta) \cdot \mathbf{d}$ with $\theta$ taken as the evolution parameter.

First, because the matrices $\mathbf{A}(\theta)$ are lower triangular matrices, any subspace $D_{d+1}^{(k)} \subset$ $D_{d+1}$ spanned by the $d+1$-tuples with first $k$ couplings equal to zero (i.e. $D_{d+1}^{(k)}=\left\{\mathbf{d} \mid d_{n}=0\right.$ for $n \leq k\}$ ) is left invariant by $\mathbf{A}(\theta)$. We can therefore restrict ourselves to some fixed $D_{d+1}^{(k)}$ in what follows. ${ }^{16}$

Note also that $\alpha_{D}(\theta)$ is a one-parametric subgroup and thus the matrices $\mathbf{A}(\theta)$ satisfy a differential equation

$$
\frac{\mathrm{d}}{\mathrm{d} \theta} \mathbf{A}(\theta)=\boldsymbol{T} \cdot \mathbf{A}(\theta)
$$

where

$$
T_{m n}=\left.\frac{\mathrm{d}}{\mathrm{d} \theta} A_{n m}\left(\alpha_{D}(\theta)\right)\right|_{\theta=0}=-2 \frac{n-1}{n}(d-n+2) \delta_{n, m+1} .
$$

Consequently we get for $d+1-k$-tuple $\mathbf{d}(\theta) \equiv d_{n}\left(\alpha_{D}(\theta)\right) \in D_{d+1}^{(k)}$

$$
\frac{\mathrm{d}}{\mathrm{d} \theta} \mathbf{d}(\theta)=\boldsymbol{T} \cdot \mathbf{d}(\theta) .
$$

This is a system of $d-k$ nontrivial ordinary differential equations (note that the first of the equations (5.35) is trivial

$$
\frac{\mathrm{d}}{\mathrm{d} \theta} d_{k+1}(\theta)=0
$$

\footnotetext{
${ }^{16}$ For the physical applications it is natural to set $d_{1}=0$ in order to avoid tadpoles and assume therefore the subspace $D_{d+1}^{(1)}$.
} 
i.e. $d_{k+1}$ can be taken as fixed ${ }^{17}$ once for ever) describing the "running" of the couplings with the change of the duality parameter $\theta$. Of course, the solutions are just $d_{n}\left(\alpha_{D}(\theta)\right)$ given by (5.26) with $d_{n}, n>k$ as the initial conditions at $\theta=0$. Such a system have in general $d-k-1$ functionally independent integrals of motion (we denote them $I_{k+3}, I_{k+4}, \ldots, I_{d+1}$ for reason which will be clear from the construction) which do not depend explicitly on $\theta$. Once these are known, any other such an integral of motion can be then expressed as

$$
I=f\left(I_{k+3}, I_{k+4}, \ldots, I_{d+1}\right),
$$

where $f$ is some function. The set $I_{k+3}, I_{k+4}, \ldots, I_{d+1}$ represents therefore a basis of the $\alpha_{D}(\theta)$ duality invariants on the subspace $D_{d+1}^{(k)}$ of the Galileon theory space.

The set of independent invariants $I_{k+3}, I_{k+4}, \ldots, I_{d+1}$ can be constructed by means of elimination of the initial conditions and $\theta$ the from the solution (5.26). This can be done as follows. Note that (5.26) for $n=k+2$ and $d_{n}=0$ for $n \leq k$ reads

$$
d_{k+2}(\theta)=d_{k+2}-2 \theta \frac{(k+1)(d-k)}{k+2} d_{k+1}
$$

and thus we have unique solution $\theta^{*}$ for $\theta$ such that $d_{k+2}\left(\theta^{*}\right)=0$. According to the group property we can rewrite the solution of (5.35) in the form

$$
\mathbf{d}(\theta)=\mathbf{A}\left(\theta-\theta^{*}\right) \cdot \mathbf{d}\left(\theta^{*}\right)
$$

with new initial conditions $\mathbf{d}\left(\theta^{*}\right)$. Inverting (5.38) we get

$$
\mathbf{d}\left(\theta^{*}\right)=\mathbf{A}\left(\theta^{*}-\theta\right) \cdot \mathbf{d}(\theta)
$$

the right hand side of which is $\theta$ independent. For $d_{k+2}$ the equation (5.38) reads

$$
d_{k+2}(\theta)=-2\left(\theta-\theta^{*}\right) \frac{(k+1)(d-k)}{k+2} d_{k+1}
$$

and thus we can easily eliminate $\theta-\theta^{*}$ solely in terms of $d_{k+2}(\theta)$. Inserting now this for the explicit $\theta-\theta^{*}$ dependence into (5.39) for $n=k+3, \ldots, d+1$ we get the desired integrals of motion $I_{l}\left(d_{k+2}(\theta), \ldots, d_{d+1}(\theta)\right)$. Their interpretation is clear, according to our construction $I_{l}$ represents a value of couplings $d_{l}$ in the theory dual with the original one such that in the dual theory the coupling $d_{k+2}$ is zero. These integrals form the basis of the $\alpha_{D}(\theta)$ duality subgroup invariants on the Galileon theory subspace $D_{d+1}^{(k)}$ we started with.

Let us illustrate this general construction of $\alpha_{D}(\theta)$ duality invariants in the case of three and four dimensional Galileon theory. We will restrict ourselves to the theory subspaces $D_{4}^{(1)}$ and $D_{5}^{(1)}$, i.e. we set in both cases $d_{1}=0$, and we further fix $d_{2}$ for $d=3,4$ as $1 / 4$ and $1 / 12$ respectively. For $d=3$ we get from (5.26)

$$
\begin{aligned}
& d_{3}(\theta)=d_{3}-\frac{2}{3} \theta \\
& d_{4}(\theta)=d_{4}-\frac{3}{2} d_{3} \theta+\frac{1}{2} \theta^{2}
\end{aligned}
$$

\footnotetext{
${ }^{17}$ For instance, for $k=1$ it is natural to set $d_{2}=1 / 12$ in order to normalize the kinetic term of the Galileon as usual.
} 
and according the general recipe, the only $\alpha_{D}(\theta)$ duality invariant is $I_{4}=d_{4}\left(\theta^{*}\right)$ with $\theta^{*}=3 d_{3} / 2$, explicitly

$$
I_{4}=d_{4}-\frac{9}{8} d_{3}^{2} .
$$

For $d=4$ the $\alpha_{D}(\theta)$ duality transformation reads

$$
\begin{aligned}
& d_{3}(\theta)=d_{3}-\frac{1}{3} \theta \\
& d_{4}(\theta)=d_{4}-3 \theta d_{3}+\frac{1}{2} \theta^{2} \\
& d_{5}(\theta)=d_{5}-\frac{8}{5} \theta d_{4}+\frac{12}{5} \theta^{2} d_{3}-\frac{4}{15} \theta^{3},
\end{aligned}
$$

and we have two independent duality invariants $I_{4,5}=d_{4,5}\left(\theta^{*}\right)$ where $\theta^{*}=3 d_{3}$, explicitly

$$
\begin{aligned}
& I_{4}=d_{4}-\frac{9}{2} d_{3}^{2} \\
& I_{5}=d_{5}-\frac{24}{5} d_{3} d_{4}+\frac{72}{5} d_{3}^{3} .
\end{aligned}
$$

\section{Applications}

Two Galileon theories connected by duality are different theories. Therefore all the properties of such theories cannot be the same. However, it does not mean that the dual theories cannot be used to describe the same physical reality. We have only to identify carefully those physical observables that are dual to each other in both theories. Omitting this aspect of the duality might lead to apparent paradoxes. A closely related aspect of the duality is "calculational". Because the duality relates different Galileon theories, its main benefit is based on the possibility to solve a given problem in the simplest exemplar of the set of theories connected by duality. Then the result can be translated back to the apparently more complex original theory for which the problem has been formulated. In order to realize this approach effectively it is necessary to establish the correct interrelation of the observables in both theories. In subsections $6.1,6.2$ and 6.3 we will discuss this issue in more detail. We will show that the classical covariant phase spaces of two theories connected by duality are (at least formally) in one-to-one correspondence. The same is also true for classical observables for which the duality transformation can be established.

Also the (off-shell) symmetries of the Galileon theories are realized differently within the dual theories. Some of them are not directly visible from the form of the classical dual Lagrangian, in this sense they are hidden but still present in the dual theory. In subsection 6.4 we give some elementary examples of such hidden symmetries.

An exceptional role play those physical observables that are invariants of the duality. Only such observables are independent on the choice of the representative in the class of theories connected by duality. As we have mentioned in the previous section, the most useful duality is the one-parametric subgroup $\alpha_{D}(\theta)$, which is (together with $\alpha_{\mathrm{L}}$ ) the only one for which the field and coordinate transformation is nontrivial. Therefore the class of observables invariant with respect to this subgroup are the most important ones. These 
observables give the same result on the whole class of Galileon theories connected by $\alpha_{D}(\theta)$ duality. In subsection 6.5 we will show that the tree level $S$ matrix belongs to the class of $\alpha_{D}(\theta)$ invariant quantities. This will enable us to understand the structure of the results (2.15), (2.16) and (2.17) for the lowest scattering amplitudes (see subsection 6.6). We will also give a simple alternative derivation of these results using their properties under duality. As a next step we will discuss the loop corrections in the Galileon theory in the framework of perturbative low energy effective expansion and the properties of these corrections under duality (see subsections 6.8 and 6.9).

Because the tree level $S$ matrix is invariant of the duality subgroup $\alpha_{D}(\theta)$, it is useful to classify the Galileon theories with respect to this subgroup, i.e. to find all nontrivial classes of the Galileon theories modulo $\alpha_{D}(\theta)$. At the same time we get also classification of all the nontrivial $S$ matrices. In subsection 6.7 we provide such a classification using the duality invariants $I_{k+2}, \ldots, I_{d+1}$ introduced in the previous section in three and four dimensions.

All the above aspects of the duality will be illustrated using several explicit examples both on classical and quantum levels.

In what follows we almost exclusively work in four dimensions with Minkowski metric and in the Galileon Lagrangian we set $d_{1}=0$ to avoid the tadpole and $d_{2}=1 / 12$ to get a canonical normalization of the kinetic term.

\subsection{Classical solutions}

As the calculational aspect of the duality is concerned, in some cases the duality can help us to find solutions of the classical equation of motion very efficiently. Let $\phi_{\alpha}(x)$ be the dual transformation of the field configuration $\phi(x)$ under the $\alpha \in G L(2, \mathbf{R})$ (cf. (4.19)). The definition of the corresponding dual action $S_{\alpha}$ (cf. (4.20))

$$
S_{\alpha}[\phi]=S\left[\phi_{\alpha}\right]
$$

then guarantees that, provided $\phi_{*}(x)$ is a minimum (or stationary point) of the dual action $S_{\alpha}$, the dual configuration $\left(\phi_{*}\right)_{\alpha}(x)$ realizes a minimum (or stationary point) of the original action $S$. In many cases we can choose the matrix $\alpha$ in such a way that we can solve easily the equation of motion for the action $S_{\alpha}$ and find explicitly the dual of this solution simultaneously. This gives us immediately the solution of the apparently much more complicated equation of motion for the original action $S$. Such a method for finding solutions of Galileon equation of motion is usually efficient when we seek after a solution with additional symmetry which effectively reduces the dimensionality of the space-time. It is known that in such a case only limited subset of couplings $d_{n}$ enter the equation of motion [39] and the duality transformation with properly chosen matrix $\alpha$ can further reduce this subset. In the ideal case the dual equation of motion becomes that of the free theory but also in other cases such an approach might be useful.

More formally and in more detail, the functional derivative of (6.1) with respect to $\phi(x)$ gives

$$
\frac{\delta S_{\alpha}[\phi]}{\delta \phi(x)}=\int \mathrm{d}^{d} z \frac{\delta S\left[\phi_{\alpha}\right]}{\delta \phi_{\alpha}(z)} \frac{\delta \phi_{\alpha}(z)}{\delta \phi(x)}
$$


Therefore we get the following relation between the stationary points of both actions

$$
\left.\frac{\delta S\left[\phi_{\alpha}\right]}{\delta \phi_{\alpha}(z)}\right|_{\left(\phi_{*}\right)_{\alpha}}=\left.0 \Longrightarrow \frac{\delta S_{\alpha}[\phi]}{\delta \phi(x)}\right|_{\phi_{*}}=0
$$

and provided the duality transformation induced by the configuration $\phi_{*}$ is invertible, also the reversed implication holds.

The invertibility of the duality transformation is related to the operator $\delta \phi_{\alpha}(z) / \delta \phi(x)$. For further convenience, let us calculate $\delta \phi_{\alpha}(z) / \delta \phi(x)$ explicitly for the case of the subgroup $\alpha_{D}(\theta)$ given by (5.23) and (5.25). Note that the duality transformation under the one parametric subgroup $\alpha_{D}(\theta)$ (here we denote $\phi_{\alpha_{D}(\theta)}$ simply as $\phi_{\theta}$ and analogously for $x_{\theta}$ )

$$
\begin{aligned}
x_{\theta} & =x-2 \theta \partial \phi(x) \\
\phi_{\theta}\left(x_{\theta}\right) & =\phi(x)-\theta \partial \phi(x) \cdot \partial \phi(x)
\end{aligned}
$$

can be rewritten in the inverted form

$$
\begin{aligned}
y & =X[\phi](y)-2 \theta \partial \phi(X[\phi](y)) \\
\phi_{\theta}(y) & =\phi(X[\phi](y))-\theta \partial \phi(X[\phi](y)) \cdot \partial \phi(X[\phi](y)) .
\end{aligned}
$$

Here $X[\phi](y)$ is the inversion of the coordinate transformation defined as

$$
x=X[\phi]\left(x_{\theta}\right),
$$

and we have explicitly shown the functional dependence of this inversion on $\phi$. Then taking this implicit dependence into account we get

$$
\begin{aligned}
\frac{\delta \phi_{\theta}(z)}{\delta \phi(x)}= & \delta^{(d)}(X[\phi](z)-x)+(\partial \phi)(X[\phi](z)) \cdot \frac{\delta X[\phi](z)}{\delta \phi(x)} \\
& -2 \theta \partial \phi(X[\phi](z)) \cdot \partial \delta^{(d)}(X[\phi](z)-x) \\
& -2 \theta \partial \phi(X[\phi](z)) \cdot \partial \partial \phi(X[\phi](z)) \cdot \frac{\delta X[\phi](z)}{\delta \phi(x)} .
\end{aligned}
$$

But taking a functional derivative of the first equation of (6.5) respect to $\phi(x)$ we get

$$
0=\frac{\delta X[\phi](y)}{\delta \phi(x)}-2 \theta \partial \delta^{(d)}(X[\phi](y)-x)-2 \theta \partial \partial \phi(X[\phi](y)) \cdot \frac{\delta X[\phi](y)}{\delta \phi(x)}
$$

and inserting this to $(6.7)$ we get finally ${ }^{18}$

$$
\frac{\delta \phi_{\theta}(z)}{\delta \phi(x)}=\delta^{(d)}(X[\phi](z)-x) .
$$

\footnotetext{
${ }^{18}$ Note, that the substitution $z \rightarrow z_{\theta}$ and the functional derivative with respect to $\phi(x)$ do not commute. Provided we make this replacement in (6.7) after the functional derivative is taken, we get

$$
\left.\left(\frac{\delta \phi_{\theta}(z)}{\delta \phi(x)}\right)\right|_{z \rightarrow z_{\theta}}=\delta^{(d)}\left(X[\phi]\left(z_{\theta}\right)-x\right)=\delta^{(d)}(z-x) .
$$

On the other hand, making this inserting before the functional differentiation, we change the functional dependence of the differentiated functional and the result is different, namely

$$
\frac{\delta}{\delta \phi(x)}\left(\left.\phi_{\theta}(z)\right|_{z \rightarrow z_{\theta}}\right)=\frac{\delta}{\delta \phi(x)}(\phi(z)-\theta \partial \phi(z) \cdot \partial \phi(z))=\delta^{(d)}(z-x)-2 \theta \partial \phi(z) \cdot \partial \delta^{(d)}(z-x) .
$$
}


This result can be used to find a direct relation between equations of motions in both theories. We have (denoting $S_{\alpha_{D}(\theta)} \equiv S_{\theta}$ for simplicity)

$$
\begin{aligned}
\frac{\delta S_{\theta}[\phi]}{\delta \phi(x)} & =\int \mathrm{d}^{d} z \frac{\delta S\left[\phi_{\theta}\right]}{\delta \phi_{\theta}(z)} \delta^{(d)}(X[\phi](z)-x) \\
& =\int \mathrm{d}^{d} z \operatorname{det}\left(\frac{\partial z_{\theta}}{\partial z}\right) \frac{\delta S\left[\phi_{\theta}\right]}{\delta \phi_{\theta}\left(z_{\theta}\right)} \delta^{(d)}(z-x)
\end{aligned}
$$

where we have substituted $z \rightarrow z_{\theta}=z-2 \theta \partial \phi(z)$ and used (6.6) when passing to the second line. Therefore

$$
\frac{\delta S_{\theta}[\phi]}{\delta \phi(x)}=\operatorname{det}\left(\frac{\partial x_{\theta}}{\partial x}\right) \frac{\delta S\left[\phi_{\theta}\right]}{\delta \phi_{\theta}\left(x_{\theta}\right)}
$$

and explicitly (cf. (2.7))

$$
\sum_{n=1}^{d+1} n d_{n}(\theta) \mathcal{L}_{n-1}^{\mathrm{der}}(\partial \partial \phi(x))=\operatorname{det}\left(\frac{\partial x_{\theta}}{\partial x}\right) \sum_{n=1}^{d+1} n d_{n} \mathcal{L}_{n-1}^{\mathrm{der}}\left(\partial_{\theta} \partial_{\theta} \phi_{\theta}\left(x_{\theta}\right)\right)
$$

Let us note, that the above discussion are in fact not restricted to the one parametric subgroup $\alpha_{D}(\theta)$ but holds also for general duality transformation with general $\alpha \in G L(2, \mathbf{R})$ with the obvious replacement $S_{\theta} \rightarrow S_{\alpha}, \phi_{\theta} \rightarrow \phi_{\alpha}, x_{\theta} \rightarrow x_{\alpha}$ (cf. the general formulae (4.19) and (4.21)). The derivation of the functional derivative $\delta \phi_{\alpha}(x) / \delta \phi(y)$ follows the same logic as for $\delta \phi_{\theta}(x) / \delta \phi(y)$ with minor changes caused by the more complicated formulae for $x_{\alpha}$ and $\phi_{\alpha}$. The result is

$$
\frac{\delta \phi_{\alpha}(z)}{\delta \phi(x)}=\operatorname{det} \alpha \delta^{(d)}(X[\phi](z)-x)
$$

where now $X[\phi](z)$ is the inversion of the coordinate transformation $x_{\alpha}=\alpha_{P P} x+\alpha_{P B} \partial \phi(x)$. In the general case the formula (6.11) reads

$$
\frac{\delta S_{\alpha}[\phi]}{\delta \phi(x)}=\operatorname{det} \alpha \operatorname{det}\left(\frac{\partial x_{\alpha}}{\partial x}\right) \frac{\delta S\left[\phi_{\alpha}\right]}{\delta \phi_{\alpha}\left(x_{\alpha}\right)}
$$

In what follows we give two explicit examples of the applicability of the duality with respect to the subgroup $\alpha_{D}(\theta)$ for finding the solutions of the classical equation of motion, namely the static cylindrically symmetric solution and a point-like source.

\subsubsection{Cylindrically symmetric static solution}

As a first example of the calculational efficiency of duality, we will illustrate its application on a simple and analytically solvable case. We will consider a static axial-symmetric solution of Galilean equations with an external source coupled to the Galileon field as

$$
S_{\text {int }}=\int \mathrm{d}^{4} x \phi(x) T(x)
$$

The source $T$ will be represented by an infinite "cosmic string" along the $x_{1}$-axis with linear density $\sigma>0$

$$
T(x)=-\sigma \delta\left(x^{2}\right) \delta\left(x^{3}\right)
$$


Due to the symmetry, the problem is effectively two-dimensional and therefore the quartic and quintic Galileon couplings are irrelevant (their contributions to the classical equation of motion vanish). Exactly this feature is the key ingredient which makes the duality efficient in this case. By appropriate choice of the dual theory we can effectively eliminate also the cubic coupling and solve the dual problem in the framework of free theory.

Note however, that for general external source, the part $S_{\text {int }}$ of the complete action violates duality. Therefore we cannot in general case simply argue that the duality transformation of the classical solution in the original theory is also a solution of the dual theory with the same external source. However, our source term is very special being local and therefore it modifies the equations of motion only on the set of points of zero measure. As we shall explicitly see, for such a source ${ }^{19}$ the duality works, which illustrates the conclusions made in the very recent papers ${ }^{20}[38,40]$.

Let us first consider the general Galileon theory with all the couplings present. Our axial-symmetric ansatz is

$$
\phi(x) \equiv \phi(z \bar{z}),
$$

where we have introduced the complex coordinates $z$ and $\bar{z}$ :

$$
z=x^{2}+\mathrm{i} x^{3}, \quad \bar{z}=x^{2}-\mathrm{i} x^{3}
$$

i.e.

$$
\partial_{2}=\partial+\bar{\partial}, \quad \partial_{3}=\mathrm{i} \partial-\mathrm{i} \bar{\partial}, \quad \mathrm{d}^{2} z \equiv-\mathrm{i} \mathrm{d} \bar{z} \mathrm{~d} z=2 \mathrm{~d} x^{2} \mathrm{~d} x^{3}
$$

In order to obtain the explicit form for the classical equations of motion we will start with the following useful formula [11]

$$
\mathcal{L}_{4}^{\text {der }}[\eta+w \partial \partial \phi]=4 ! \operatorname{det}[\eta+w \partial \partial \phi]=\sum_{k=0}^{4} w^{k}\left(\begin{array}{l}
4 \\
k
\end{array}\right) \mathcal{L}_{k}^{\operatorname{der}}[\partial \partial \phi],
$$

where we can easily work out the left hand side because the matrix $\eta+w \partial \partial \phi$ is blockdiagonal,

$$
\operatorname{det}[\eta+w \partial \partial \phi]=-1+4 w \partial \bar{\partial} \phi+4 w^{2}\left[\partial^{2} \phi \bar{\partial}^{2} \phi-(\partial \bar{\partial} \phi)^{2}\right] .
$$

Comparing this with the right hand side of (6.20) we get

$$
\begin{aligned}
\mathcal{L}_{1}^{\text {der }}[\partial \partial \phi] & =24 \partial \bar{\partial} \phi \\
\mathcal{L}_{2}^{\text {der }}[\partial \partial \phi] & =16\left[\partial^{2} \phi \bar{\partial}^{2} \phi-(\partial \bar{\partial} \phi)^{2}\right] \\
\mathcal{L}_{3}^{\text {der }}[\partial \partial \phi] & =\mathcal{L}_{4}^{\text {der }}[\partial \partial \phi]=0
\end{aligned}
$$

and therefore the equation of motion with an external source $T$ is

$$
\frac{\delta S}{\delta \phi}=\sum_{n} n d_{n} \mathcal{L}_{n-1}^{\text {der }}+T=2 d_{2} \mathcal{L}_{1}^{\text {der }}[\partial \partial \phi]+3 d_{3} \mathcal{L}_{2}^{\text {der }}[\partial \partial \phi]+T=0
$$

\footnotetext{
${ }^{19}$ This remains true also for the point-like source studied in the next subsection.

${ }^{20}$ See also [18] for discussions of point-like sources.
} 
where we will set $d_{2}=1 / 12$ in the following. In our case $T=-2 \sigma \delta^{(2)}(z, \bar{z})$ so the equation of motion becomes

$$
4 \partial \bar{\partial} \phi+48 d_{3}\left[\partial^{2} \phi \bar{\partial}^{2} \phi-(\partial \bar{\partial} \phi)^{2}\right]=2 \sigma \delta^{(2)}(z, \bar{z})
$$

First we can easily solve the theory for $d_{3}=0$. The axial symmetric solution is (up to a constant term)

$$
\phi(z \bar{z})=\frac{\sigma}{4 \pi} \ln z \bar{z}
$$

In the case when $d_{3} \neq 0$ we can first rewrite the equation of motion to

$$
\frac{1}{z} \bar{\partial}\left[z \bar{z} \phi^{\prime}(z \bar{z})-12 d_{3} z \bar{z} \phi^{\prime}(z \bar{z})^{2}\right]=\frac{\sigma}{2} \sigma \delta^{(2)}(z, \bar{z}),
$$

where the prime means a derivative with respect to $z \bar{z}$. By further integration over the disc with $z \bar{z} \leq R^{2}$ and using the Gauss theorem in two dimension we will arrive to ${ }^{21}$

$$
\phi^{\prime}\left(R^{2}\right)-12 d_{3} \phi^{\prime}\left(R^{2}\right)^{2}=\frac{\sigma}{4 \pi R^{2}},
$$

which can be algebraically solved to

$$
\phi_{ \pm}^{\prime}\left(R^{2}\right)=\frac{1 \pm \sqrt{1-12 \frac{d_{3} \sigma}{\pi R^{2}}}}{24 d_{3}} .
$$

The final result can be obtained by elementary integration. We have two solutions, which is for $d_{3}>0$ defined only for $R^{2}>12 d_{3} \sigma / \pi$ (for $R^{2}<12 d_{3} \sigma / \pi$ this solution has an imaginary part). ${ }^{22}$ We will show in the following how this can be obtained using duality in a much simpler and pure algebraical way.

The transformation of duality under the subgroup $\alpha_{D}(\theta)$ can be expressed in our coordinates as

$$
\begin{aligned}
z_{\theta} & =z+4 \theta \bar{\partial} \phi(z, \bar{z}) \\
\bar{z}_{\theta} & =\bar{z}+4 \theta \partial \phi(z, \bar{z}) \\
\phi_{\theta}\left(z_{\theta}, \bar{z}_{\theta}\right) & =\phi(z, \bar{z})+4 \theta \bar{\partial} \phi(z, \bar{z}) \partial \phi(z, \bar{z})
\end{aligned}
$$

while the remaining coordinates $x^{0}$ and $x^{1}$ are left unchanged (cf. (5.25)). Let us assume that $\phi(z, \bar{z})$ is the solution of the theory (6.25) with $d_{3}=0$. The duality transformation of

\footnotetext{
${ }^{21}$ This is in fact an expected result. As we have mentioned above, the problem is effectively twodimensional and therefore posses two-dimensional spherical symmetry. In any dimension the spherically symmetric Galileon equation reduces to algebraic equation for the first derivative of the field.

${ }^{22}$ For $d_{3}<0$ the solution $\phi_{-}$exhibits the Vainshtein mechanism [5] with Vainshtein radius $R_{V}^{2}=$ $-12 d_{3} \sigma / \pi$. Indeed, outside and inside the Vainshtein radius we have

$$
\frac{\mathrm{d}}{\mathrm{d} R} \phi_{-}= \begin{cases}\frac{\sigma}{2 \pi R}+O\left(R^{-3}\right), & \text { for } R>R_{V} \\ -\left(-\frac{\sigma}{12 d_{3} \pi}\right)^{1 / 2}+O(R), & \text { for } R<R_{V}\end{cases}
$$
}


$\phi(z, \bar{z})$ is then given implicitly as

$$
\begin{aligned}
z_{\theta} & =z+\frac{\sigma \theta}{\pi \bar{z}} \\
\bar{z}_{\theta} & =\bar{z}+\frac{\sigma \theta}{\pi z} \\
\phi_{\theta}\left(z_{\theta}, \bar{z}_{\theta}\right) & =\frac{\sigma}{4 \pi}\left(\ln z \bar{z}+\frac{\sigma \theta}{\pi z \bar{z}}\right) .
\end{aligned}
$$

We have therefore

$$
z_{\theta} \bar{z}_{\theta}=z \bar{z}+\left(\frac{\sigma \theta}{\pi}\right)^{2} \frac{1}{z \bar{z}}+2 \frac{\sigma \theta}{\pi} .
$$

Let us note that for $\theta>0$ the transformation $z \rightarrow z_{\theta}$ double covers the complement of a circle $z_{\theta} \bar{z}_{\theta}<4 \frac{\sigma \theta}{\pi}$; inside of this circle $\phi_{\theta}$ is not defined. For $\theta<0$ this transformation double covers the whole complex plane, the circle $z \bar{z}=-\frac{\sigma \theta}{\pi}$ is mapped to the point $z_{\theta}=0$. The inversion of (6.31) which shall be inserted to the right hand side of $\phi_{\theta}\left(z_{\theta}, \bar{z}_{\theta}\right)$ is then

$$
z \bar{z}=\frac{1}{2}\left(z_{\theta} \bar{z}_{\theta}-2 \frac{\sigma \theta}{\pi} \pm \sqrt{z_{\theta} \bar{z}_{\theta}\left(z_{\theta} \bar{z}_{\theta}-4 \frac{\sigma \theta}{\pi}\right)}\right)
$$

Now the duality means that

$$
S\left[\phi_{\theta}\right]=S_{\theta}[\phi]
$$

where $S$ and $S_{\theta}$ are the actions (without the external source term $S_{\text {int }}$ ) of the general Galileon theory and its $\alpha_{D}(\theta)$ dual respectively. In our case we take the former to be the general interacting theory (with $d_{3} \neq 0$ ) and the latter we identify with its dual chosen in such a way that $d_{3}(\theta)=0$. As we know from (5.43) such a theory can be obtained from the general one by duality transformation with $\theta=3 d_{3}$ and thus for this value the eq. (6.30) is expected to represent the wanted solution of (6.24), (6.27). Let us now verify that it is indeed the case.

Using the duality transformation of the derivatives (cf. the last equation of (5.25))

$$
\partial \phi(z \bar{z})=\bar{z} \phi^{\prime}(z \bar{z})=\partial_{\theta} \phi_{\theta}\left(z_{\theta} \bar{z}_{\theta}\right)=\bar{z}_{\theta} \phi_{\theta}^{\prime}\left(z_{\theta} \bar{z}_{\theta}\right)
$$

we obtain

$$
\phi_{\theta}^{\prime}\left(z_{\theta} \bar{z}_{\theta}\right)=\frac{z}{z_{\theta}} \phi^{\prime}(z \bar{z})=\frac{\bar{z}}{\bar{z}_{\theta}} \phi^{\prime}(z \bar{z})=\left(1+\frac{\sigma \theta}{\pi z \bar{z}}\right)^{-1} \phi^{\prime}(z \bar{z})
$$

Inserting this in the left hand side of (6.27) we get

$$
\begin{aligned}
\phi_{\theta}^{\prime}\left(z_{\theta} \bar{z}_{\theta}\right)-12 d_{3} \phi_{\theta}^{\prime}\left(z_{\theta} \bar{z}_{\theta}\right)^{2} & =\left(1+\frac{\sigma \theta}{\pi z \bar{z}}\right)^{-1} \phi^{\prime}(z \bar{z})-12 d_{3}\left[\left(1+\frac{\sigma \theta}{\pi z \bar{z}}\right)^{-1} \phi^{\prime}(z \bar{z})\right]^{2} \\
& =\frac{\sigma}{4 \pi z \bar{z}}\left(1+\frac{\sigma \theta}{\pi z \bar{z}}\right)^{-2}\left[1+\frac{\sigma\left(\theta-3 d_{3}\right)}{\pi z \bar{z}}\right]
\end{aligned}
$$

where in the last line we used the explicit form of $\phi(z \bar{z})$. Therefore for $\theta=3 d_{3}$ and expressing back $z \bar{z}$ in terms of $z_{\theta} \bar{z}_{\theta}$ we get

$$
\phi_{\theta}^{\prime}\left(z_{\theta} \bar{z}_{\theta}\right)-4 \theta \phi_{\theta}^{\prime}\left(z_{\theta} \bar{z}_{\theta}\right)^{2}=\frac{\sigma}{4 \pi z \bar{z}}\left(1+\frac{\sigma \theta}{\pi z \bar{z}}\right)^{-2}=\frac{\sigma}{4 \pi z_{\theta} \bar{z}_{\theta}}
$$

which means that $\phi_{3 d_{3}}$ is a solution of the equation (6.27). 


\subsubsection{Point-like source}

As a next example, let us repeat the above strategy for the spherically symmetric solution of the galileon equation of motion with point-like source $T(x)=-4 \pi M \delta^{(3)}(\mathbf{x})$. Though the duality does not help us much in solving the most general equation of motion, as we will see, it might be useful in some special cases.

Due to the spherical symmetry the situation is similar to the previous subsection. The problem is effectively three-dimensional and the quintic Galileon coupling thus disappear from the problem. The ansatz for the solution is

$$
\phi(x) \equiv \phi(r)
$$

where $r=|\mathbf{x}|=\sqrt{x^{i} x^{i}}$. After some algebra we end up with the equation of motion in the form (cf. [4] for more details)

$$
\frac{12 d_{2}}{r^{2}}\left(r^{2} \phi^{\prime}(r)\right)^{\prime}-\frac{12 d_{3}}{r^{2}}\left(r \phi^{\prime}(r)^{2}\right)^{\prime}+\frac{8 d_{4}}{r^{2}}\left(\phi^{\prime}(r)^{3}\right)^{\prime}-4 \pi M \delta^{(3)}(\mathbf{x})=0 .
$$

Integrating over $\mathrm{d}^{3} \mathrm{x}=4 \pi r^{2} \mathrm{~d} r$ and assuming canonically normalized kinetic term $\left(d_{2}=\right.$ $1 / 12$ ) we get

$$
r^{2} \phi^{\prime}(r)-12 d_{3} r \phi^{\prime}(r)^{2}+8 d_{4} \phi^{\prime}(r)^{3}=M
$$

which is an algebraic equation for $\phi^{\prime}(r) / r$. The duality transformation of the spherically symmetric static solution $\phi(r)$ reads

$$
\begin{aligned}
x_{\theta}^{0} & =x^{0}, \quad x_{\theta}^{i}=\left(1+2 \theta \frac{\phi^{\prime}(r)}{r}\right) x^{i} \\
\phi_{\theta}\left(x_{\theta}\right) & =\phi(r)+\theta \phi^{\prime}(r)^{2}
\end{aligned}
$$

Therefore, provided $1+2 \theta \phi^{\prime}(r) / r>0$

$$
r_{\theta}=\left(1+2 \theta \frac{\phi^{\prime}(r)}{r}\right) r
$$

and thus $\phi_{\theta}\left(x_{\theta}\right)$ is function of $r_{\theta}$ only. From the general formula $(\partial \phi)_{\theta}\left(x_{\theta}\right)=\partial \phi(x)$ we get further

$$
\phi_{\theta}^{\prime}\left(r_{\theta}\right)=\phi^{\prime}(r)
$$

Now let $\phi$ be a solution of (6.40) with $d_{i} \rightarrow d_{i}(\theta)$, i.e. let

$$
r^{2} \phi^{\prime}(r)-12 d_{3}(\theta) r \phi^{\prime}(r)^{2}+8 d_{4}(\theta) \phi^{\prime}(r)^{3}=M
$$

It is then easy to show, that $\phi_{\theta}\left(r_{\theta}\right)$ is a solution of (6.40). Indeed

$$
\begin{aligned}
& r_{\theta}^{2} \phi_{\theta}^{\prime}\left(r_{\theta}\right)-12 d_{3} r_{\theta} \phi_{\theta}^{\prime}\left(r_{\theta}\right)+8 d_{4}^{3} \phi_{\theta}^{\prime}\left(r_{\theta}\right) \\
= & r^{2}\left(1+2 \theta \frac{\phi^{\prime}(r)}{r}\right)^{2} \phi^{\prime}(r)-12 d_{3} r\left(1+2 \theta \frac{\phi^{\prime}(r)}{r}\right) \phi^{\prime}(r)^{2}+8 d_{4} \phi^{\prime}(r)^{3} \\
= & r^{2} \phi^{\prime}(r)-12\left(d_{3}-\frac{1}{3} \theta\right) r \phi^{\prime}(r)^{2}+8\left(d_{4}-3 d_{3} \theta+\frac{1}{2} \theta^{2}\right) \phi^{\prime}(r)^{3} \\
= & r^{2} \phi^{\prime}(r)-12 d_{3}(\theta) r \phi^{\prime}(r)^{2}+8 d_{4}(\theta) \phi^{\prime}(r)^{3}=M .
\end{aligned}
$$


For $\theta=3 d_{3}$ we can eliminate the cubic Galileon coupling and the equation (6.45) becomes

$$
r^{2} \phi^{\prime}(r)+8 I_{4} \phi^{\prime}(r)^{3}=M,
$$

where $I_{4}$ is the invariant $(5.44)$

$$
I_{4}=\left(d_{4}-\frac{9}{2} d_{3}^{2}\right) .
$$

Moreover, for special case $I_{4}=0$ we can find the solution of (6.46) simply (up to an additive constant) as

$$
\phi(r)=-\frac{M}{r} .
$$

Its dual given by

$$
\begin{aligned}
r_{\theta} & =r\left(1+2 \theta \frac{M}{r^{3}}\right) \\
\phi_{\theta}\left(r_{\theta}\right) & =-\frac{M}{r}+\theta \frac{M^{2}}{r^{4}}=-\frac{M}{r}\left(1-\theta \frac{M}{r^{3}}\right),
\end{aligned}
$$

is for $\theta=3 d_{3}$ a solution of equation

$$
r^{2} \phi^{\prime}(r)-12 d_{3} r \phi^{\prime}(r)^{2}+36 d_{3}^{2} \phi^{\prime}(r)^{3}=M,
$$

which corresponds to two-parametric set of Galileon theories with parameters $d_{3}, d_{5}$ with special quartic coupling $d_{4}=9 d_{3}^{2} / 2$.

Let us assume now a complementary application of duality. For $I_{4}<0$ we can choose

$$
\theta_{ \pm}=3 d_{3} \pm \sqrt{-2 I_{4}},
$$

and eliminate the quartic couplings in the dual action $S_{\theta_{ \pm}}[\phi]$. The dual equation is then

$$
r^{2} \phi^{\prime}(r)-12 d_{3}\left(\theta_{ \pm}\right) r \phi^{\prime}(r)^{2}=M
$$

where

$$
d_{3}\left(\theta_{ \pm}\right)=\mp \frac{1}{3} \sqrt{-2 I_{4}} .
$$

For further convenience let us choose $\theta_{+}$to ensure $d_{3}\left(\theta_{+}\right)<0$. The solution for the derivative is then simply

$$
\phi_{ \pm}^{\prime}(r)=r \frac{1 \pm \sqrt{1-48 d_{3}\left(\theta_{+}\right) \frac{M}{r^{3}}}}{24 d_{3}\left(\theta_{+}\right)}
$$

and integration gives (cf. also [11])

$$
\phi_{ \pm}(r)=\phi_{ \pm}(0)+\frac{r^{2}}{48 d_{3}\left(\theta_{+}\right)} \pm\left(-\frac{M r}{3 d_{3}\left(\theta_{+}\right)}\right)^{1 / 2}{ }_{2} F_{1}\left(-\frac{1}{2}, \frac{1}{6}, \frac{7}{6} ; \frac{r^{3}}{48 d_{3}\left(\theta_{+}\right) M}\right)
$$

According to (6.43), (6.44) we get for the dual transformation of this solution

$$
r_{\theta_{+}}=r\left(1+\theta_{+} \frac{1 \pm \sqrt{1-48 d_{3}\left(\theta_{+}\right) \frac{M}{r^{3}}}}{12 d_{3}\left(\theta_{+}\right)}\right)
$$




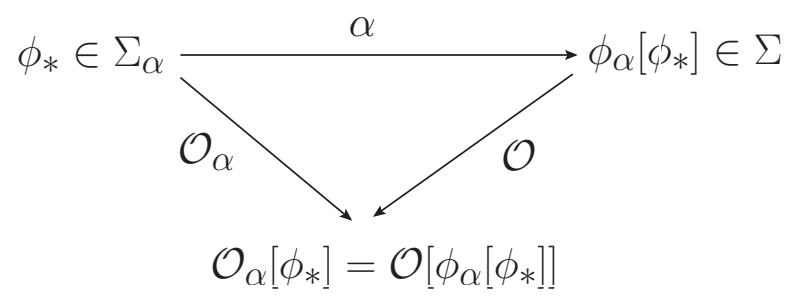

Figure 2. Graphical description of the definition of dual observable. To get the value of the observable $\mathcal{O}$ we can either use the phase space $\Sigma$ of the original theory or the dual phase space $\Sigma_{\alpha}$ and dual observable $\mathcal{O}_{\alpha}$.

$$
\begin{aligned}
\phi_{\theta_{+}}\left(r_{\theta_{+}}\right)_{ \pm}= & \phi_{ \pm}(0)+\frac{r^{2}}{48 d_{3}\left(\theta_{+}\right)} \pm\left(-\frac{M r}{3 d_{3}\left(\theta_{+}\right)}\right)^{1 / 2}{ }_{2} F_{1}\left(-\frac{1}{2}, \frac{1}{6}, \frac{7}{6} ; \frac{r^{3}}{48 d_{3}\left(\theta_{+}\right) M}\right) \\
& +\theta_{+} r^{2}\left(\frac{1 \pm \sqrt{1-48 d_{3}\left(\theta_{+}\right) \frac{M}{r^{3}}}}{24 d_{3}\left(\theta_{+}\right)}\right)^{2}
\end{aligned}
$$

which solves (6.40).

\subsection{Duality of classical observables}

As we have seen in the previous subsection, the most general duality transformation which corresponds to the matrix $\alpha \in G L(2, \mathbf{R})$ assigns to each field configuration $\phi(x)$ its dual configuration $\phi_{\alpha}[\phi](x)$, which is given by the implicit formulae (4.19). With help of this transformation we can define a dual action $S_{\alpha}[\phi]$ to the original action $S[\phi]$ according to the prescription

$$
S_{\alpha}[\phi]=S\left[\phi_{\alpha}[\phi]\right] .
$$

We have shown that provided $\phi_{*}$ is solution of the equation of motion corresponding to the dual action $S_{\alpha}[\phi]$ the dual configuration $\phi_{\alpha}\left[\phi_{*}\right]$ is a solution of the equation of motion for the original action $S[\phi]$. This can be interpreted that there is (at least formally) a correspondence between the (classical) covariant phase spaces $\Sigma$ and $\Sigma_{\alpha}$ of both theories, which is mediated by duality. By covariant phase space we mean the space of all solution of the classical equation of motion i.e. without any constraints on the initial or final data (see [41] for more details), that means the following sets ${ }^{23}$

$$
\Sigma=\left\{\phi_{*}(x) \mid \frac{\delta S\left[\phi_{*}\right]}{\delta \phi(x)}=0\right\}, \quad \Sigma_{\alpha}=\left\{\phi_{*}(x) \mid \frac{\delta S_{\alpha}\left[\phi_{*}\right]}{\delta \phi(x)}=0\right\} .
$$

The correspondence of the covariant phase spaces is then a mapping $\Sigma_{\alpha} \rightarrow \Sigma$ according to the prescription $\phi_{*} \in \Sigma_{\alpha} \rightarrow \phi_{\alpha}\left[\phi_{*}\right] \in \Sigma$.

The physical observables are then real functionals on the covariant phase space, i.e. the mappings $\Sigma \rightarrow \mathbf{R}$ (or $\Sigma_{\alpha} \rightarrow \mathbf{R}$ ). They can be understood as a restriction of the general functionals $\mathcal{O}[\phi]$, defined on all admissible field configurations $\phi$, to the space $\Sigma$ or $\Sigma_{\alpha}$.

\footnotetext{
${ }^{23}$ The covariant phase space can be equipped by symplectic structure e.g. by Peierls brackets.
} 
Two such general functionals $\mathcal{O}$ and $\mathcal{O}^{\prime}$ define then the same observable on the space $\Sigma$ provided their difference vanishes on $\Sigma$, i.e.

$$
\mathcal{O}\left[\phi_{*}\right]-\mathcal{O}^{\prime}\left[\phi_{*}\right]=0 \quad \text { for } \quad \phi_{*} \in \Sigma .
$$

We can enlarge the duality transformation $\phi \rightarrow \phi_{\alpha}[\phi]$ to observables according to the prescription

$$
\mathcal{O}_{\alpha}[\phi]=\mathcal{O}\left[\phi_{\alpha}[\phi]\right]
$$

(see figure 2) where the functional $\mathcal{O}[\phi]$ defines observable on $\Sigma$ and $\mathcal{O}_{\alpha}[\phi]$ defines ${ }^{24}$ the corresponding dual observable on $\Sigma_{\alpha}$. We can therefore freely calculate the value of given "abstract" observable either within the dual theory using the point $\phi_{*} \in \Sigma_{\alpha}$ or within the original theory using the dual point $\phi_{\alpha}\left[\phi_{*}\right] \in \Sigma$. However, we have to take care to use corresponding observables $\mathcal{O}_{\alpha}$ or $\mathcal{O}$ within the dual and original theories respectively.

The above identification of the phase spaces and the corresponding algebras of observables can be used in practical calculations. Note however, that in some cases, a conservation of complexity might take place. Sometimes we end up with dual theory the action $S_{\alpha}$ of which is much simpler than the original one. However, to get a concrete value of some simple observable $\mathcal{O}$ on $\Sigma$ in terms of the simpler dual theory, we have to use much more complicated observable $\mathcal{O}_{\alpha}$ on $\Sigma_{\alpha}$.

\subsection{Fluctuations of classical solutions}

The duality can also be helpful when the small perturbations $\chi(x)$ of solution $\phi_{*}(x)$ of the classical equation of motion are investigated, i.e. when we set $\phi=\phi_{*}+\chi$ in the Galileon action. In this section we will discuss how the duality transformation acts on the field $\chi(x)$ in the linearized theory of fluctuations. We will show that the solution of the linearized fluctuation equation of motion in the dual theory is related by appropriate duality transformation to the corresponding solution within the original theory. This means that the covariant phase spaces and observables in these theories are related by duality. We will also discuss the possible superluminal propagation of the fluctuations in the theories connected by duality and argue that apparent paradoxes (i.e. when healthy theory with (sub)luminal propagation of the fluctuation is dual to superluminally propagating one) stem from the inadequate identification of the dual observables. We will illustrate this issue on explicitly solvable examples.

To start with, let us insert $\phi=\phi_{*}+\chi$ into the Galileon action

$$
S\left[\phi_{*}+\chi\right]=S\left[\phi_{*}\right]+\left.\frac{1}{2} \int \mathrm{d}^{d} x \mathrm{~d}^{d} y \chi(x) \frac{\delta^{2} S[\phi]}{\delta \phi(x) \delta \phi(y)}\right|_{\phi_{*}} \chi(y)+O\left(\chi^{3}\right) .
$$

Here we used the equation of motion for $\phi_{*}$ to eliminate the term linear in $\chi$. The second variation of the Galileon action (i.e. the fluctuation operator) is a local second order

\footnotetext{
${ }^{24}$ Note that this definition is consistent. Indeed, provided the functional $\mathcal{G}[\phi]$ defines the same observable as $\mathcal{F}[\phi]$ (i.e. (6.61) is satisfied) then for $\phi_{*} \in \Sigma_{\alpha}$ and $\phi_{\alpha}\left[\phi_{*}\right]=\left(\phi_{*}\right)_{\alpha} \in \Sigma($ cf. (6.3)). Therefore

$$
\mathcal{F}_{\alpha}\left[\phi_{*}\right]-\mathcal{G}_{\alpha}\left[\phi_{*}\right]=\mathcal{F}\left[\phi_{\alpha}\left[\phi_{*}\right]\right]-\mathcal{G}\left[\phi_{\alpha}\left[\phi_{*}\right]\right]=0 .
$$

As a result, the functionals $\mathcal{F}_{\alpha}[\phi]$ and $\mathcal{G}_{\alpha}[\phi]$ define the same observable on $\Sigma_{\alpha}$.
} 
differential operator of the form

$$
\left.\frac{\delta^{2} S[\phi]}{\delta \phi(x) \delta \phi(y)}\right|_{\phi_{*}}=-g\left[\phi_{*}\right]^{\mu \nu} \partial_{\mu} \partial_{\nu} \delta^{(d)}(x-y)
$$

where (cf. (2.7))

$$
g\left[\phi_{*}\right]^{\mu \nu}=-\sum_{n=2}^{d+1} n(n-1) d_{n} \varepsilon^{\mu \mu_{2} \ldots \mu_{d}} \varepsilon^{\nu \nu_{2} \ldots \nu_{d}} \prod_{i=2}^{n-1} \partial_{\mu_{i}} \partial_{\nu_{i}} \phi_{*}(x) \prod_{j=n}^{d} \eta_{\mu_{j} \nu_{j}} .
$$

Note that the Minkowski tensor $g\left[\phi_{*}\right]^{\mu \nu}$ obeys the following relation

$$
\partial_{\mu} g\left[\phi_{*}\right]^{\mu \nu}=\partial_{\nu} g\left[\phi_{*}\right]^{\mu \nu}=0 .
$$

This enables us to rewrite the quadratic part of the action (6.64) equations of motion for $d>2$ in a form

$$
S\left[\phi_{*}+\chi\right]=S\left[\phi_{*}\right]+\frac{1}{2} \int \mathrm{d}^{d} x \sqrt{\left|G\left[\phi_{*}\right]\right|} G^{\mu \nu}\left[\phi_{*}\right] \partial_{\mu} \chi \partial_{\nu} \chi+O\left(\chi^{3}\right)
$$

where the effective metric $G^{\mu \nu}\left[\phi_{*}\right]$ is given in terms of $g\left[\phi_{*}\right]^{\mu \nu}$ as

$$
G^{\mu \nu}\left[\phi_{*}\right]=\left|\operatorname{det}\left(g\left[\phi_{*}\right]^{\cdot *}\right)\right|^{\frac{1}{2-d}} g^{\mu \nu}\left[\phi_{*}\right]
$$

and $G\left[\phi_{*}\right]=\operatorname{det}\left(G_{\mu \nu}^{-1}\left[\phi_{*}\right]\right)$ with $G_{\mu \sigma}^{-1}\left[\phi_{*}\right] G^{\sigma \nu}\left[\phi_{*}\right]=\delta_{\mu}^{\nu}$. The second term on the right hand side of (6.68) is manifestly invariant with respect to general coordinate transformations $x \rightarrow x^{\prime}$ under which

$$
\chi^{\prime}\left(x^{\prime}\right)=\chi(x), \quad G^{\mu \nu}\left[\phi_{*}\right]^{\prime}\left(x^{\prime}\right)=\partial_{\rho} x^{\prime \mu} \partial_{\sigma} x^{\prime \nu} G^{\rho \sigma}\left[\phi_{*}\right](x)
$$

(i.e. we assume $\chi(x)$ to be a scalar with respect to the diffeomorphisms). The linearized equation of motion for $\chi(x)$ then reads

$$
\left.\int \mathrm{d}^{d} y \frac{\delta^{2} S[\phi]}{\delta \phi(x) \delta \phi(y)}\right|_{\phi_{*}} \chi(y)=-g\left[\phi_{*}\right]^{\mu \nu}(x) \partial_{\mu} \partial_{\nu} \chi(x)=0 .
$$

or in manifestly invariant form ${ }^{25}$

$$
\chi_{; \mu}^{; \mu}=\frac{1}{\sqrt{\left|G\left[\phi_{*}\right]\right|}} \partial_{\mu}\left(\sqrt{\left|G\left[\phi_{*}\right]\right|} G^{\mu \nu}\left[\phi_{*}\right] \partial_{\nu} \chi\right)=0
$$

The fluctuations of the classical background $\phi_{*}$ propagate therefore according to the massless Klein-Gordon equation in an effective spacetime with a metric

$$
\mathrm{d} s^{2}=G_{\mu \nu}^{-1}\left[\phi_{*}\right] \mathrm{d} x^{\mu} \mathrm{d} x^{\nu} .
$$

The situation is completely analogous to the case of the small perturbation of the k-essence in a given classical background which has been discussed in detail in [42]. As explained

\footnotetext{
${ }^{25}$ Here ";" means the covariant derivative with respect to the effective metric $G_{\mu \nu}^{-1}\left[\phi_{*}\right]$.
} 
there, the effective metric $G_{\mu \nu}^{-1}\left[\phi_{*}\right]$ defines the cone of influence of the fluctuations $\chi(x)$ by the equation ${ }^{26}$

$$
G_{\mu \nu}^{-1}\left[\phi_{*}\right] N^{\mu} N^{\nu}=0 .
$$

Provided this influence cone is larger than the Minkowski one, i.e. when $N^{2}<0$, the small fluctuations can propagate superluminally.

As we will show in what follows, we can relate the perturbation in original and dual theory through simple duality transformation induced by the background solution $\phi_{*}(x)$. This will enable us to translate the solutions for the linearized equations of motion for perturbations from original to dual theory and vice versa, and to study the effects of the propagation of the perturbations in both theories. For simplicity we will restrict our discussion to the one parameter subgroup $\alpha_{D}(\theta)$, however, it can be easily modified for the general case.

The transformation formula can be formally obtained as follows. Taking the second functional derivative of (6.1) we get

$$
\begin{aligned}
\frac{\delta^{2} S_{\theta}[\phi]}{\delta \phi(x) \delta \phi(y)}= & \int \mathrm{d}^{d} z \mathrm{~d}^{d} w \frac{\delta^{2} S\left[\phi_{\theta}\right]}{\delta \phi_{\theta}(z) \delta \phi_{\theta}(w)} \frac{\delta \phi_{\theta}(z)}{\delta \phi(x)} \frac{\delta \phi_{\theta}(w)}{\delta \phi(y)} \\
& +\int \mathrm{d}^{d} z \frac{\delta S\left[\phi_{\theta}\right]}{\delta \phi_{\theta}(z)} \frac{\delta^{2} \phi_{\theta}(z)}{\delta \phi(x) \delta \phi(y)}
\end{aligned}
$$

Inserting now $\phi \rightarrow \phi_{*}$ the solution of the equation of motion for the action $S_{\theta}$, the second term on the right hand side drops out ${ }^{27}$ (cf. (6.11)) and we have

$$
\left.\frac{\delta^{2} S_{\theta}[\phi]}{\delta \phi(x) \delta \phi(y)}\right|_{\phi_{*}}=\left.\int \mathrm{d}^{d} z \mathrm{~d}^{d} w \frac{\delta \phi_{\theta}(z)}{\delta \phi(x)} \frac{\delta^{2} S\left[\phi_{\theta}\right]}{\delta \phi_{\theta}(z) \delta \phi_{\theta}(w)} \frac{\delta \phi_{\theta}(w)}{\delta \phi(y)}\right|_{\phi_{*}} .
$$

As a consequence, provided the duality transformation induced by the background $\phi_{*}$ is invertible, the linearized equation of motion (6.71) for the perturbation $\chi(x)$ around the background of $\phi_{*}$ in the dual theory with action $S_{\theta}$ is equivalent to

$$
\left.\int \mathrm{d}^{d} y \frac{\delta^{2} S\left[\phi_{\theta}\right]}{\delta \phi_{\theta}(x) \delta \phi_{\theta}(y)}\right|_{\left(\phi_{*}\right)_{\theta}} \chi_{\theta}(y)=0 .
$$

Here we have defined (cf. (6.9))

$$
\chi_{\theta}(x)=\left.\int \mathrm{d}^{d} z \frac{\delta \phi_{\theta}(x)}{\delta \phi(z)}\right|_{\phi_{*}} \chi(z)=\int \mathrm{d}^{d} z \delta^{(d)}\left(X\left[\phi_{*}\right](x)-z\right) \chi(z)=\chi\left(X\left[\phi_{*}\right](x)\right) .
$$

$\chi_{\theta}(x)$ is therefore a solution of the linearized fluctuation equation in the original theory around the classical configuration $\left(\phi_{*}\right)_{\theta}(x)$. Formula (6.78) is thus the desired duality transformation for the perturbations $\chi(x)$.

Up to now we have interpreted all the duality transformations actively, i.e. we assumed that both the original and dual theories live on the same Minkowski spacetime and

\footnotetext{
${ }^{26}$ Strictly speaking, the influence cone is given by the equation $g_{\mu \nu}^{-1}\left[\phi_{*}\right] N^{\mu} N^{\nu}=0$ where $g_{\mu \nu}^{-1}\left[\phi_{*}\right]$ is inverse to $g^{\mu \nu}\left[\phi_{*}\right]$. However according to (6.69) the metric $G_{\mu \nu}^{-1}\left[\phi_{*}\right]$ is conformally equivalent to $g_{\mu \nu}^{-1}\left[\phi_{*}\right]$.

${ }^{27}$ Here we tacitly assume the invertibility of the dual transformation.
} 
the fields $\phi_{*}(x), \chi(x)$ and $\left(\phi_{*}\right)_{\theta}(x), \chi_{\theta}(x)$ represent different field configurations within two different theories expressed in terms of the same Minkowski coordinates $x$. However, due to the geometrical nature of the fluctuation action and the corresponding equation of motion (6.68), (6.72) we can also change the point of view and interpret the duality transformation of $\chi(x)$ passively. Note, that we can rewrite it equivalently as a transformation of both field and coordinates

$$
x_{\theta}=x-2 \theta \partial \phi_{*}(x), \quad \chi_{\theta}\left(x_{\theta}\right)=\chi(x) .
$$

Unlike the original duality (6.4), the coordinate transformation here does not depend on the transformed function $\chi(x)$ and is fixed by the classical solution $\phi_{*}(x)$. Therefore, in the the linearized theory of perturbation the duality can be interpreted as a special coordinate transformation (6.70) under which the effective (inverse) metric $G_{\theta}^{\mu \nu}\left[\phi_{*}\right]$ corresponding to the dual action ${ }^{28} S_{\theta}$ transforms according to

$$
G_{\theta}^{\mu \nu}\left[\phi_{*}\right]^{\prime}\left(x_{\theta}\right)=G_{\theta}^{\rho \sigma}\left[\phi_{*}\right](x) \partial_{\rho} x_{\theta}^{\mu} \partial_{\sigma} x_{\theta}^{\nu}=G^{\rho \sigma}\left[\left(\phi_{*}\right)_{\theta}\right]\left(x_{\theta}\right)
$$

where $G^{\rho \sigma}\left[\left(\phi_{*}\right)_{\theta}\right]$ refers to the original action $S$. Under this interpretation, the fields $\chi(x)$ and $\chi_{\theta}\left(x_{\theta}\right)$ represent the same physical (geometrical) object within the same theory expressed in terms of two different systems of coordinates on a (generally curved) effective spacetime with the (inverse) metric $G_{\theta}^{\mu \nu}\left[\phi_{*}\right]$. Especially, the influence cone (6.74) does not change within the passive interpretation.

On the other hand, within the active interpretation, when both $x$ and $x_{\theta}$ are Minkowski coordinates and the original and dual theories are taken to be different, we can relate the influence cones in both theories. According to (6.80) we get

$$
G_{\mu \nu}^{-1}\left[\phi_{*}\right]_{\theta}(x) N^{\mu} N^{\nu}=G_{\rho \sigma}^{-1}\left[\left(\phi_{*}\right)_{\theta}\right]\left(x_{\theta}\right) \partial_{\mu} x_{\theta}^{\rho} \partial_{\nu} x_{\theta}^{\sigma} N^{\mu} N^{\nu} .
$$

Therefore, provided $N$ is a propagation vector forming the influence cone at point $x$ in the dual theory, we get propagation vector $N_{\theta}$ at point $x_{\theta}$ in the original one as

$$
N_{\theta}^{\mu}=N^{\sigma} \partial_{\sigma} x_{\theta}^{\mu}(x)=N^{\sigma}-2 \theta N^{\sigma} \partial_{\sigma} \partial^{\mu} \phi_{*}(x) .
$$

The actively interpreted duality transformation can thus deform the influence cone and e.g. connect healthy (sub)luminally propagating theory with pathological superluminally propagating one.

This might seem to be a paradox, however, in fact there is nothing unnatural in it. Let us remind the discussion of the duality of observables in the subsection 6.2. Provided we would like to describe the small fluctuations in the original theory in terms of the dual theory, we have to use the corresponding dual observable to the fluctuation operator $\delta^{2} S[\phi] / \delta \phi \delta \phi$ in the sense of the definition (6.62). The point is that, while the actions $S[\phi]$ and $S_{\theta}[\phi]$ are dual observables in the sense of (6.62), the corresponding fluctuation operators $\delta^{2} S[\phi] / \delta \phi \delta \phi$ and $\delta^{2} S_{\theta}[\phi] / \delta \phi \delta \phi$ are not. Note that the dual observable $\left(\delta^{2} S[\phi] / \delta \phi \delta \phi\right)_{\theta}$ to $\delta^{2} S[\phi] / \delta \phi \delta \phi$ is according to $(6.62)$

$$
\left(\frac{\delta^{2} S}{\delta \phi(x) \delta \phi(y)}\right)_{\theta}[\phi]=\left(\frac{\delta^{2} S}{\delta \phi(x) \delta \phi(y)}\right)\left[\phi_{\theta}[\phi]\right]=-g\left[\phi_{\theta}[\phi]\right]^{\mu \nu}(x) \partial_{\mu} \partial_{\nu} \delta^{(d)}(x-y)
$$

\footnotetext{
${ }^{28}$ I.e. it is given by (6.69) and (6.66) with the substitution $d_{n} \rightarrow d_{n}(\theta)$.
} 
and for the background $\phi_{*} \in \Sigma_{\theta}$ within the dual theory generates the same effective metric $G_{\rho \sigma}^{-1}\left[\left(\phi_{*}\right)_{\theta}\right](x)$ as $\left(\phi_{*}\right)_{\theta} \in \Sigma$ in the original theory. On the other hand the fluctuation operator in the dual theory has the form

$$
\frac{\delta^{2} S_{\theta}[\phi]}{\delta \phi(x) \delta \phi(y)} \equiv-g_{\theta}^{\alpha \beta}[\phi](x) \partial_{\mu} \partial_{\nu} \delta^{(d)}(x-y)
$$

where $g_{\theta}^{\alpha \beta}[\phi]$ is given by formula (6.66) with $d_{n} \rightarrow d_{n}(\theta)$. The corresponding effective metric is $(\text { cf. }(6.80))^{29}$

$$
\begin{aligned}
G_{\mu \nu}^{-1}\left[\phi_{*}\right]_{\theta}(x)= & G_{\rho \sigma}^{-1}\left[\left(\phi_{*}\right)_{\theta}\right]\left(x_{\theta}(x)\right) \partial_{\mu} x_{\theta}^{\rho}(x) \partial_{\nu} x_{\theta}^{\sigma}(x) \\
= & \left(\eta_{\mu}^{\rho}-2 \theta \partial_{\mu} \partial^{\rho} \phi_{*}(x)\right)\left(\eta_{\nu}^{\sigma}-2 \theta \partial_{\nu} \partial^{\sigma} \phi_{*}(x)\right) \\
& \times \sum_{n=0}^{\infty} \frac{(-2 \theta)^{n}}{n !} \partial^{\mu_{1}} \phi_{*}(x) \ldots \partial^{\mu_{n}} \phi_{*}(x) \partial_{\mu_{1}} \ldots \partial_{\mu_{n}} G_{\rho \sigma}^{-1}\left[\left(\phi_{*}\right)_{\theta}\right](x),
\end{aligned}
$$

which differs from $G_{\rho \sigma}^{-1}\left[\left(\phi_{*}\right)_{\theta}\right](x)$ at the same point. As a result, the right dual observable to $\delta^{2} S[\phi] / \delta \phi \delta \phi$ differs form $\delta^{2} S_{\theta}[\phi] / \delta \phi \delta \phi$. The fluctuation operators $\delta^{2} S[\phi] / \delta \phi \delta \phi$ and $\delta^{2} S_{\theta}[\phi] / \delta \phi \delta \phi$ represent therefore two different observables in two different theories. Because in the linearized case the small fluctuations correspond to the zero modes of these fluctuation operators, we cannot in general expect that they must necessarily propagate with the same (front, group) velocity.

On the other hand, we can construct a solution of the linearized fluctuation equation in the theory with action $S[\phi]$ around the classical solution $\left(\phi_{*}\right)_{\theta}$ from the corresponding solution of the dual theory with action $S_{\theta}[\phi]$ by means of duality transformation (6.78), (6.79). This means, that the covariant phase spaces of both linearized theories of fluctuations are dual to each other, and using the dual observables $\mathcal{O}_{\theta}[\chi]=\mathcal{O}\left[\chi_{\theta}[\chi]\right]$ in the framework of the dual theory enables us to get results for the observables $O[\chi]$ in the original theory. See also appendix $\mathrm{C}$ for further details.

In the following subsections we show two examples of the duality transformation of the perturbation of the classical solutions. We will demonstrate explicitly the usefulness of the formula (6.78) for solving the linearized fluctuation equation of motion.

\subsubsection{Fluctuations of the plane wave background}

The first example is the fluctuation of the plane wave background (see e.g. [43, 9]). In this case the background $\phi_{*}$ has the form

$$
\phi_{*}(x)=F(n \cdot x)
$$

\footnotetext{
${ }^{29}$ Using the inversion of the formula (6.76) (cf. also (6.9) and definition of $X[\phi](x)$ ) we can also relate directly the fluctuation operators for $\phi_{*} \in \Sigma_{\theta}$

$$
\left(\frac{\delta^{2} S}{\delta \phi(x) \delta \phi(y)}\right)_{\theta}\left[\phi_{*}\right]=-\operatorname{det}^{-1}\left(\frac{\partial x_{\theta}}{\partial x}\right) \partial_{\alpha} x_{\theta}^{\mu} \partial_{\beta} x_{\theta}^{\nu} g_{\theta}^{\alpha \beta}\left[\phi_{*}\right]\left(X\left[\phi_{*}\right](x)\right) \partial_{\mu} \partial_{\nu} \delta^{(d)}(x-y) .
$$

Note that, while $\delta^{2} S_{\theta}[\phi] / \delta \phi \delta \phi$ is a local functional of $\phi_{*}$ depending only on the second derivatives of $\phi_{*}$ at point $x$, the dual $\left(\delta^{2} S[\phi] / \delta \phi \delta \phi\right)_{\theta}$ is nonlocal and can be formally expanded into an infinite series which includes all the derivatives of $\phi_{*}$ at $x$. It is however a local functional of $\phi_{\theta}\left[\phi_{*}\right]$.
} 
where $F(\cdot)$ is some twice differentiable function. For light-like $n=(1, \mathbf{n})$ with $\mathbf{n}^{2}=1$, such $\phi_{*}(x)$ is a classical solution of the general Galileon equation of motion ${ }^{30}(2.7)$. The reason is that, for the ansatz (6.87), the problem is effectively one-dimensional and therefore the interaction terms in the equation of motion vanish automatically. ${ }^{31}$

The linearized equation of motion for the fluctuation of this background has the form (6.71), explicitly

$$
\left[d_{2} \square-d_{3} F^{\prime \prime}(n \cdot x)(n \cdot \partial)^{2}\right] \chi(x)=0
$$

and only the cubic coupling matters. The effective metric is in this case

$$
G_{\mu \nu}^{-1}\left[\phi_{*}\right]=\frac{1}{d_{2}}\left(\eta_{\mu \nu}+\frac{d_{3}}{d_{2}} F^{\prime \prime}(n \cdot x) n_{\mu} n_{\nu}\right), \quad G\left[\phi_{*}\right]=-\frac{1}{d_{2}^{4}}
$$

The linearized theory of fluctuations on the plane wave background is known to be a prominent example of a possible classical superluminal propagation, which is sometimes interpreted as a pathology of the Galileon theory. In the short wave-length (eikonal) approximation $\chi=A \exp (\mathrm{i} S)$ (where $S$ is assumed to be large) we get

$$
d_{2} \partial S \cdot \partial S-d_{3} F^{\prime \prime}(n \cdot x)(n \cdot \partial S)^{2}=0 .
$$

Denoting $\partial S=(\omega(\mathbf{k}), \mathbf{k})$ we get for the wave front velocity

$$
v_{\text {front }}^{2}=\frac{\omega(\mathbf{k})^{2}}{\mathbf{k}^{2}}=1+\frac{d_{3}}{d_{2}} F^{\prime \prime}(n \cdot x)(n \cdot \partial S)^{2},
$$

and thus the equation (6.88) leads locally to superluminal propagation of the fluctuations provided (see also [9] for more details)

$$
\frac{d_{3}}{d_{2}} F^{\prime \prime}(n \cdot x)>0
$$

In this case the lightcone corresponding to the effective metric (6.89) is wider then the Minkowski one.

Here we will show that duality can help us to find easily the explicit solution of (6.88). The duality transformation induced by $\phi_{*}(x)=F(n \cdot x)$ reads

$$
\begin{aligned}
x_{\theta} & =x-2 \theta n F^{\prime}(n \cdot x) \\
\left(\phi_{*}\right)_{\theta}\left(x_{\theta}\right) & =F(n \cdot x) .
\end{aligned}
$$

\footnotetext{
${ }^{30}$ Here we take $d_{1}=0$ but the other $d_{i}$ we assume to be arbitrary.

${ }^{31}$ Let us remind that the equation of motion reads

$$
\sum_{n=1}^{d+1} n d_{n} \mathcal{L}_{n-1}^{\mathrm{der}}\left(\partial \partial \phi_{*}\right)=0
$$

We have for $n>2$

$$
\mathcal{L}_{n-1}^{\text {der }}\left(\partial \partial \phi_{*}\right)=\mathcal{L}_{n-1}^{\text {der }}\left(n n F^{\prime \prime}(n \cdot x)\right)=0 .
$$

For $n=2$

$$
\mathcal{L}_{1}^{\text {der }}\left(n n F^{\prime \prime}(n \cdot x)\right)=(-1)^{d-1}(d-1) !(n \cdot n) F^{\prime \prime}(n \cdot x)
$$
}

which requires $n$ to be light-like $n^{2}=0$. 
Therefore

$$
n \cdot x=n \cdot x_{\theta}
$$

and $\left(\phi_{*}\right)_{\theta}(x)=F(n \cdot x)$, while the fluctuations $\chi(x)$ in the dual theory are governed by the equation (6.88) with the exchange $d_{2,3} \rightarrow d_{2,3}(\theta)$. With the choice

$$
\theta_{*}=\frac{d_{3}}{4 d_{2}}
$$

for the duality transformation (5.25) we get $d_{3}\left(\theta_{*}\right)=0$ and the fluctuation equation is simply $\square \chi(x)=0$ with general solution

$$
\chi(x)=\int \frac{\mathrm{d}^{3} \mathbf{k}}{(2 \pi)^{3} 2|\mathbf{k}|}[a(\mathbf{k}) \exp (-\mathrm{i} k \cdot x)+h . c]
$$

where $k=(|\mathbf{k}|, \mathbf{k})$. The dual $\chi_{\theta_{*}}(x)$ to $\chi(x)$ given by (6.78) yields then the solution of the general fluctuation equation (6.88). With help of (6.95) we can easily find the inversion $X\left[\phi_{*}\right](x)$ of $(6.93)$, namely

$$
X\left[\phi_{*}\right](x)=x+2 \theta n F^{\prime}(n \cdot x)
$$

and using the general prescription (6.78) we get finally the general solution of (6.88) in the form

$$
\chi_{\theta_{*}}(x)=\int \frac{\mathrm{d}^{3} \mathbf{k}}{(2 \pi)^{3} 2|\mathbf{k}|}\left\{a(\mathbf{k}) \chi_{\mathbf{k}}(x)+\text { h.c. }\right\},
$$

where the basis of the solutions is

$$
\chi_{\mathbf{k}}(x)=\exp \left[-\mathrm{i} k \cdot X\left[\phi_{*}\right](x)\right]=\exp \left[-\mathrm{i} k \cdot x-2 \mathrm{i} \theta_{*}(n \cdot k) F^{\prime}(n \cdot x)\right] .
$$

Let us now discuss the physical properties of this solution, namely the conditions under which we get superluminal propagation. Assume that the coefficient function $a(\mathbf{k})$ has a sharp peak at $\mathbf{k}=\overline{\mathbf{k}}$ and is nonzero only in as small vicinity of this point. Then we can write approximately

$$
\chi_{\theta_{*}}(x) \approx \mathrm{e}^{-\mathrm{i} \bar{k} \cdot X\left[\phi_{*}\right]} \mathcal{A}\left(\mathbf{X}\left[\phi_{*}\right]-\widehat{\mathbf{k}} X^{0}\left[\phi_{*}\right]\right)+\text { h.c. }
$$

where $\bar{k}=(|\overline{\mathbf{k}}|, \overline{\mathbf{k}}), \widehat{\mathbf{k}}=\overline{\mathbf{k}} /|\overline{\mathbf{k}}|$ and the shape of the wave packet is given by

$$
\mathcal{A}(\mathbf{y})=\int \frac{\mathrm{d}^{3} \mathbf{k}}{(2 \pi)^{3} 2|\mathbf{k}|} a(\mathbf{k}+\overline{\mathbf{k}}) \mathrm{e}^{\mathrm{i} \mathbf{k} \cdot \mathbf{y}} .
$$

The group velocity can be now obtained in a standard way by differentiating the equation

$$
\mathbf{X}\left[\phi_{*}\right]-\widehat{\mathbf{k}} X^{0}\left[\phi_{*}\right]=\text { const. }
$$

with respect to $t \equiv x^{0}$. After some algebra (see appendix D) we find

$$
\mathbf{v}_{\text {group }}(x)=\frac{\widehat{\mathbf{k}}-2 \theta_{*}(1-\mathbf{n} \cdot \widehat{\mathbf{k}}) F^{\prime \prime}(n \cdot x) \mathbf{n}}{1-2 \theta_{*} F^{\prime \prime}(n \cdot x)(1-\mathbf{n} \cdot \widehat{\mathbf{k}})}
$$


and thus

$$
v_{\text {group }}(x)=\left(1+\frac{4 \theta_{*} F^{\prime \prime}(n \cdot x)(1-\mathbf{n} \cdot \widehat{\mathbf{k}})^{2}}{\left[1-2 \theta_{*} F^{\prime \prime}(n \cdot x)(1-\mathbf{n} \cdot \widehat{\mathbf{k}})\right]^{2}}\right)^{1 / 2} .
$$

Therefore the superluminal propagation is possible in the space-time regions with $\theta_{*} F^{\prime \prime}(n$. $x)=3 d_{3} F^{\prime \prime}(n \cdot x)>0$. The phase velocity is given by

$$
v_{\text {phase }}(x)=\frac{\frac{\mathrm{d}}{\mathrm{d} t} \bar{k} \cdot X\left[\phi_{*}\right]}{\left|\nabla \bar{k} \cdot X\left[\phi_{*}\right]\right|}=\left(1-\frac{4 \theta_{*} F^{\prime \prime}(n \cdot x)(1-\mathbf{n} \cdot \widehat{\mathbf{k}})^{2}}{\left[1+2 \theta_{*} F^{\prime \prime}(n \cdot x)(1-\mathbf{n} \cdot \widehat{\mathbf{k}})\right]^{2}}\right)^{-1 / 2}
$$

and is superluminal under the same condition as $v_{\text {group }}$.

The explicit knowledge of the basis $\chi_{\mathbf{k}}(x)$ allows us to discuss also the quantum aspects of the fluctuations on the operator level. Because of a special role of the variable $n \cdot x$, which naturally plays a role of the evolution parameter in the problem, the most convenient prescription for the quantization of the field $\chi$ is the Dirac front-form one. Let us introduce the light cone coordinates as

$$
x^{+}=n \cdot x, \quad x^{-}=\widetilde{n} \cdot x, \quad \mathbf{x}_{\perp}=\mathbf{x}-\mathbf{n}(\mathbf{n} \cdot \mathbf{x})
$$

where $\widetilde{n}=(1,-\mathbf{n})$ and analogously for any other vector. Then e.g. the solutions $\chi_{\mathbf{k}}(x)$ can be rewritten in the form

$$
\chi_{\mathbf{k}}(x)=\exp \left[-\frac{\mathrm{i}}{2} k^{-} x^{+}-\frac{\mathrm{i}}{2} k^{+} x^{-}+\mathrm{i} \mathbf{k}_{\perp} \cdot \mathbf{x}_{\perp}-2 \mathrm{i} \theta_{*} k^{+} F^{\prime}\left(x^{+}\right)\right]
$$

and the condition on the vector $k$ to be on-shell and positive-energy is then expressed as

$$
k^{-}=\frac{\mathbf{k}_{\perp}^{2}}{k^{+}}, \quad k^{+} \geq 0 .
$$

It is easy to prove that the elements of the basis $\chi_{\mathbf{k}}(x)$ are orthogonal with respect to the indefinite scalar product defined on the solution of (6.88) $\mathrm{as}^{32}$

$$
\left\langle\chi_{1}, \chi_{2}\right\rangle=\mathrm{i} \int_{x^{+}=\text {const. }} \mathrm{d} x^{-} \mathrm{d}^{2} \mathbf{x}_{\perp} \chi_{1}^{*} \overleftrightarrow{\partial_{-}} \chi_{2}
$$

namely

$$
\begin{aligned}
& \left\langle\chi_{\mathbf{k}}, \chi_{\mathbf{q}}\right\rangle=-\left\langle\chi_{\mathbf{k}}^{*}, \chi_{\mathbf{q}}^{*}\right\rangle=(2 \pi)^{3} 2 k^{+} \delta\left(k^{+}-q^{+}\right) \delta^{(2)}\left(\mathbf{k}_{\perp}-\mathbf{q}_{\perp}\right) \\
& \left\langle\chi_{\mathbf{k}}^{*}, \chi_{\mathbf{q}}\right\rangle=\left\langle\chi_{\mathbf{k}}, \chi_{\mathbf{q}}^{*}\right\rangle=0 .
\end{aligned}
$$

Let us write the operator solution $\widehat{\chi}(x)$ of the equation (6.88) in the form (see (6.99) and (6.100))

$$
\widehat{\chi}(x)=\int_{k^{+}>0} \frac{\mathrm{d} k^{+} \mathrm{d}^{2} \mathbf{k}_{\perp}}{(2 \pi)^{3} 2 k^{+}}\left(a\left(k^{+}, \mathbf{k}_{\perp}\right) \chi_{\mathbf{k}}(x)+a^{+}\left(k^{+}, \mathbf{k}_{\perp}\right) \chi_{\mathbf{k}}^{*}(x)\right) .
$$

\footnotetext{
${ }^{32}$ For the solutions $\chi_{1,2}$ of the fluctuation equation of motion the scalar product is independent on the choice of $x^{+}$.
} 
According to the general quantization prescription, the operators $\widehat{\chi}(x)$ and their canonically conjugated momenta in the front-form formalism

$$
\widehat{\pi}(x)=2 \partial_{-} \widehat{\chi}(x)
$$

have to satisfy the canonical commutation relations

$$
\begin{aligned}
& {[\widehat{\chi}(x), \widehat{\chi}(y)]_{x^{+}=y^{+}}=-\frac{\mathrm{i}}{4} \varepsilon\left(x^{-}-y^{-}\right) \delta^{(2)}\left(\mathbf{x}_{\perp}-\mathbf{y}_{\perp}\right)} \\
& {[\widehat{\chi}(x), \widehat{\pi}(y)]_{x^{+}=y^{+}}=\mathrm{i} \delta\left(x^{-}-y^{-}\right) \delta^{(2)}\left(\mathbf{x}_{\perp}-\mathbf{y}_{\perp}\right) .}
\end{aligned}
$$

This implies with help of (6.110) the canonical commutation relations

$$
\begin{aligned}
{\left[a\left(k^{+}, \mathbf{k}_{\perp}\right), a^{+}\left(q^{+}, \mathbf{q}_{\perp}\right)\right] } & =(2 \pi)^{3} 2 k^{+} \delta\left(k^{+}-q^{+}\right) \delta^{(2)}\left(\mathbf{k}_{\perp}-\mathbf{q}_{\perp}\right) \\
{\left[a\left(k^{+}, \mathbf{k}_{\perp}\right), a\left(q^{+}, \mathbf{q}_{\perp}\right)\right] } & =\left[a^{+}\left(k^{+}, \mathbf{k}_{\perp}\right), a^{+}\left(q^{+}, \mathbf{q}_{\perp}\right)\right]=0 .
\end{aligned}
$$

Now it is easy to calculate the commutator of the fields with the result

$$
[\widehat{\chi}(x), \widehat{\chi}(y)]=-\frac{i}{(2 \pi)} \varepsilon\left(x^{+}-y^{+}\right) \delta(\lambda)
$$

where $\varepsilon(z)=\theta(z)-\theta(-z)$ is the sign function and

$$
\lambda=(x-y)^{2}+4 \theta_{*}\left(x^{+}-y^{+}\right)\left(F^{\prime}\left(x^{+}\right)-F^{\prime}\left(y^{+}\right)\right) .
$$

The commutator is nonzero only for

$$
(x-y)^{2}=-4 \theta_{*}\left(x^{+}-y^{+}\right)^{2} \frac{F^{\prime}\left(x^{+}\right)-F^{\prime}\left(y^{+}\right)}{x^{+}-y^{+}}=-4 \theta_{*}\left(x^{+}-y^{+}\right)^{2} F^{\prime \prime}\left(\xi^{+}\right)
$$

where $\xi^{+}$is located between $x^{+}$and $y^{+}$. Provided $\theta_{*} F^{\prime \prime}\left(\xi^{+}\right)>0$, the commutator is nonzero also outside the light cone and causality is violated.

The commutation relations (6.114) can be represented on the Fock space built on the vacuum state $|0\rangle$ which is annihilated by the operators $a\left(k^{+}, \mathbf{k}_{\perp}\right)$. Let us suppose that there exist finite limits

$$
\lim _{x^{+} \rightarrow \pm \infty} F^{\prime}\left(x^{+}\right) \equiv \psi_{ \pm}
$$

Then in the limit $t \rightarrow \pm \infty$, ( $\mathbf{x}$ fixed) we get

$$
\widehat{\chi}(x) \rightarrow \widehat{\chi}_{\text {out,in }}(x)=\int_{k^{+}>0} \frac{\mathrm{d} k^{+} \mathrm{d}^{2} \mathbf{k}_{\perp}}{(2 \pi)^{3} 2 k^{+}}\left(a_{\text {out,in }}\left(k^{+}, \mathbf{k}_{\perp}\right) \mathrm{e}^{-\mathrm{i} k \cdot x}+a_{\text {out,in }}^{+}\left(k^{+}, \mathbf{k}_{\perp}\right) \mathrm{e}^{\mathrm{i} k \cdot x}\right) .
$$

Here we have identified the out and in creation and annihilation operators

$$
\begin{aligned}
& a_{\text {out }, \text { in }}\left(k^{+}, \mathbf{k}_{\perp}\right)=a\left(k^{+}, \mathbf{k}_{\perp}\right) \exp \left[-2 \mathrm{i} \theta_{*} k^{+} \psi_{ \pm}\right] \\
& a_{\text {out }, \text { in }}^{+}\left(k^{+}, \mathbf{k}_{\perp}\right)=a^{+}\left(k^{+}, \mathbf{k}_{\perp}\right) \exp \left[2 \mathrm{i} \theta_{*} k^{+} \psi_{ \pm}\right] .
\end{aligned}
$$

Note that these operators satisfy again the commutation relations (6.114). The fields $\widehat{\chi}_{\text {out,in }}(x)$ are therefore free fields which create asymptotic in and out states from the Fock vacuum

$$
\left.\mid \mathbf{k}_{(1)}, \ldots, \mathbf{k}_{(m)} ; \text { out, in }\right\rangle=a_{\text {out }, \text { in }}^{+}\left(k_{(1)}^{+}, \mathbf{k}_{(1) \perp}\right) \ldots a_{\text {out }, \text { in }}^{+}\left(k_{(m)}^{+}, \mathbf{k}_{(m) \perp}\right)|0\rangle
$$


These are eigenstates of the momentum operators

$$
\begin{aligned}
P^{+} & =\int_{k^{+}>0} \frac{\mathrm{d} k^{+} \mathrm{d}^{2} \mathbf{k}_{\perp}}{(2 \pi)^{3} 2 k^{+}} k^{+} a^{+}\left(k^{+}, \mathbf{k}_{\perp}\right) a\left(k^{+}, \mathbf{k}_{\perp}\right) \\
P^{-} & =\int_{k^{+}>0} \frac{\mathrm{d} k^{+} \mathrm{d}^{2} \mathbf{k}_{\perp}}{(2 \pi)^{3} 2 k^{+}} \frac{\mathbf{k}_{\perp}^{2}}{k^{+}} a^{+}\left(k^{+}, \mathbf{k}_{\perp}\right) a\left(k^{+}, \mathbf{k}_{\perp}\right) \\
\mathbf{P}_{\perp} & =\int_{k^{+}>0} \frac{\mathrm{d} k^{+} \mathrm{d}^{2} \mathbf{k}_{\perp}}{(2 \pi)^{3} 2 k^{+}} \mathbf{k}_{\perp} a^{+}\left(k^{+}, \mathbf{k}_{\perp}\right) a\left(k^{+}, \mathbf{k}_{\perp}\right)
\end{aligned}
$$

with eigenvalues $\sum_{i=1}^{m} k_{(i)}$ where $k_{(i)}=\left(k_{(i)}^{+}, k_{(i)}^{-}, \mathbf{k}_{(i) \perp}\right)$ satisfying $k_{(i)}^{2}=0$ and correspond therefore to the $m$ non-interacting massless excitations. The $S$-matrix defined as

$$
a_{\text {out }}\left(k^{+}, \mathbf{k}_{\perp}\right)=a_{\text {in }}\left(k^{+}, \mathbf{k}_{\perp}\right) \exp \left[2 \mathrm{i} \theta_{*} k^{+}\left(\psi_{-}-\psi_{+}\right)\right] \equiv S^{+} a_{\text {in }}\left(k^{+}, \mathbf{k}_{\perp}\right) S
$$

is then expressed simply as a translation in the $x^{-}$direction

$$
S=\exp \left[2 \mathrm{i} \theta_{*} P^{+}\left(\psi_{-}-\psi_{+}\right)\right] .
$$

The only nontrivial connected scattering amplitude is the two-point one ${ }^{33}$

$$
\mathcal{M}\left(\mathbf{k}, \mathbf{k}^{\prime}\right)=(4 \pi)^{2} \frac{\mathrm{e}^{2 \mathrm{i} \delta_{\mathbf{k}}}-1}{2 \mathrm{i}|\mathbf{k}|} \delta^{(2)}\left(\widehat{\mathbf{k}}-\widehat{\mathbf{k}}^{\prime}\right)
$$

which describes scattering of the individual excitations on the background resulting in a phase shift $\delta_{\mathbf{k}}=\theta_{*} k^{+}\left(\psi_{-}-\psi_{+}\right)$. This amplitude can be also obtained by means of summation of the perturbative series generated by the Feynman rules depicted in figure 3 (see appendix E).

Note that the $S$ matrix is trivial for $\left(\psi_{-}-\psi_{+}\right)=0$, i.e. especially when $F\left(x^{+}\right)$has compact support. This is in accord with the discussion in [40], where it has been demonstrated that for localized classical background the displacement of the null geodetics with respect to the effective metric vanish asymptotically.

To conclude, we have shown that within the original theory with $d_{3} \neq 0$ the fluctuations in general interact with the plane wave background and might be scattered by it provided its profile $F\left(x^{+}\right)$does not fall rapidly enough for $x^{+} \rightarrow \infty$. Also the commutator of the operators $\widehat{\chi}(x)$ might locally violate causality when $\theta_{*} F^{\prime \prime}\left(x^{+}\right)>0$ in some region. This might be felt as a paradox since for the dual theory for which $d_{3}=0$ the linearized fluctuation theory is free, causal, and its $S$ matrix is trivial. However, we are comparing here two different theories the relation of which must be taken with care. As we have argued, only comparison of dual observables makes sense. E.g. provided we would like to describe the correlators of the fluctuations of the original theory using the dual (free) one, we have not to use the free operators

$$
\widehat{\chi}_{0}(x)=\int_{k^{+}>0} \frac{\mathrm{d} k^{+} \mathrm{d}^{2} \mathbf{k}_{\perp}}{(2 \pi)^{3} 2 k^{+}}\left(a\left(k^{+}, \mathbf{k}_{\perp}\right) \mathrm{e}^{-\mathrm{i} k \cdot x}+a^{+}\left(k^{+}, \mathbf{k}_{\perp}\right) \mathrm{e}^{\mathrm{i} k \cdot x}\right),
$$

\footnotetext{
${ }^{33}$ Here we use the normalization

$$
S_{f i}=\left\langle\mathbf{k}^{\prime} \mid \mathbf{k}\right\rangle+2 \pi \mathrm{i} \delta\left(E^{\prime}-E\right) \mathcal{M}\left(\mathbf{k}^{\prime}, \mathbf{k}\right)
$$
}



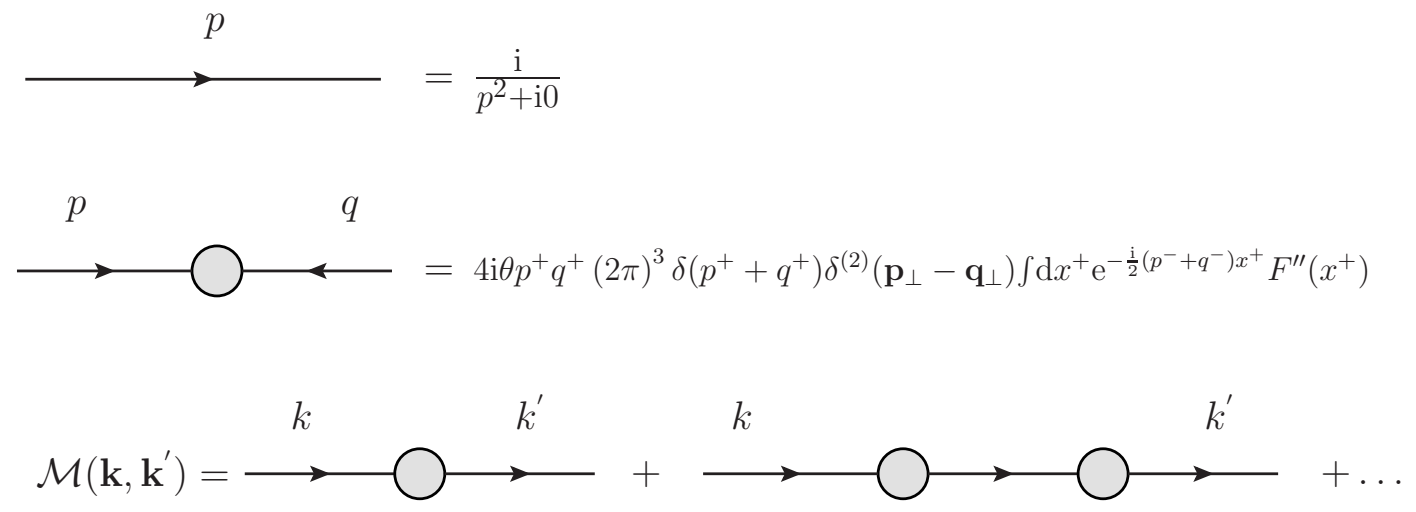

Figure 3. The Feynman rules for the perturbative calculation of the one-particle amplitude $\mathcal{M}\left(\mathbf{k}, \mathbf{k}^{\prime}\right)$ are given in the first two rows of this figure. This amplitude is given by the sum of Feynman graphs depicted in the last row.

but their duals $\widehat{\chi}(x)$ given by (6.111), see also appendix C. These, though live on the same Hilbert space, are not unitarily equivalent ${ }^{34}$ to $\widehat{\chi}_{0}(x)$. Instead we have

$$
\widehat{\chi}(x)=\exp \left[-2 \mathrm{i} \theta_{*} P^{+} F^{\prime}\left(x^{+}\right)\right] \widehat{\chi}_{0}(x) \exp \left[2 \mathrm{i} \theta_{*} P^{+} F^{\prime}\left(x^{+}\right)\right]
$$

and thus the dual asymptotic in and out states defined by the dual operators $\widehat{\chi}(x)$ differ from that defined by $\widehat{\chi}_{0}(x)$ when $\left(\psi_{-}-\psi_{+}\right) \neq 0$.

\subsubsection{Fluctuations of the cylindrically symmetric solution}

As the second example let us consider briefly the fluctuations of the cylindrically symmetric static solution $\phi(z, \bar{z})$ discussed in the previous subsection. The matrix $g[\phi]^{\mu \nu}$ is in this case given in the form (see appendix $\mathrm{F}$ for details)

$$
\begin{aligned}
g[\phi]= & 12 d_{2}(\theta) \eta \\
& -12 d_{3}(\theta)\left(\begin{array}{cccc}
4 \partial \bar{\partial} \phi & 0 & 0 & 0 \\
0 & -4 \partial \bar{\partial} \phi & 0 & 0 \\
0 & 0 & (\partial-\bar{\partial})^{2} \phi & \mathrm{i}\left(\partial^{2}-\bar{\partial}^{2}\right) \phi \\
0 & 0 & \mathrm{i}\left(\partial^{2}-\bar{\partial}^{2}\right) \phi-(\partial+\bar{\partial})^{2} \phi
\end{array}\right) \\
& -24 d_{4}(\theta)\left(\begin{array}{cccc}
4\left[\partial^{2} \phi \bar{\partial}^{2} \phi-(\partial \bar{\partial} \phi)^{2}\right] & 0 & 0 & 0 \\
0 & -4\left[\partial^{2} \phi \bar{\partial}^{2} \phi-(\partial \bar{\partial} \phi)^{2}\right] & 0 & 0 \\
0 & 0 & 0 & 0 \\
0 & 0 & 0
\end{array}\right) .
\end{aligned}
$$

Due to the cylindrical symmetry $g[\phi]$ does not depend on the quintic coupling $d_{5}$. Let us now show the application of the duality to solving the general equation (6.71). As in the above examples, within the dual theory this equation simplifies. Setting $d_{2}=1 / 12$ as

\footnotetext{
${ }^{34}$ I.e. there does not exist any unitary operator $U$ on the Fock space such that $\widehat{\chi}(x)=U \widehat{\chi}_{0}(x) U^{+}$.
} 
usual and passing to the dual theory with $d_{3}(\theta)=0$ we get in terms of the invariant $I_{4}$ (cf. $(5.44))$

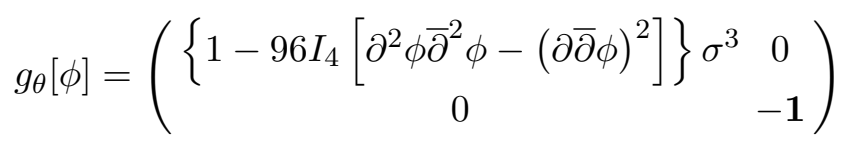

where $\sigma^{3}=\operatorname{diag}(1,-1)$ is the third Pauli matrix. Thus the equation $(6.71)$ becomes

$$
\left\{1-96 I_{4}\left[\partial^{2} \phi \bar{\partial}^{2} \phi-(\partial \bar{\partial} \phi)^{2}\right]\right\}\left(\ddot{\chi}-\chi^{\prime \prime}\right)-4 \partial \bar{\partial} \chi=0
$$

where $\chi \equiv \chi(t, x, z, \bar{z})$, dots and dashes means derivative with respect to $t$ and $x^{1}$ respectively. In the dual theory the static solution $\phi_{*}$ is given by (6.25) and for $z \neq 0$ we get explicitly the following dual fluctuation equation

$$
\left[1-6 I_{4}\left(\frac{\sigma}{\pi}\right)^{2} \frac{1}{(z \bar{z})^{2}}\right]\left(\ddot{\chi}-\chi^{\prime \prime}\right)-4 \partial \bar{\partial} \chi=0 .
$$

For

$$
1-6 I_{4}\left(\frac{\sigma}{\pi}\right)^{2} \frac{1}{(z \bar{z})^{2}}>0
$$

we can rewrite it equivalently as

$$
\chi_{; \mu}^{; \mu}=0
$$

where $\chi_{; \mu}^{; \mu}$ is the Laplace-Beltrami operator acting on $\chi$ and corresponding to the effective metric metric $G_{\mu \nu}^{-1}\left[\phi_{*}\right]_{\theta}$ given explicitly as

$$
\mathrm{d} s^{2}=\left(\mathrm{d} t^{2}-\mathrm{d} x^{2}\right)-\left[1-6 I_{4}\left(\frac{\sigma}{\pi}\right)^{2} \frac{1}{(z \bar{z})^{2}}\right] \mathrm{d} z \mathrm{~d} \bar{z} .
$$

Similarly to the case of the point-like source, the duality arguments help us to easily solve the equation (6.71) for the class of theories for which $I_{4}=0$. In such a case we have to solve a free equation $\square \chi=0$ with general solution (with luminal propagation)

$$
\chi(t, x, z, \bar{z})=\int \frac{\mathrm{d}^{3} \mathbf{k}}{(2 \pi)^{3} 2|\mathbf{k}|}\left\{a(\mathbf{k}) \exp \left[-\mathrm{i}\left(|\mathbf{k}| t-k^{1} x-z \bar{w}-\bar{z} w\right)\right]+h . c\right\}
$$

with $k=(|\mathbf{k}|, \mathbf{k})$ and

$$
\begin{aligned}
w & =\frac{1}{2}\left(k^{2}+\mathrm{i} k^{3}\right) \\
\bar{w} & =\frac{1}{2}\left(k^{2}-\mathrm{i} k^{3}\right) .
\end{aligned}
$$

Its dual $\chi_{\theta}$ given by (6.78) is then

$$
\chi_{\theta}\left(t, x^{1}, z, \bar{z}\right)=\int \frac{\mathrm{d}^{3} \mathbf{k}}{(2 \pi)^{3} 2|\mathbf{k}|}\left\{a(\mathbf{k}) \exp \left[-\mathrm{i}\left(|\mathbf{k}| t-k^{1} x-\zeta(z, \bar{z}) \bar{w}-\overline{\zeta(z, \bar{z})} w\right)\right]+h . c\right\}
$$


where (see (6.30) and (6.32))

$$
\begin{aligned}
& \zeta(z, \bar{z})=z\left(1+\frac{\sigma \theta}{\pi \rho(z, \bar{z})}\right)^{-1} \\
& \rho(z, \bar{z})=\frac{1}{2}\left(z \bar{z}-2 \frac{\sigma \theta}{\pi} \pm \sqrt{z \bar{z}\left(z \bar{z}-4 \frac{\sigma \theta}{\pi}\right)}\right) .
\end{aligned}
$$

According to the discussion in the introduction to this subsection, $\chi_{\theta}(t, x, z, \bar{z})$ is for $\theta=3 d_{3}$ the solution of the general fluctuation equation (6.71) with $g[\phi]$ given by (6.126) where $d_{4}=9 d_{3}^{2} / 2$.

\subsection{Hidden symmetries}

The Galileon duality often interrelates apparently very different theories. For instance, let us assume a Galileon theory with additional $Z_{2}$ symmetry which corresponds to the intrinsic parity, namely

$$
\phi \rightarrow \phi^{P}=-\phi .
$$

On the Lagrangian level this symmetry requires $d_{n}=0$ for all $n$ odd. Under the general duality transformation such a $Z_{2}$ invariant theory might be mapped onto a dual with some $d_{2 k-1} \neq 0$ and therefore the $Z_{2}$ symmetry ceases to be manifest in the dual theory. In this section we will study the way how the symmetries of the original Lagrangian are realized on the dual one.

Let us remind the definition of the dual action corresponding to the matrix $\alpha$

$$
S_{\alpha}[\phi]=S\left[\phi_{\alpha}\right]
$$

where $\phi_{\alpha}$ is the duality transformation of the field $\phi$ given by (cf. (4.19))

$$
\begin{aligned}
x_{\alpha}= & \alpha_{P P} x+\alpha_{P B} \partial \phi(x) \\
\phi_{\alpha}\left(x_{\alpha}\right)= & \operatorname{det}(\alpha) \phi(x) \\
& +\frac{1}{2}\left(\alpha_{P B} \alpha_{B B} \partial \phi(x) \cdot \partial \phi(x)+2 \alpha_{P B} \alpha_{B P} x \cdot \partial \phi(x)+\alpha_{P P} \alpha_{B P} x^{2}\right) \\
(\partial \phi)_{\alpha}\left(x_{\alpha}\right)= & \alpha_{B B} \partial \phi(x)+\alpha_{B P} x .
\end{aligned}
$$

Here we have denoted ${ }^{35}(\partial \phi)_{\alpha} \equiv \partial \phi_{\alpha} / \partial x_{\alpha}$. Within this notation the group property of the duality transformations can be formally expressed as

$$
\left(Y_{\alpha}\right)_{\beta}=Y_{\beta \cdot \alpha}, \quad Y=(x, \phi, \partial \phi) .
$$

The inverse of the transformation (6.138) has the same form with the exchange

$$
\alpha \rightarrow \alpha^{-1}=\operatorname{det}(\alpha)^{-1}\left(\begin{array}{cc}
\alpha_{B B} & -\alpha_{P B} \\
-\alpha_{B P} & \alpha_{P P}
\end{array}\right)
$$

\footnotetext{
${ }^{35}$ In the previous formulae and in what follows we suppress the Lorentz indices, the index $\alpha$ in $x_{\alpha}$ refers to the matrix $\alpha$.
} 
and can be written symbolically as

$$
Y=\left(Y_{\alpha}\right)_{\alpha^{-1}} \quad Y=(x, \phi, \partial \phi) .
$$

Using this notation the formula (6.137) can be rewritten in the form

$$
S[\phi]=S_{\alpha}\left[\phi_{\alpha^{-1}}\right]
$$

Now any transformation of the general form

$$
Y_{\alpha} \rightarrow\left(Y_{\alpha}\right)^{\prime}=\left(\mathcal{F}^{x}\left(Y_{\alpha}\right), \mathcal{F}^{\phi}\left(Y_{\alpha}\right), \mathcal{F}^{\partial \phi}\left(Y_{\alpha}\right)\right), \quad Y_{\alpha}=\left(x_{\alpha}, \phi_{\alpha}, \quad(\partial \phi)_{\alpha}\right),
$$

where $\mathcal{F}^{Y},(Y=x, \phi, \partial \phi)$, are local functions ${ }^{36}$ of $Y_{\alpha}$, is realized in terms of the variables $Y$ as

$$
Y \rightarrow Y^{\prime}=\left(\left(Y_{\alpha}\right)^{\prime}\right)_{\alpha^{-1}}=\left(\mathcal{F}^{x}\left(Y_{\alpha}\right), \mathcal{F}^{\phi}\left(Y_{\alpha}\right), \mathcal{F}^{\partial \phi}\left(Y_{\alpha}\right)\right)_{\alpha^{-1}}
$$

Provided the original action is symmetric with respect to the transformation (6.142) we have using (6.141)

$$
S_{\alpha}\left[\phi^{\prime}\right]=S_{\alpha}\left[\left(\left(\phi_{\alpha}\right)^{\prime}\right)_{\alpha^{-1}}\right]=S\left[\left(\phi_{\alpha}\right)^{\prime}\right]=S\left[\phi_{\alpha}\right]=S_{\alpha}[\phi]
$$

and the dual action is invariant with respect to (6.143).

Let us now give some explicit examples of these general formulae. The first example is the intrinsic parity transformation mentioned in the introduction to this section. In this case, the formula (6.143) simplifies considerably. Let us note that the intrinsic parity transformation (6.136) can be treated as a special case of the duality transformations (5.21) with a matrix

$$
\alpha_{P} \equiv \alpha_{S}(1,-1)=\left(\begin{array}{cc}
1 & 0 \\
0 & -1
\end{array}\right)
$$

Therefore $\phi^{P}=\phi_{\alpha_{P}}$ and (6.143) has the form

$$
Y^{P}=\left(\left(Y_{\alpha}\right)^{P}\right)_{\alpha^{-1}}=\left(Y_{\alpha_{P} \cdot \alpha}\right)_{\alpha^{-1}}=Y_{\alpha^{-1} \cdot \alpha_{P} \cdot \alpha}
$$

and the $Z_{2}$ symmetry is realized in the dual theory with action $S_{\alpha}[\phi]$ as a duality transformation associated with the matrix

$$
\beta_{P}(\alpha)=\alpha^{-1} \cdot \alpha_{P} \cdot \alpha=\operatorname{det}(\alpha)^{-1}\left(\begin{array}{cc}
\alpha_{P P} \alpha_{B B}+\alpha_{P B} \alpha_{B P} & 2 \alpha_{P B} \alpha_{B B} \\
-2 \alpha_{B P} \alpha_{P P} & -\alpha_{P P} \alpha_{B B}-\alpha_{P B} \alpha_{B P}
\end{array}\right),
$$

or explicitly

$$
\begin{aligned}
x^{P}= & \operatorname{det}(\alpha)^{-1}\left[\left(\alpha_{P P} \alpha_{B B}+\alpha_{P B} \alpha_{B P}\right) x+2 \alpha_{P B} \alpha_{B B} \partial \phi(x)\right] \\
\phi^{P}\left(x^{P}\right)= & -\phi(x)-\operatorname{det}(\alpha)^{-2}\left[\alpha_{P B} \alpha_{B B}\left(\alpha_{P P} \alpha_{B B}+\alpha_{P B} \alpha_{B P}\right) \partial \phi(x) \cdot \partial \phi(x)\right. \\
& \left.+4 \alpha_{P B} \alpha_{B P} \alpha_{P P} \alpha_{B B} x \cdot \partial \phi(x)+\alpha_{P P} \alpha_{B P}\left(\alpha_{P P} \alpha_{B B}+\alpha_{P B} \alpha_{B P}\right) x^{2}\right] \\
(\partial \phi)^{P}\left(x^{P}\right)= & -\operatorname{det}(\alpha)^{-1}\left[2 \alpha_{B P} \alpha_{P P} x+\left(\alpha_{P P} \alpha_{B B}+\alpha_{P B} \alpha_{B P}\right) \partial \phi(x)\right] .
\end{aligned}
$$

\footnotetext{
${ }^{36}$ Here $\mathcal{F}^{\partial \phi}$ is in fact not independent because it has to be compatible with the remaining two functions in such a way that $\mathcal{F}^{\partial \phi}(Y)=\partial^{\prime} \phi^{\prime}$.
} 
The transformation corresponding to the intrinsic parity is therefore realized in the dual theory non-linearly and non-locally as a simultaneous transformation of both space-time coordinates and fields.

In the same way we can find the dual realization of the Galileon symmetry (2.1). The general formula (6.143) reads in this case

$$
(x, \phi, \partial \phi)^{\prime}=\left(x_{\alpha}, \phi_{\alpha}+a+b \cdot x_{\alpha},(\partial \phi)_{\alpha}+b\right)_{\alpha^{-1}},
$$

or explicitly

$$
\begin{aligned}
x^{\prime} & =x-\operatorname{det}(\alpha)^{-1} \alpha_{P B} b \\
\phi^{\prime} & =\phi+a \operatorname{det}(\alpha)^{-1}-\frac{1}{2} \operatorname{det}(\alpha)^{-2} \alpha_{P B} \alpha_{P P} b^{2}+\operatorname{det}(\alpha)^{-1} \alpha_{P P} b \cdot x \\
(\partial \phi)^{\prime} & =\partial \phi+\operatorname{det}(\alpha)^{-1} \alpha_{P P} b .
\end{aligned}
$$

A dual Galileon transformation is therefore superposition of space-time translation and Galileon transformation with special values of parameters, as was recognized for the duality of the type (5.25) in [18].

Let us now discuss the space-time symmetries. The duality transformation respects the Lorentz symmetry, therefore its realization in the dual theory is the same as in the original one. Indeed, restoring the Lorentz structure in the matrix notation (5.1), (5.2) and (5.4) we can write

$$
\alpha=\left(\begin{array}{cc}
\alpha_{P P} & \alpha_{P B} \\
\alpha_{B P} & \alpha_{B B}
\end{array}\right) \otimes \mathbf{1}, \quad \widehat{\alpha}=\left(\begin{array}{cc}
\alpha_{B P} & 0 \\
0 & \alpha_{P B}
\end{array}\right) \otimes \mathbf{1}
$$

where the second factor $\mathbf{1} \equiv \delta_{\nu}^{\mu}$ in the tensor product acts to the vector indices of $X$. Using the same notation, the Lorentz transformation $\Lambda \equiv \Lambda_{\nu}^{\mu}$ can be described by the formula (5.1) with the matrix ${ }^{37}$

$$
\alpha_{L}=\mathbf{1} \otimes \Lambda
$$

which commutes with (6.152) and, according to the general prescription (6.143), the dual realization corresponds to the matrix (cf. also (6.147))

$$
\beta_{L}=\alpha^{-1} \cdot \alpha_{L} \cdot \alpha=\alpha_{L}
$$

The last example is the space-time shift

$$
\begin{aligned}
x & \rightarrow x+b \\
\phi(x) & \rightarrow \phi(x-b),
\end{aligned}
$$

for which, according to (6.143), we get

$$
\left(x^{\prime}, \phi^{\prime}\left(x^{\prime}\right), \partial^{\prime} \phi^{\prime}\left(x^{\prime}\right)\right)=\left(x_{\alpha}+b, \phi_{\alpha}\left(x_{\alpha}\right),(\partial \phi)_{\alpha}\left(x_{\alpha}\right)\right)_{\alpha^{-1}}
$$

\footnotetext{
${ }^{37}$ Strictly speaking for scalar $\phi$ this is true only for proper Lorentz transformation with $\operatorname{det} \Lambda=1$. Other alternatives (pseudoscalar $\phi$ or improper Lorentz transformation) can be discussed analogously with minor changes.
} 
or explicitly

$$
\begin{aligned}
x^{\prime} & =x+\alpha_{B B} \operatorname{det}(\alpha)^{-1} b \\
\phi^{\prime} & =\phi-\frac{1}{2} \alpha_{B B} \alpha_{B P} \operatorname{det}(\alpha)^{-2} b^{2}+\alpha_{B P} \operatorname{det}(\alpha)^{-1} b \cdot x \\
(\partial \phi)^{\prime} & =\partial \phi+\alpha_{B P} \operatorname{det}(\alpha)^{-1} b .
\end{aligned}
$$

The dual realization of the space-time shift is therefore a composition of shift and general Galileon transformation with $\alpha$ and $b$-dependent parameters.

\subsection{Duality of the $S$ matrix}

On the quantum level the most important object is the $S$ matrix. In this section we will discuss its properties with respect to the Galileon duality and show that it is invariant with respect to the subgroups of duality transformations $\alpha_{D}(\theta)$ and $\alpha_{S}(1, \kappa)$.

Let us first briefly remind the well known equivalence theorem which makes a statement about the invariance of the $S$ matrix with respect to the field redefinitions (see e.g. [22]). The $S$ matrix can be obtained by means of LSZ formulae from the generating functional $Z[J]$ of the Green functions which can be expressed in terms of the functional integral.

$$
Z[J]=\int \mathcal{D} \phi \exp \left(\frac{\mathrm{i}}{\hbar} S[\phi]+\frac{\mathrm{i}}{\hbar}\langle J \phi\rangle\right) .
$$

In this formula we tacitly assume appropriate regularization which preserves the properties of the action with respect to the Galileon symmetry and duality transformations. The action can be expanded in powers of $\hbar$

$$
S[\phi]=\sum_{n=0}^{\infty} \hbar^{n} S_{n}[\phi] \equiv S_{0}[\phi]+S_{C T}[\phi],
$$

where $S_{0}[\phi]$ is the Galileon Lagrangian (2.4). The higher order terms $S_{n}[\phi]$ in the expansion (6.158) summed up in $S_{C T}[\phi]$ represent the counterterms which are necessary in order to renormalize the UV divergences stemming form the $n$-loop graphs. The discussion of these counterterms we postpone to the section 6.8 , here we only stress that, because of the derivative structure of the Galileon interaction vertices, the counterterms $S_{n}[\phi]$ have more derivatives per field than the basic action $S_{0}[\phi]$, and that under our assumptions on the regularization the counterterms should respect the Galileon symmetry.

In the functional integral the field $\phi$ is a dummy variable and can be freely changed by means of the field redefinition $\phi \rightarrow \mathcal{F}[\phi]$ according to

$$
Z[J]=\int \mathcal{D} \phi \operatorname{det}\left(\frac{\delta \mathcal{F}[\phi]}{\delta \phi}\right) \exp \left(\frac{\mathrm{i}}{\hbar} S[\mathcal{F}[\phi]]+\frac{\mathrm{i}}{\hbar}\langle J \mathcal{F}[\phi]\rangle\right),
$$

where we have abbreviated ${ }^{38}\langle\cdot\rangle \equiv \int \mathrm{d}^{d} x(\cdot)$. This should be compared with the generating functional $Z_{\mathcal{F}}[J]$ in the theory with the action $S_{\mathcal{F}}[\phi] \equiv S[\mathcal{F}[\phi]]$

$$
Z_{\mathcal{F}}[J]=\int \mathcal{D} \phi \exp \left(\frac{\mathrm{i}}{\hbar} S_{\mathcal{F}}[\phi]+\frac{\mathrm{i}}{\hbar}\langle J \phi\rangle\right)
$$

\footnotetext{
${ }^{38}$ In what follows we will often use this notation without further comments unless it shall lead to misinterpretation.
} 
Ignoring the Jacobian on the right hand side of (6.159) for a moment, the sufficient condition for the perturbative equivalence of the $S$ matrices in the theories with actions $S[\phi]$ and $S_{\mathcal{F}}[\phi]$ is that the Fourier transforms of the Green functions of the operators $\phi(x)$ and $\mathcal{F}[\phi](x)$ have the same one-particle poles at $p_{i}^{2}=0$ up to a simple re-scaling of the residues. ${ }^{39}$ This is achieved provided $\mathcal{F}[0]=0$ and

$$
\langle 0|\mathcal{F}[\phi](0)| p\rangle=\mathcal{Z}_{\mathcal{F}}\langle 0|\phi(0)| p\rangle
$$

with $\mathcal{Z}_{\mathcal{F}} \neq 0$. This requirement is respected by the Galileon duality transformation which are represented by the upper triangular matrices with $\alpha_{P P}=1$. To prove this, it is sufficient to investigate the dualities corresponding to $\alpha_{D}(\theta)$ and $\alpha_{S}(1, \kappa)$ separately because of the decomposition (5.27). In the former case we have

$$
\mathcal{F}[\phi](x) \equiv \phi_{\theta}(x)=\phi(x)+\theta \partial \phi(x) \cdot \partial \phi(x)+O\left(\theta^{2}, \phi^{4}\right)
$$

and therefore ${ }^{40}$

$$
\mathcal{Z}_{\mathcal{F}}=1+O(\hbar)
$$

while in the latter case we trivially ${ }^{41}$ get $\mathcal{Z}_{\mathcal{F}}=\kappa$. Thus the only obstruction which prevents us to make a statement on the equivalence of the on-shell $S$ matrices in both theories also at the loop level is the possible nontrivial functional determinant on the right hand side of (6.159). Its actual value depends on the regularization. In what follows we will show that using dimensional regularization the functional determinant equals to one for the duality transformations $\alpha_{D}(\theta)$ (the case $\alpha_{S}(1, \kappa)$ is of course trivial).

For the infinitesimal $\theta$ we can expand the functional determinant according to (6.162) as

$$
\operatorname{det}\left(\frac{\delta \phi_{\theta}(x)}{\delta \phi(y)}\right)=1+2 \theta \operatorname{Tr}(\partial \phi(x) \partial) .
$$

The trace can be further expressed in a standard way (introducing the operators $\widehat{X} \equiv x$, $\widehat{K} \equiv-\mathrm{i} \partial$ and their eigenvectors $\mid x)$ and $\mid k)$ respectively) as

$$
\begin{aligned}
\operatorname{Tr}(\partial \phi(x) \partial) & =\mathrm{i} \int \mathrm{d}^{d-2 \varepsilon} x(x|\partial \phi(\widehat{X}) \cdot \widehat{K}| x)=\mathrm{i} \int \mathrm{d}^{d-2 \varepsilon} x \mathrm{~d}^{d-2 \varepsilon} k(x|\partial \phi(\widehat{X}) \cdot \widehat{K}| k)(k \mid x) \\
& =\mathrm{i} \int \mathrm{d}^{d-2 \varepsilon} x \partial \phi(x) \cdot \int \frac{\mathrm{d}^{d-2 \varepsilon} k}{(2 \pi)^{d-2 \varepsilon}} k
\end{aligned}
$$

\footnotetext{
${ }^{39}$ Implicitly this means that the operator $\mathcal{F}[\phi](x)$ is translation invariant, i.e. $\mathcal{F}[\phi](x)=$ $e^{-i P \cdot x} \mathcal{F}[\phi](0) e^{i P \cdot x}$. Violation of this condition might prevent the applicability of the LSZ reduction formulae when passing from correlators to $S$ matrix elements.

${ }^{40}$ The $O(\hbar)$ part stems from the contributions of the terms bilinear and higher in the derivatives of the field $\phi$ on the right hand side of (6.162). These terms start to contribute to $\mathcal{Z}_{\mathcal{F}}$ only at the loop level.

${ }^{41}$ The case of $\alpha_{S}(\lambda, \kappa)$ is more complicated, because

$$
\mathcal{F}[\phi](x)=\lambda \kappa \phi\left(\lambda^{-1} x\right), \quad \int \mathrm{d}^{d} x \mathrm{e}^{\mathrm{i} p \cdot x} \lambda \kappa \phi\left(\lambda^{-1} x\right)=\lambda^{d+1} \kappa \int \mathrm{d}^{d} x \mathrm{e}^{\mathrm{i} \lambda p \cdot x} \phi(x)
$$

and the behaviour of the Green functions under the re-scaling of the momenta is governed by the renormalization group. This is the rationale for the constraint $\alpha_{P P}=1$. However, at the tree level this simplifies to scaling respecting the canonical dimensions.
} 
The first factor equals to zero for well behaved $\phi$ while the second one vanishes within the the dimensional regularization. We have thus

$$
\operatorname{det}\left(\frac{\delta \phi_{\theta}(x)}{\delta \phi(y)}\right)=1+O\left(\theta^{2}\right)
$$

For the finite transformation we can use the fact that the transformation forms a oneparametric group and thus

$$
\phi_{\theta+\xi}[\phi]=\phi_{\theta}\left[\phi_{\xi}\right]=\phi_{\xi}\left[\phi_{\theta}\right]
$$

which implies

$$
\operatorname{det}\left(\frac{\delta \phi_{\theta+\Delta \theta}}{\delta \phi_{\theta}}\right)=1+O\left((\Delta \theta)^{2}\right) .
$$

Using the formula

$$
\frac{\delta \phi_{\theta+\Delta \theta}(x)}{\delta \phi(y)}=\int \mathrm{d}^{d} z \frac{\delta \phi_{\theta+\Delta \theta}(x)}{\delta \phi_{\theta}(z)} \frac{\delta \phi_{\theta}(z)}{\delta \phi(y)}
$$

we get formally

$$
\operatorname{det}\left(\frac{\delta \phi_{\theta+\Delta \theta}}{\delta \phi}\right)=\operatorname{det}\left(\frac{\delta \phi_{\theta+\Delta \theta}}{\delta \phi_{\theta}}\right) \operatorname{det}\left(\frac{\delta \phi_{\theta}}{\delta \phi}\right)
$$

and therefore

$$
\frac{\partial}{\partial \theta} \ln \operatorname{det}\left(\frac{\delta \phi_{\theta}}{\delta \phi}\right)=0,
$$

By means of integration from 0 to $\theta$ this leads to the desired statement ${ }^{42}$

$$
\operatorname{det}\left(\frac{\delta \phi_{\theta}}{\delta \phi}\right)=1
$$

The on-shell $S$ matrices in theories with actions $S_{\mathcal{F}}[\phi]$ and $S[\phi]$ are therefore formally equivalent for the above duality transformations. This statement, however, must be taken with care. The first reason is that the counterterm part $S_{C T}[\phi]$ of the action has not the form of the Galileon Lagrangian and transforms therefore highly nontrivially with respect to the duality (note that the duality transformation is in general non-local and involves infinite number of terms, cf. appendix A). The second reason is that though we have formally established equivalence of the on-shell $S$ matrices, the off-shell Green functions stay to be different in both theories. Indeed, we have in fact only proved that Green functions of operators $\phi(x)$ in original theory and those of operators $\mathcal{F}[\phi](x)$ in the dual theory coincide. ${ }^{43}$ Therefore, the recursive construction of the counterterms in the dual

\footnotetext{
${ }^{42}$ Let us note that without the knowledge that the Jacobian of this transformation is equal one, in a standard way, we can introduced the ghost fields which would reproduce the studied determinant. At the end, however, one would find that propagators of such ghosts are proportional to 1, and thus every integration over ghost loop with momentum $l$ is of the type:

$$
\int \mathrm{d}^{d-2 \varepsilon} l \times \operatorname{Polynomial}(l)=0
$$

which is true for the dimensional regularization.

${ }^{43}$ Analogous statement can be made also for any other (composite) operator $\mathcal{O}[\phi]$. The correlators of a string of such operators within the original theory coincide with correlators of corresponding dual operators $\mathcal{O}[\mathcal{F}[\phi]]$ calculated within the dual theory. This is in accord with our discussion of the dual observables on the classical level.
} 
theory starting with the dual basic action $S_{0}[\mathcal{F}[\phi]]$ will lead to counterterm action $S_{C T}^{\mathcal{F}}[\phi]$ different from $S_{C T}[\mathcal{F}[\phi]]$. On the other hand, the counterterms from $S_{C T}[\mathcal{F}[\phi]]$ will be sufficient to cancel the divergences of the on-shell amplitudes in the dual theory. Before we proceed to the more detailed discussion of quantum correction and counterterms (we postpone it to the sections 6.8 and 6.9) we will illustrate the consequences of duality in the case of tree level scattering amplitudes.

\subsection{Tree level amplitudes}

As we have mentioned in section 2, the tree level amplitudes up to the 5pt one have surprisingly simple structure though they are sums of a large number of nontrivial contributions stemming from individual Feynman graphs with different topologies. Therefore large cancellations between different contribution have to occur the reason of which is not transparent on the Lagrangian level. In this subsection we will show on an elementary level how these results can be understood better with the help of the duality.

As we will demonstrate, the mechanism of the above mentioned cancellation is a consequence of the invariance of the $S$ matrix with respect to the duality subgroup $\alpha_{D}(\theta)$. The key observation is that the tree amplitudes in the dual theory are polynomials in the parameter $\theta$, however, due to the invariance, they are in fact $\theta$ independent. Therefore the coefficients of the above mentioned polynomial at the positive powers of $\theta$ have to vanish which gives nontrivial relations between different contributions to the amplitude.

We will also show that though the $S$ matrix is not invariant under the duality subgroup $\alpha_{S}(\lambda)$, the tree level amplitudes have simple transformation properties which can be used to relate also the $S$ matrices in theories dual with respect to $\alpha_{S}(\lambda)$.

Let us first remind some of the general properties of the tree level amplitudes. For general tree amplitudes we have

$$
I=V-1, \quad \sum_{n} n \alpha_{n}=2 I+E
$$

where $I$ and $E$ represents number of internal and external lines respectively, $V$ is number of vertices and $\alpha_{n}$ is number of vertices with $n$ legs; putting this together we get

$$
\sum_{n}\left(n \alpha_{n}-2\right)=E-2 .
$$

It is clear that any amplitude must be represented by a linear combination of the monomials $\prod_{n} d_{n}^{\alpha_{n}}$ with $d_{n}$ - independent kinematical coefficients, which carry the information on the momentum dependence of the amplitudes, explicitly ${ }^{44}$

$$
M\left(1, \ldots, E ; d_{n}\right)=\sum_{\left\{\alpha_{n}\right\}} M_{\left\{\alpha_{n}\right\}}(1, \ldots, E) \prod_{n} d_{n}^{\alpha_{n}} .
$$

Here the sum is over the sequences $\left\{\alpha_{n}\right\}_{n=3}^{d+1}$ which satisfy the condition (6.175) and the coefficients $M_{\left\{\alpha_{n}\right\}}(1, \ldots, E)$ represent the sum of Feynman diagrams with $\alpha_{n}$ vertices with

\footnotetext{
${ }^{44}$ Her we abbreviate $M\left(p_{1}, p_{2}, \ldots, p_{E} ; d_{n}\right)$ by $M\left(1, \ldots, E ; d_{n}\right)$ and similarly for the $d_{n}$ independent kinematical coefficients.
} 
$n$ legs. In what follows we restrict ourselves to case $d=4$, i.e. the sum in (6.176) is over the ordered triplets $\left\{\alpha_{3}, \alpha_{4}, \alpha_{5}\right\}$.

As we have seen in subsection 6.5 , the tree-level $S$ matrix is invariant with respect to the duality $\alpha_{D}(\theta)$, therefore the amplitudes have to satisfy the following condition

$$
\frac{\partial}{\partial \theta} M\left(1, \ldots, E ; d_{n}(\theta)\right)=0,
$$

where $d_{n}(\theta)$ are given by (5.43). This gives us non-trivial constraints on the form of the coefficients $M_{\left\{\alpha_{3}, \alpha_{4}, \alpha_{5}\right\}}(1, \ldots, E)$. Let us now study the impact of these constraint on individual amplitudes. For $E=3$ the only allowed sequence in $(6.176)$ is $\{1,0,0\}$. Inserting (5.43) into (6.176) we get

$$
M\left(1,2,3 ; d_{n}(\theta)\right)=\left(d_{3}-\frac{1}{3} \theta\right) M_{\{1,0,0\}}(1,2,3),
$$

and thus from (6.177) we get without any calculation (cf. (2.15))

$$
M_{\{1,0,0\}}(1,2,3)=0 .
$$

For $E=4$ we have in the same way

$M\left(1,2,3,4 ; d_{n}(\theta)\right)=\left(d_{3}-\frac{1}{3} \theta\right)^{2} M_{\{2,0,0\}}(1,2,3,4)+\left(d_{4}-3 \theta d_{3}+\frac{1}{2} \theta^{2}\right) M_{\{0,1,0\}}(1,2,3,4)$

and nullifying the coefficient at different powers of $\theta$ we get the constraint

$$
M_{\{2,0,0\}}(1,2,3,4)=-\frac{9}{2} M_{\{0,1,0\}}(1,2,3,4)
$$

and thus

$$
M\left(1,2,3,4 ; d_{n}\right)=\left(d_{4}-\frac{9}{2} d_{3}^{2}\right) M_{\{0,1,0\}}(1,2,3,4) .
$$

As $M_{\{0,1,0\}}(1,2,3,4)$ is just the Feynman rule for the four-point vertex (cf. (2.13)) we may immediately write

$$
M_{\{0,1,0\}}(1,2,3,4)=4 ! G(1,2,3) .
$$

Together this yields

$$
M\left(1,2,3,4 ; d_{n}\right)=12\left(2 d_{4}-9 d_{3}^{2}\right) G(1,2,3),
$$

in agreement with (2.16). We can continue with further amplitudes and find out that the duality simplifies significantly the calculation. For instance for $E=5$

$$
\begin{aligned}
M\left(1,2,3,4,5 ; d_{n}(\theta)\right)= & d_{3}^{3} M_{\{3,0,0\}}(1,2,3,4,5)+d_{3} d_{4} M_{\{1,1,0\}}(1,2,3,4,5) \\
& +d_{5} M_{\{0,0,1\}}(1,2,3,4,5)
\end{aligned}
$$

and the duality constraints are now

$$
\begin{aligned}
& M_{\{1,1,0\}}(1,2,3,4,5)=-\frac{24}{5} M_{\{0,0,1\}}(1,2,3,4,5) \\
& M_{\{3,0,0\}}(1,2,3,4,5)=\frac{72}{5} M_{\{0,0,1\}}(1,2,3,4,5) .
\end{aligned}
$$


As a consequence

$$
M\left(1,2,3,4,5 ; d_{n}\right)=\left(\frac{72}{5} d_{3}^{3}-\frac{24}{5} d_{3} d_{4}+d_{5}\right) M_{\{0,0,1\}}(1,2,3,4,5) .
$$

Again $M_{\{0,0,1\}}(1,2,3,4,5)$ is just the Feynman rule

$$
M_{\{0,0,1\}}(1,2,3,4,5)=-5 ! G(1,2,3,4)
$$

and we conclude without calculations (cf. (2.17))

$$
M\left(1,2,3,4,5 ; d_{n}\right)=-24\left(72 d_{3}^{3}-24 d_{3} d_{4}+5 d_{5}\right) G(1,2,3,4) .
$$

As a last example we take $E=6$, the computer calculation of which though possible gives rather lengthy and untransparent final output so it is difficult to reveal any regular structure hidden in it. As we will see also here the duality helps considerably.

There are four kinematical factors in this case corresponding to the sequences $\{4,0,0\}$, $\{0,2,0\},\{2,1,0\}$ and $\{1,0,1\}$. The duality constraints are

$$
\begin{aligned}
& M_{\{4,0,0\}}(1, \ldots, 6)=\frac{81}{4} M_{\{0,2,0\}}(1, \ldots, 6) \\
& M_{\{2,1,0\}}(1, \ldots, 6)=-9 M_{\{0,2,0\}}(1, \ldots, 6) \\
& M_{\{1,0,1\}}(1, \ldots, 6)=0
\end{aligned}
$$

and thus when inserted to the formula (6.176) we get finally

$$
\begin{aligned}
M\left(1, \ldots, 6 ; d_{n}\right) & =\left(\frac{81}{4} d_{3}^{4}+d_{4}^{2}-9 d_{3}^{2} d_{4}\right) M_{\{0,2,0\}}(1, \ldots, 6) \\
& =\left(d_{4}-\frac{9}{2} d_{3}^{2}\right)^{2} M_{\{0,2,0\}}(1, \ldots, 6) .
\end{aligned}
$$

Here $M_{\{0,2,0\}}$ is the sum of graphs with two four-point vertices connected by one propagator and can be therefore written in the form

$$
M_{\{0,2,0\}}(1, \ldots, 6)=-16 \sum_{\sigma \in S_{6}} \frac{G(\sigma(1), \sigma(2), \sigma(3)) G(\sigma(4), \sigma(5), \sigma(6))}{\left(p_{\sigma(1)}+p_{\sigma(2)}+p_{\sigma(3)}\right)^{2}},
$$

where we sum over the permutations of the external momenta.

Of course these results are not surprising. The tree-level $S$ matrix being an invariant of the duality subgroup $\alpha_{D}(\theta)$ can depend on $d_{n}$ only as a function of the the basic $\alpha_{D}(\theta)$ duality invariants $I_{4}, I_{5}$ given by (5.44). Because these invariants can be interpreted as $d_{4}$ and $d_{5}$ in a dual theory with $d_{3}=0$, the tree-level amplitudes must have the form

$$
M\left(1, \ldots, E ; d_{k}\right)=\sum_{\{m, n\} \geq 0} M_{\{0, m, n\}}(1, \ldots, E) I_{4}^{m} I_{5}^{n}
$$

where the summation over $m$ and $n$ must fulfil (6.175), i.e.

$$
2 m+3 n=E-2 .
$$


This general structure can be easily recognized in all the above examples. Let us note that in general case

$$
I_{4}^{m} I_{5}^{n}=\sum_{\left\{\alpha_{k}\right\}} c_{\left\{\alpha_{k}\right\}}^{m n} \prod_{k} d_{k}^{\alpha_{k}}
$$

where $c_{\left\{\alpha_{k}\right\}}^{m n}$ are rational numbers. Then comparing the coefficients (6.176) and (6.192) we get the above discussed constraints on the individual contributions to the amplitude in a general form

$$
M_{\left\{\alpha_{k}\right\}}(1, \ldots, E)=\sum_{m, n} c_{\left\{\alpha_{k}\right\}}^{m n} M_{\{0, m, n\}}(1, \ldots, E)
$$

As we have illustrated above, the tree-level amplitudes are invariants of the subgroup $\alpha_{D}(\theta)$ but also their transformation properties with respect to the scalings $\alpha_{S}(\lambda)$ and more generally $\alpha_{S}(\lambda, \kappa)$ are transparent. Let us remind that, under the $\alpha_{S}(\lambda)$ the couplings $d_{n}$ scale according its dimension (cf. (5.19) with $\Delta=(d-2) / 2$, which is the canonical dimension of the field $\phi$ )

$$
d_{n}\left(\alpha_{S}(\lambda)\right) \equiv d_{n}(\lambda)=\lambda^{-\frac{1}{2}(d+2)(n-2)} d_{n},
$$

which just corresponds to the re-scaling of the units. Note that, for $d$ even we can generalize the above scaling also to $\lambda<0$. From the homogeneity of the tree ${ }^{45}$ amplitudes

$$
\begin{aligned}
M\left(\lambda p_{1}, \ldots, \lambda p_{n} ; \lambda^{\operatorname{dim} d_{k}} d_{k}\right) & =\lambda^{\operatorname{dim} M\left(p_{1}, \ldots, p_{n} ; d_{k}\right)} M\left(p_{1}, \ldots, p_{n} ; d_{k}\right) \\
& =\lambda^{d-n \frac{d-2}{2}} M\left(p_{1}, \ldots, p_{n} ; d_{k}\right),
\end{aligned}
$$

it follows that two amplitudes with $d_{n}$ and $d_{n}(\lambda)$ are connected by

$$
M\left(p_{1}, \ldots, p_{n} ; d_{k}(\lambda)\right)=\lambda^{d-n \frac{d-2}{2}} M\left(\lambda^{-1} p_{1}, \ldots, \lambda^{-1} p_{n} ; d_{k}\right) .
$$

At tree ${ }^{46}$ level on the other hand

$$
M\left(\lambda^{-1} p_{1}, \ldots, \lambda^{-1} p_{n} ; d_{k}\right)=\lambda^{-2(n-1)} M\left(p_{1}, \ldots, p_{n} ; d_{k}\right)
$$

as we will show in the section 6.8 and therefore we get finally

$$
M\left(p_{1}, \ldots, p_{n} ; d_{k}(\lambda)\right)=\lambda^{\frac{1}{2}(d+2)(2-n)} M\left(p_{1}, \ldots, p_{n} ; d_{k}\right) .
$$

Therefore not only that it is sufficient to know the amplitudes for some representant of the group orbit of $\alpha_{D}(\theta)$ in the theory subspace $D_{d+1}^{(1)}$ but we can also travel between different (but qualitatively similar) orbits using the formula (6.200).

\footnotetext{
${ }^{45}$ Note that, at the loop level, we have additional dependence of the amplitudes on additional dimensionfull parameters, namely on the counterterm couplings as well as on the renormalization scale.

${ }^{46}$ As we will see in the subsequent sections, the loop amplitudes have higher degree of homogeneity with respect to re-scaling the momenta.
} 


\subsection{Classification of the Galileon theories}

As we have shown, some physical consequences of the Galileon theories are not directly visible from the Galileon Lagrangian. This concerns e.g. the cancellations of the various contributions to the tree-level amplitudes as well as the hidden $Z_{2}$ symmetry of the Galileon action discussed in the previous sections. However, as was seen in the latter case, such properties are usually shared by the theories which are connected by the group of duality transformations (or by some of its subgroup). It is therefore important to describe the equivalence classes of the Galileon theories with respect to the duality.

In what follows we will classify in this sense the Galileon theories in $d=3$ and 4 . We will restrict ourselves to the theory subspace $D_{d+1}^{(1)}$ with $d_{2}=1 / 4$ and $1 / 12$ respectively and we will consider only the dualities corresponding the upper triangular matrices $\alpha$ which make sense also in the quantum case. According to the results of the previous sections, such a classification is at the same time also a classification of the nontrivial tree level $S$ matrices because these are either invariants of the duality with respect to the subgroup $\alpha_{D}(\theta)$ or trivially scale with respect to $\alpha_{S}(\lambda)$.

\subsubsection{Galileons in $d=4$}

The properties of the theory which belongs to the theory subspace $D_{5}^{(1)}$ with constants $d_{3}$, $d_{4}$ and $d_{5}$ are governed by the invariants of the duality transformation $I_{4}$ and $I_{5}$ given by (5.44). Let us remind that $I_{n}$ represents the value of the constant $d_{n}$ in the theory which is dual to the original one but satisfies the condition $d_{3}=0$. We have the following cases (see figure 4):

- $I_{4}=I_{5}=0$, in this case the theory is dual to free theory

- $I_{5}=0$, in this case the theory is $Z_{2}$ invariant (it is dual to the theory with $d_{3}=d_{5}=$ $0)$. The $Z_{2}$ invariance is realized by (6.148) with $\alpha=\alpha_{D}\left(-3 d_{3}\right)$. The only non-zero amplitudes are those with even number of legs

- $I_{4}=0$, the theory is a dual to quintic Galileon (where $d_{3}=d_{4}=0$ )

- both $I_{4}, I_{5} \neq 0$, the theory is dual to $d_{3}=0, d_{4,5} \neq 0$

Let us now summarize the cases for which a concrete coupling $d_{n}$ can be removed by duality transformation. The following conditions are easily derived as the conditions for the existence of the solutions of the equations $d_{n}(\theta)=0$ with respect to $\theta$ (cf. (5.43))

- Every theory is dual to theory with $d_{3}=0$

- $I_{4}<0$, then theory is dual to just two theories with odd interactions where $d_{4}=0$ (this can be achieved by duality transformation corresponding to $\alpha_{D}\left(\theta_{ \pm}\right)$for $\theta_{ \pm}=$ $\left.3 d_{3} \pm \sqrt{-2 I_{4}}\right)$

- $I_{4}>0$, then there is no dual with $d_{4}=0$

- For $\left(8 I_{4}\right)^{3}+\left(15 I_{5}\right)^{2}>0$ theory is dual to exactly one theory with $d_{5}=0$ 


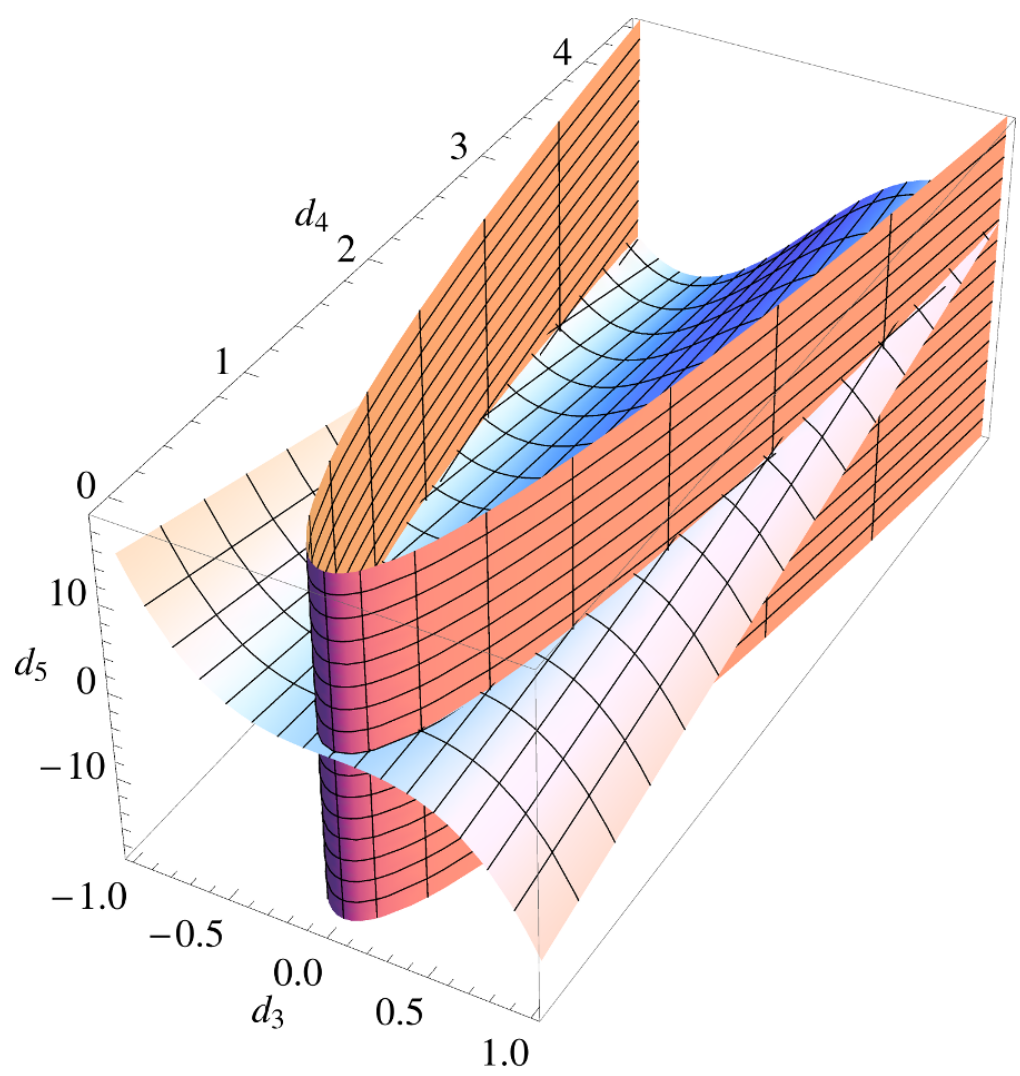

Figure 4. The surfaces $I_{4}=0$ (cylindrical one corresponding to duals of the quintic Galileon) and $I_{5}=0\left(Z_{2}\right.$ symmetric Galileons $)$ in the theory space $D_{5}^{(1)}$ with $d_{2}=1 / 12$ fixed. The intersection of these surfaces corresponds to the duals of a free theory. Both surfaces are invariant with respect to the scaling.

- For $\left(8 I_{4}\right)^{3}+\left(15 I_{5}\right)^{2}<0$ theory is dual to exactly three theories with $d_{5}=0$

The invariants $I_{4}$ and $I_{5}$ scale as $d_{4}$ and $d_{5}$, namely

$$
I_{4}(\lambda)=\lambda^{-6} I_{4}, \quad I_{5}(\lambda)=\lambda^{-9} I_{5} .
$$

Therefore by means of the scaling $\alpha_{S}(\lambda)$ we can always arrange either $I_{4}= \pm 1$ or $I_{5}=1$ (with $\lambda<0$ when necessary). To summarize, non-trivial theories (i.e. those which are not connected by dualities $\alpha_{D}(\theta)$ or $\left.\alpha_{S}(\lambda)\right)$ are

- $I_{4}=I_{5}=0$ - free theory

- $I_{4}= \pm 1, I_{5}=0-Z_{2}$ invariant theory (only even amplitudes are non-zero)

- $I_{5}=1, I_{4}=0$ - quintic Galileon

- $I_{5}=1, I_{4} \neq 0$ - general case 


\subsubsection{Galileons in $d=3$}

The situation in three dimension is even simpler. There is only one invariant of the $\alpha_{D}(\theta)$ duality

$$
I_{4}=d_{4}-\frac{9}{8} d_{3}^{2}
$$

From the previous it follows readily

$$
d_{3}(\lambda)=\lambda^{-\frac{5}{2}} d_{3}, \quad d_{4}(\lambda)=\lambda^{-5} d_{4}, \quad I_{4}(\lambda)=\lambda^{-5} I_{4}
$$

For $I_{4}<0$ we can remove $d_{4}$ by a duality $\alpha_{D}\left(\theta_{ \pm}\right)$with

$$
\theta_{ \pm}=\frac{3}{4} d_{3} \pm \frac{1}{2} \sqrt{-2 I_{4}}
$$

Provided $I_{4}>0$ there is no $\alpha_{D}(\theta)$ dual with $d_{4}(\theta)=0$ and naively there is no possibility to change the sign of $I_{4}$ by simple scaling because $\lambda<0$ is not allowed in odd dimension for theory with $d_{2 k-1} \neq 0$. However, we can first remove $d_{3}$ by $\alpha\left(3 d_{3} / 2\right)$ and only then scale with $\lambda<0$ to arrange $I_{4}=1$, because there is no odd vertex in the dual theory. This leads to the following classification

- $I_{4}=0$ - free theory

- $I_{4}=1-Z_{2}$ invariant theory

To summarize, up to the above described $\alpha_{D}(\theta)$ and $\alpha_{S}(\lambda)$ dualities there is only one non-trivial Galileon theory in three dimension the only nonzero amplitudes of which are the even ones.

\subsection{Counterterms}

From the results of the previous sections it seems possible to use the duality relations also at the quantum level. However, this is true only provided the quantum level makes sense. Starting with the basic (i.e. the tree-level) Galileon Lagrangian and choosing an appropriate regularization prescription which preserves the Galileon symmetry (in what follows we use exclusively dimensional regularization), we can construct one-loop diagrams. Such diagrams will be divergent and will thus need to be renormalized by the counterterms. From a simple dimensional consideration it is clear (and it will be explicitly shown below) that it is not possible to create such counterterms using the basic tree-level Lagrangian. We will thus have to add qualitatively new terms in the action constrained in their form only by the Galileon symmetry. In fact at any order of the loop expansion an infinite tower of new counterterms is necessary. This is of course nothing new, such a mechanism is well studied in many different effective theories e.g. in the Chiral perturbation theory (ChPT) $[44,45]$. The problem of construction of higher order Lagrangians (e.g. next-toleading-order and next-to-next-to-leading order as is the nowadays status in ChPT) is the problem by itself. Here we will merely classify the order (i.e. the degree of homogeneity in the external momenta or the number of derivatives) of the graphs and the corresponding counterterms at the given loop level. 
Let us start with the Weinberg formula [46] in $d$-dimension for the number of derivatives in the counterterm for a given graph with $L$ loops and vertices $V$ with $d_{V}$ derivatives $^{47}$

$$
D=2+(d-2) L+\sum_{V}\left(d_{V}-2\right) .
$$

The number of external legs $E$ and internal lines $I$ is connected via

$$
\sum_{V} n_{V}=2 I+E
$$

where $n_{V}$ is the number of legs for the given vertex $V$. We can also simply extract number of loops

$$
L=I-V+1
$$

Together with the previous relation this leads to

$$
E=2+\sum_{V}\left(n_{V}-2\right)-2 L
$$

and thus

$$
D-2(E-1)=(d+2) L+\sum_{V}\left(d_{V}-2\left(n_{V}-1\right)\right) .
$$

Let us now define an index of general vertex $\delta_{V}$ as a surplus of the derivatives for the general vertex in comparison with the basic Lagrangian, namely

$$
\delta_{V}=d_{V}-2\left(n_{V}-1\right)
$$

(i.e. for all the vertices of the basic Lagrangian $\delta_{V}=0$ ). In terms of such a defined index we can rewrite the formula (6.209) in the form

$$
\delta_{C T}=(d+2) L+\sum_{V} \delta_{V} \equiv \delta_{\Gamma}
$$

the right hand side of which defines the index $\delta_{\Gamma}$ of a $L$-loop graph $\Gamma$ built from the vertices with indices $\delta_{V}$. This formula is in fact an Galileon analogue of the Weinberg formula for ChPT and represents thus the connection between the loop expansion and expansion in the diagram index $\delta_{\Gamma}$, which is the order of the diagram homogeneity in momenta (modulo $\operatorname{logs}$ ) relative to tree-level diagrams constructed from the basic Lagrangian.

Note that according to the formula (6.211) each loop contributes with an additional $d+2$ term in the counterterm index $\delta_{C T}$. This means that the counterterms induced by the loops have $\delta_{C T}>0$ and therefore (because for the vertices of the basic Lagrangian $\left.\delta_{V}=0\right)$ they must be different form the terms of the basic Lagrangian. In other words the basic Galileon Lagrangian is not renormalized by loops. This proves what is often meant in the literature as the non-renormalization theorem [13-15].

\footnotetext{
${ }^{47}$ This formula holds provided the dimensional regularization or any other regularization without dimensionfull cutoff parameter is used to regulate the UV divergences. Note also that in our case of massless theory $D$ is also the superficial degree of divergence of the given graph.
} 
Let us note that similarly to the Weinberg formula for the ChPT, the formula (6.211) itself cannot be used for the proof of the generalized renormalizability. Note that the restriction $\delta_{C T}=N=$ const. constrains only the number of derivatives $d$ according to

$$
d=2(n-1)+N
$$

but it does not constrain the number $n$ of fields. In the case of ChPT the additional principle is a chiral symmetry which ensures that the infinite number of counterterms differing by the number of fields at each order combine into a finite number of chiral invariant operators. In our case we have only the Galileon symmetry at our disposal. As we have discussed above, it tells us only that the most general Galileon invariant Lagrangian is built from the building blocks $\partial_{\mu_{1}} \partial_{\mu_{2}} \ldots \partial_{\mu_{k}} \phi$ where $k \geq 2$, therefore the general counterterm with $n$ legs satisfying (6.212) has the general form

$$
\mathcal{L}_{C T}^{(n)}=\sum_{l, k_{i} \geq 2, \sum_{i} k_{i}=2(n-1)+N} c_{k_{1} k_{2} \ldots k_{n}}^{(l)} T_{(l)}^{\mu_{1}^{1} \mu_{2}^{1} \ldots \mu_{k_{1}}^{1} \ldots \mu_{1}^{n} \ldots \mu_{k_{n}}^{n}} \prod_{j=1}^{n} \partial_{\mu_{1}^{j}} \partial_{\mu_{2}^{j}} \ldots \partial_{\mu_{k_{j}}^{j}} \phi .
$$

with couplings $c_{k_{1} k_{2} \ldots k_{n}}^{(l)}$ and Lorentz invariant tensors $T_{(l)}^{\mu_{1}^{1} \mu_{2}^{1} \ldots \mu_{k_{1}}^{1} \ldots \mu_{1}^{n} \ldots \mu_{k_{n}}^{n}}$. Though for $n$ fixed we have finite number of terms, the number $n$ increases to infinity and already at the one loop level (where $N=d+2$ ) we get infinite number of independent terms.

Let us note that quantum Galileon is in principal a two scale theory. At the classical level there is a scale (let us denote it $F$ ), which is responsible for the hierarchy of the nonlinearities in the basic classical Lagrangian (2.4) [4]. Restoring the correct dimensions of the tree-level Galileon couplings we can write for the general term of the basic Lagrangian schematically (up to $O(1)$ dimensionless constant, cf. (2.8))

$$
\mathcal{L}_{b}^{(n)} \sim(\partial \phi)^{2}\left(\frac{\partial \partial \phi}{F^{d_{\phi}+2}}\right)^{n-2} \sim F^{-\frac{(d+2)(n-2)}{2}}
$$

where $d_{\phi}=(d-2) / 2$ is the canonical dimension of the Galileon field. On the other hand, according to the formula (6.211), the quantum corrections can be organized as a expansion in powers of the characteristic quantum scale, let us denote it $\Lambda$. Each counterterm with index $\delta_{C T}$ is suppressed by a power $\Lambda^{-\delta_{C T}}$. Schematically (up to $O(1)$ dimensionless coupling constant, cf. (6.213))

$$
\mathcal{L}_{C T}^{\left(n, \delta_{C T}\right)} \sim F^{d}\left(\frac{F}{\Lambda}\right)^{2} \prod_{j=1}^{n}\left(\frac{\partial}{\Lambda}\right)^{k_{i}}\left(\frac{\partial \partial \phi}{F^{d_{\phi}+2}}\right) \sim F^{-\frac{(d+2)(n-2)}{2}} \Lambda^{-\delta_{C T}},
$$

where we have assumed the constraint $\sum_{i}\left(k_{i}+2\right)=2(n-1)+\delta_{C T}$. Note that we have to restore the correct dimension of $\mathcal{L}_{C T}^{\left(n, \delta_{C T}\right)}$ using the scale $F$ in order not to disturb the hierarchy of the counterterms.

The requirement of consistency of the Galileon as an effective theory at the quantum level puts however constraint on the above two scales. The point is that the renormalized loop graphs and the corresponding counterterms contributions have to be numerically of the same order. To get this constraint let us assume a general graph $\Gamma$ with $L$ loops, $E$ 
external legs and $V$ vertices (each vertex has index $\delta_{V}$ and $n_{V}$ legs). Its contribution is schematically ${ }^{48}$

$$
\Gamma \sim\left(\frac{1}{4 \pi}\right)^{2 L} \frac{1}{\Lambda^{\Delta_{\Gamma}^{\Lambda}}} \frac{1}{F^{\Delta_{\Gamma}^{F}}}
$$

where

$$
\Delta_{\Gamma}^{\Lambda}=\sum_{V} \delta_{V}, \quad \Delta_{\Gamma}^{F}=\sum_{V} \frac{(d+2)}{2}\left(n_{V}-2\right)=\frac{(d+2)}{2}(2 L+E-2)
$$

and we have used (6.208). For the counterterm contribution we get

$$
C T \sim \frac{1}{\Lambda^{\delta_{C T}}} \frac{1}{F^{\frac{(d+2)(E-2)}{2}}},
$$

where $\delta_{C T}$ is given by (6.211). Requiring $\Gamma \sim C T$ gives then the desired relation between the classical and quantum scales

$$
\Lambda \sim(4 \pi)^{\frac{2}{d+2}} F
$$

or $\Lambda \sim 2.3 F$ for $d=4$; both scales are therefore forced to be roughly of the same order of magnitude in this case. This is analogue of the formula known in ChPT which relates the pion decay constant with the scale of the chiral symmetry breaking

$$
\Lambda_{\chi P T} \sim(4 \pi)^{\frac{2}{d-2}} F_{\pi}
$$

which however for $d=4$ requires $\Lambda_{\chi P T}$ to be one order of magnitude larger than $F_{\pi}$.

\subsection{Examples of one-loop order duality}

In the previous sections we have explicitly calculated the tree-level scattering amplitudes of the Galileon fields up to six particles. The non-trivial results start with the four-point scattering. In this section we will focus on this process and will study it at one-loop order. Of course, as mentioned above, such a full calculation would necessary need inclusion of so-far undefined Lagrangian $\mathcal{L}_{C T}^{(4)}$, which would play a role of counterterms in this process. However, our main motivation is to explicitly show that the duality is not spoiled at the quantum level (i.e. by loop contributions) at least for the graphs with the vertices from the basic Galileon Lagrangian. We will thus first calculate dimensionally regularized individual contributions to 4-pt scattering at one-loop order in one Galileon theory and show that the final result is related to other Galileon theory connected by duality.

In the table 1 we summarize the one-loop diagrams to be calculated and their corresponding divergent parts in $d=4$ dimension (the full results in $d$ dimension for $A_{1-6}$ are summarized in appendix G). Here we have used the standard Mandelstam variables for four-point scattering:

$$
s=\left(p_{1}+p_{2}\right)^{2}, \quad t=\left(p_{1}+p_{3}\right)^{2}, \quad u=\left(p_{1}+p_{4}\right)^{2}
$$

\footnotetext{
${ }^{48}$ Here the factor $(4 \pi)^{-2}$ is generic for each loop momentum integration.
} 


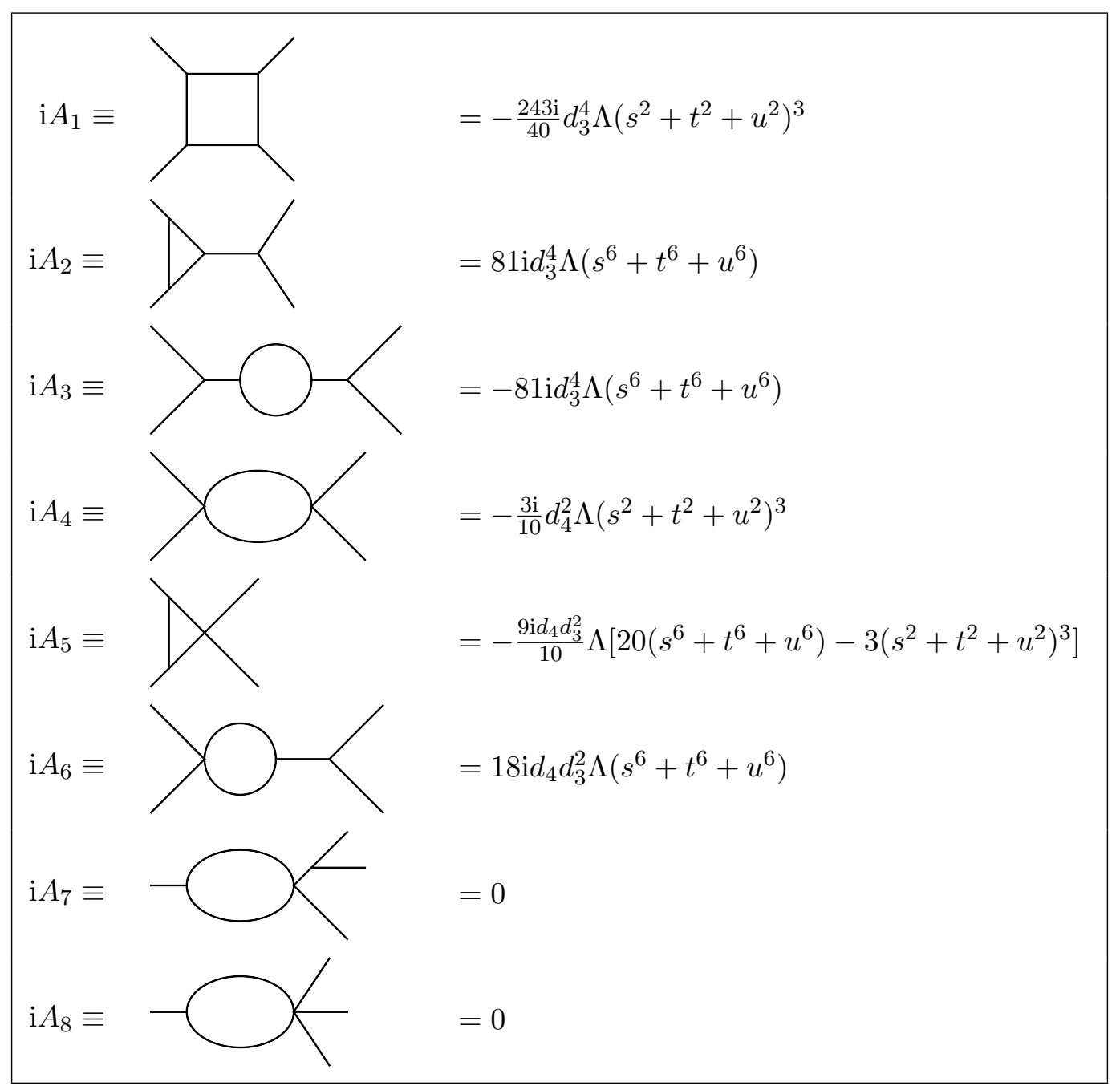

Table 1. One loop graphs contributing to the 4pt amplitude and their divergent parts.

where all momenta are ingoing and on-shell so that $s+t+u=0$. The singularity in $d=4$ dimension is given by

$$
\Lambda=\frac{1}{(4 \pi)^{2}} \frac{1}{d-4}
$$

Due to specific form of the of 3-pt vertex in the Galileon theory which can be rewritten in the form

$$
\mathcal{V}_{3}\left(q_{1}, q_{2}, q_{3}\right)=6 d_{3}\left[\left(q_{1} \cdot q_{2}\right) q_{3}^{2}+\left(q_{1} \cdot q_{3}\right) p_{2}^{2}+\left(q_{2} \cdot q_{3}\right) p_{1}^{2}\right]
$$

the contributions $A_{7}$ and $A_{8}$ (corresponding to graphs for which $\mathcal{V}_{3}$ is one of the two vertices of a bubble) are zero also for general $d$. Indeed, with external momenta on shell, the only term of $(6.222)$ which could contribute is schematically $\left(p_{\text {ext }} \cdot l\right)\left(p_{\text {ext }}-l\right)^{2}$ where $p_{\text {ext }}$ is one of the external momenta and $l$ is the loop momentum. Therefore the $\left(p_{\text {ext }}-l\right)^{2}$ factor cancels one of the bubble propagators which thus degenerate in a massless tadpole and the latter is zero in dimensional regularization. 


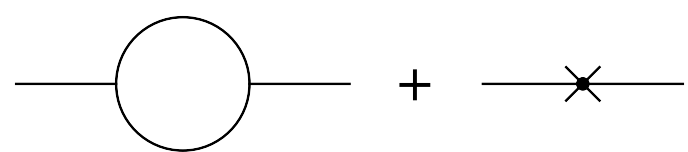

Figure 5. The Feynman graph for the Galileon self-energy and its counterterm.

Summing the diagrams together, we get that the divergent part of the amplitude for the 4-pt galileon-scattering at the one-loop order is

$$
A^{\mathrm{div}}=\sum_{i} A_{i}^{\mathrm{div}}=-\frac{3}{40} \Lambda\left(9 d_{3}^{2}-2 d_{4}\right)^{2}\left(s^{2}+t^{2}+u^{2}\right)^{3}=-\frac{3}{10} \Lambda I_{4}^{2}\left(s^{2}+t^{2}+u^{2}\right)^{3} .
$$

Note that the degree of homogeneity in external momenta is in accord with the formula (6.211). As we have expected, the singular part (and in fact also the complete result (G.8), cf. appendix G) depend on the $\alpha_{D}(\theta)$ duality invariant $I_{4}$ which illustrates the conclusions of section 6.5 that $\alpha_{D}(\theta)$ dual theories produce the same $S$-matrices. This offers also another possibility how to use the duality relations similar to that we have discussed for the tree amplitudes in section 6.6. Because $I_{4}$ is the coupling $d_{4}$ in the dual Galileon theory with new constants $d_{i}\left(\theta^{*}\right)$ such as $d_{3}\left(\theta^{*}\right)=0$ we can effectively eliminate 3 pt vertices by passing to this dual theory. The only diagram which is left to calculate in such a dual theory is $A_{4}$ which simplifies the calculations considerably.

Let us present another simple example of the one-loop calculation concerning the selfenergy correction for the Galileon field. The relevant graph is depicted in figure 5 and the explicit result for the divergent part reads (see appendix $\mathrm{G}$ for the complete result)

$$
\Sigma^{\operatorname{div}}(p)=9 \Lambda d_{3}^{4}\left(p^{2}\right)^{4},
$$

cf. also [47]. Therefore, on one hand, in Galileon theories with $d_{3} \neq 0$ we need a counterterm $\mathcal{L}_{C T}^{(2)}$ of the general form (6.213) to renormalize this divergence. The corresponding Feynman rule reads

$$
\mathcal{V}_{2}\left(p_{1}\right)=\mu^{d-4}\left(-9 \Lambda d_{3}^{4}+C^{r}(\mu)\right)\left(p^{2}\right)^{4},
$$

where $\mu$ is the dimensional regularization scale which is necessary to restore the canonical dimension of the loop integration and where $C^{r}(\mu)$ is a linear combination of the finite parts of the counterterm couplings $c_{k_{1} k_{2}}^{(l)}$ in (6.213) renormalized at scale $\mu$. On the other hand, in the above mentioned dual theory with $d_{3}\left(\theta^{*}\right)=0$ such a divergence does not occur. This is consequence of the fact that off-shell Green functions are not invariants with respect to the duality as discussed in section 6.5.

Extreme example of such non-invariance of the counterterms is the case of the free theory and some of its $\alpha_{D}(\theta)$ duals. While the free theory does not need any counterterm of the above type, its dual always ${ }^{49}$ does. However, as far as the $S$ matrix is concerned, no counterterms are needed for the graphs with the vertices from the basic dual Lagrangian because these graphs have to combine into the trivial $S$ matrix of the original free theory which is trivially divergence-free. Therefore, the contribution of the divergent part

\footnotetext{
${ }^{49}$ Note that any such dual has $d_{3}=-\theta / 3 \neq 0$.
} 
of (6.225) and analogical counterterms (which are needed to renormalize the divergent subgraphs in the table 1) has to cancel in the final result. This is, however, not true for the finite part of the counterterms the couplings of which are in principle independent. E.g. the renormalization of the bubble subgraph in the graphs $A_{3}$ in table 1 brings about the contribution (for $d \rightarrow 4$ )

$$
A_{3}^{C T}=-9 \mathrm{i} C^{r}(\mu)\left(s^{6}+t^{6}+u^{6}\right) .
$$

Therefore, the only possibility how to recover the free theory $S$ matrix also in the dual theory with counterterms is to set at some scale all the renormalized counterterm coupling constants equal to zero. Because the couplings run with the renormalization scale, this might seem to be insufficient, because at another scale the finite parts of the counterterms are in general nonzero. However, because in the amplitude all the contributions of the divergent parts of the counterterms cancel, in the same way are also cancelled all the contributions stemming from the changes of the counterterms couplings with change of the renormalization scale. ${ }^{50}$

\section{Summary}

In this paper we have studied the duality transformations of the general Galileon theories in $d$ dimensions. First we have reviewed the interpretation of the Galileon as a Goldstone boson of the spontaneous symmetry breakdown according to the pattern $G A L(d, 1) \rightarrow$ $I S O(d-1,1)$ and the identification of its action as the generalized WZW term. Then we have studied the most general coordinate transformations on the corresponding coset space $G A L(d, 1) / S O(d-1,1)$. The requirement that such a general transformation acts linearly on the basic building blocks of the Galileon Lagrangian (and therefore it represents a duality transformation) constraints the form of the transformation uniquely up to four free parameters. Under composition these duality transformations form a group which can be identified with $G L(2, \mathbf{R})$. The explicit form of the duality transformation for general $\alpha \equiv\left\{\alpha_{i j}\right\}_{i, j=1}^{2} \in G L(2, \mathbf{R})$ reads

$$
\begin{aligned}
x_{\alpha}= & \alpha_{11} x+\alpha_{12} \partial \phi(x) \\
\phi_{\alpha}\left(x_{\alpha}\right)= & \operatorname{det}(\alpha) \phi(x) \\
& +\frac{1}{2}\left(\alpha_{12} \alpha_{22} \partial \phi(x) \cdot \partial \phi(x)+2 \alpha_{12} \alpha_{21} x \cdot \partial \phi(x)+\alpha_{11} \alpha_{21} x^{2}\right) .
\end{aligned}
$$

All the up to now known Galileon dualities can be identified as special elements (or oneparametric subgroups) of this duality group. We have also studied its action on the space of the Galileon theories and found a basis of the independent invariants of one of its most interesting one-parametric subgroups

$$
\alpha_{D}(\theta)=\left(\begin{array}{cc}
1 & -2 \theta \\
0 & 1
\end{array}\right)
$$

\footnotetext{
${ }^{50}$ Note that within dimensional regularization, the coefficient at the $\ln \left(\mu / \mu^{\prime}\right)$ term in the formula for the running of the renormalized one-loop coupling coincide with the coefficient at the $\Lambda$ in formula for the bare coupling.
} 
This subgroup is represented in the space of fields as a field redefinition which can be understood both as a simultaneous space-time coordinates and field transformation or as a non-local change of the fields which includes infinite number of derivative dependent terms.

We have then studied the applications of the duality group. We have shown that we can relate the classical covariant phase spaces of dual theories and enlarge the duality transformation to the classical observables. In order to avoid apparent paradoxes, correct dual observables within the dual theory have to be used when we want to get the results of the original theory.

The duality of phase spaces can be used to generate classical solution of the interacting Galileon theory from the solution of the more simple one even when the Galileon is coupled to the local external source. We have studied two such sources, namely the point-like and string-like ones. Here the duality appears to be an efficient tool because of the symmetries which effectively reduce the dimensionality of the problems.

We have also discussed the fluctuations of the classical solutions in the linearized approximation. We have found duality transformation of the corresponding classical covariant phase spaces and corresponding observables and discussed the geometrical aspects of the problem with superluminal propagation of fluctuations. The general consideration has been illustrated by two explicit examples, namely the fluctuations of the plane wave and cylindrically symmetric classical solutions.

We have also established the dual formulation of the additional symmetries of the Lagrangian. We have shown that these symmetries might be hidden within the dual theory and found e.g. that the $Z_{2}$ symmetry and space time translations are realized non-linearly and non-locally.

Then we have discussed the transformation properties of the $S$ matrix and established its formal invariance within the dimensional regularization, though only the tree-level (classical) part of the complete action transforms nicely under the duality field redefinition. As a next issue we have demonstrated the usefulness of the $S$ matrix duality for calculations of the tree on-shell scattering amplitudes and for finding the relations between the contributions of the apparently very different Feynman graphs with completely different topologies.

As another example we have classified the equivalence classes (with respect to the duality subgroup $\alpha_{D}(\theta)$ combined with scaling) of the Galileon theories (and at the same time of the tree-level $S$ matrices) in three and four dimensions. We found e.g. that there is up to the above dualities only one nontrivial interacting theory in three dimensions which exhibits the $Z_{2}$ symmetry. Then we have discussed the transformation properties of the $S$ matrix on the loop level. As we have discussed on a concrete example of the oneloop four-point on-shell amplitude, due to the counterterms the duality is not completely straightforward. It rather holds on the regularized level for the loop graphs with vertices from the basic tree-level Lagrangian. We have also touched the problem of the counterterms classification based on a generalization of the Weinberg formula and with help of the latter we discussed the non-renormalization theorem. 
Note added. After this work was completed two works [40, 38] closely connected with the topic studied in this paper appeared. Both these papers concern the properties of the one-parametric duality subgroup denoted as $\alpha_{D}(\theta)$ in our notation and partially overlap with our results.

\section{Acknowledgments}

We would like to thank Nima Arkani-Hamed and Jaroslav Trnka for turning our attention to Galileon theory and Jaroslav Trnka and Jiří Hořejší for useful discussions and comments on the manuscript. This work is supported by Charles University in Prague, project PRVOUK P45 and by Ministry of Education of the Czech Republic, grants LG 13031 and LH 14035.

\section{A Bottom up construction of the duality subgroup $\alpha_{D}(\theta)$}

In this appendix we get more elementary treatment of the Galileon duality corresponding to the subgroup $\alpha_{D}(\theta)$. In fact this was the way we had started to think about the Galileon duality.

Let us assume an infinitesimal field transformation

$$
\phi \rightarrow \phi+\theta \partial \phi \cdot \partial \phi
$$

where $\theta$ infinitesimal parameter. The infinitesimal change of $\phi$ can be also understood as an action of the following operator (which is defined on the space of the functionals $F[\phi]$ of the field $\phi$ )

$$
\delta_{\theta} \equiv \theta\left\langle\delta \phi \frac{\delta}{\delta \phi}\right\rangle=\theta\left\langle\partial \phi \cdot \partial \phi \frac{\delta}{\delta \phi}\right\rangle
$$

on a special functional $F[\phi](x)=\phi(x)$. Here and in what follows we again abbreviate $\langle\cdot\rangle \equiv \int \mathrm{d}^{d} x(\cdot)$.

Acting by the operator $\delta_{\theta}$ on the Galileon action (cf. (2.4))

$$
S[\phi]=\sum_{n=1}^{d+1} d_{n}\left\langle\phi \mathcal{L}_{n-1}^{\mathrm{der}}\right\rangle
$$

we get

$$
\delta_{\theta} S[\phi]=\theta\left\langle\partial \phi \cdot \partial \phi \frac{\delta S[\phi]}{\delta \phi}\right\rangle=\sum_{n=2}^{d+1} \theta n d_{n}\left\langle\partial \phi \cdot \partial \phi \mathcal{L}_{n-1}^{\mathrm{der}}\right\rangle
$$

which can be rewritten with help of the formula (2.12) to the form

$$
\delta_{\theta} S[\phi]=-2 \sum_{n=3}^{d+1} \theta \frac{n-1}{n}(d-n+2) d_{n-1}\left\langle\phi \mathcal{L}_{n-1}^{\mathrm{der}}\right\rangle=\sum_{n=3}^{d+1} \theta \delta d_{n}\left\langle\phi \mathcal{L}_{n-1}^{\mathrm{der}}\right\rangle
$$

with

$$
\delta d_{n}=-2 \frac{n-1}{n}(d-n+2) d_{n-1}
$$


Therefore to the first order in $\theta$ the transformation (A.1) conserves the Galileon structure of the Lagrangian and merely shifts the coupling constants $d_{n}$ by $\delta d_{n}$. Note that the transformation (A.1) with finite $\theta$ can be used to eliminate the cubic term from the interaction Lagrangian, however, the Galileon structure of the Lagrangian is spoiled with additional interaction terms which are generated by the transformation. The way how to eliminate the cubic term consistently without leaving the space of the Galileon theories is now clear. It suffices to construct the finite transformation by means of iteration of the infinitesimal one, i.e. to exponentialize it according to

$$
\phi_{\theta}=\exp \left(\delta_{\theta}\right) \phi=\exp \left\langle\theta \partial \phi \cdot \partial \phi \frac{\delta}{\delta \phi}\right\rangle \phi=\phi+\theta \partial \phi \cdot \partial \phi+2 \theta^{2} \partial \phi \cdot \partial \partial \phi \cdot \partial \phi+\ldots
$$

Applying this finite transformation to the Galileon action results in a dual action $S_{\theta}[\phi]$ defined as

$$
S_{\theta}[\phi] \equiv S\left[\phi_{\theta}\right]=\exp \delta_{\theta} S[\phi]=\sum_{n=2}^{d+1} d_{n}(\theta)\left\langle\phi \mathcal{L}_{n-1}^{\mathrm{der}}\right\rangle .
$$

It is not difficult to show that $d_{n}(\theta)=d_{n}\left(\alpha_{D}(\theta)\right)$ where the right hand side is given by (5.26). From this construction it is clear that the transformations $\phi_{\theta}$ form a one parametric group.

In what follows we will give an alternative elementary derivation of the explicit form of $\phi_{\theta}$. Let us denote

$$
\phi(\theta, x)=\exp \left\langle\theta \partial \phi \cdot \partial \phi \frac{\delta}{\delta \phi}\right\rangle \phi(x)
$$

then we get by derivative with respect to $\theta$

$$
\begin{aligned}
\frac{\partial \phi(\theta, x)}{\partial \theta} & =\exp \left\langle\theta \partial \phi \cdot \partial \phi \frac{\delta}{\delta \phi}\right\rangle\left\langle\partial \phi \cdot \partial \phi \frac{\delta}{\delta \phi}\right\rangle \phi(x) \\
& =\exp \left\langle\theta \partial \phi \cdot \partial \phi \frac{\delta}{\delta \phi}\right\rangle \partial_{\mu} \phi(\theta, x) \partial^{\mu} \phi(\theta, x)=\partial_{\mu} \phi(\theta, x) \partial^{\mu} \phi(\theta, x) .
\end{aligned}
$$

Therefore the function $\phi(\theta, x)$ is a solution of the following Cauchy problem for the partial differential equation of the first order

$$
\frac{\partial \phi(\theta, x)}{\partial \theta}=\partial_{\mu} \phi(\theta, x) \partial^{\mu} \phi(\theta, x), \quad \phi(0, x)=\phi(x) .
$$

This problem can be solved by standard method of characteristics which are the solutions of a set of ordinary differential equations

$$
\frac{\mathrm{d} \theta}{\mathrm{d} t}=1, \quad \frac{\mathrm{d} P}{\mathrm{~d} t}=0, \quad \frac{\mathrm{d} x}{\mathrm{~d} t}=-2 p, \quad \frac{\mathrm{d} p}{\mathrm{~d} t}=0, \quad \frac{\mathrm{d} \phi}{\mathrm{d} t}=-2 p \cdot p+P^{2} .
$$

The solution of these equation is

$$
\theta=\theta_{0}+t, \quad P=P_{0}, \quad p=p_{0}, \quad x=x_{0}-2 t p_{0}, \quad \phi=\phi_{0}+t\left(P_{0}^{2}-2 p_{0} \cdot p_{0}\right) .
$$

The general recipe how to get the $d+1$-dimensional integral surface corresponding to the equation (A.10) consist of two steps. First we replace the integrations constants $\theta_{0}, \ldots, \phi_{0}$ 
with functions of $d$ parameters $a_{i}, i=1, \ldots, d$ in such a way that the following conditions are satisfied

$$
P_{0}\left(a_{i}\right)-p_{0}\left(a_{i}\right) \cdot p_{0}\left(a_{i}\right)=0, \quad \mathrm{~d} \phi_{0}\left(a_{i}\right)=p_{0}\left(a_{i}\right) \cdot \mathrm{d} x_{0}\left(a_{i}\right)+P_{0}\left(a_{i}\right) \mathrm{d} \theta_{0}\left(a_{i}\right)
$$

and subsequently we eliminate the parameters $a_{i}$ and $t$ from the equations

$$
\begin{aligned}
& \theta=\theta_{0}\left(a_{i}\right)+t, \quad x=x_{0}\left(a_{i}\right)-2 t p_{0}\left(a_{i}\right) \\
& \phi=\phi_{0}\left(a_{i}\right)+t\left(P_{0}^{2}\left(a_{i}\right)-2 p_{0}\left(a_{i}\right) \cdot p_{0}\left(a_{i}\right)\right) .
\end{aligned}
$$

Let us choose the parameters $a_{i}$ to be just $x_{0}$, we get then

$$
t=\theta-\theta_{0}\left(x_{0}\right), \quad P_{0}\left(x_{0}\right)=p_{0}\left(x_{0}\right) \cdot p_{0}\left(x_{0}\right), \quad p_{0}\left(x_{0}\right)=\partial \phi_{0}\left(x_{0}\right)-P_{0}\left(x_{0}\right) \partial \theta_{0}\left(x_{0}\right),
$$

and

$$
\begin{aligned}
x & =x_{0}-2 t p_{0}\left(x_{0}\right) \\
& =x_{0}-2\left(\theta-\theta_{0}\left(x_{0}\right)\right)\left(\partial \phi_{0}\left(x_{0}\right)-p_{0}\left(x_{0}\right) \cdot p_{0}\left(x_{0}\right) \partial \theta_{0}\left(x_{0}\right)\right) \\
\phi & =\phi_{0}\left(x_{0}\right)+t\left(P_{0}^{2}\left(x_{0}\right)-2 p_{0}\left(x_{0}\right) \cdot p_{0}\left(x_{0}\right)\right) \\
& =\phi_{0}\left(x_{0}\right)-\left(\theta-\theta_{0}\left(x_{0}\right)\right) p_{0}\left(x_{0}\right) \cdot p_{0}\left(x_{0}\right) .
\end{aligned}
$$

A special choice $\theta_{0}\left(x_{0}\right)=0$ gives

$$
x=x_{0}-2 \theta \partial \phi_{0}\left(x_{0}\right), \quad \phi(\theta, x)=\phi_{0}\left(x_{0}\right)-\theta \partial \phi_{0}\left(x_{0}\right) \cdot \partial \phi_{0}\left(x_{0}\right) .
$$

The initial condition of the Cauchy problem is $\phi(0, x)=\phi(x)$ and therefore $\phi_{0}\left(x_{0}\right)=\phi\left(x_{0}\right)$. Thus the final solution of the Cauchy problem is

$$
x=x_{0}-2 \theta \partial \phi\left(x_{0}\right), \quad \phi(\theta, x)=\phi\left(x_{0}\right)-\theta \partial \phi\left(x_{0}\right) \cdot \partial \phi\left(x_{0}\right)
$$

which is nothing else but the duality transformation (5.25).

\section{B Compatibility of duality and IHC constraint}

In this appendix we demonstrate by explicit calculation the consistency of the duality transformation with the IHC constraint. For the derivative of the field $\phi$ with respect to the unprimed coordinates we get with help of the second row of (4.19)

$$
\begin{aligned}
\partial \phi= & \partial x^{\prime} \cdot \partial^{\prime} \phi \\
= & \partial x^{\prime} \cdot\left[\operatorname{det}\left(\alpha_{I J}\right) \partial^{\prime} \phi^{\prime}\right. \\
& \left.+\frac{1}{2}\left(2 \alpha_{P B} \alpha_{B B} \partial^{\prime} \partial^{\prime} \phi^{\prime} \cdot \partial^{\prime} \phi^{\prime}+2 \alpha_{P B} \alpha_{B P} \partial^{\prime} \phi^{\prime}+2 \alpha_{P B} \alpha_{B P} x^{\prime} \cdot \partial^{\prime} \partial^{\prime} \phi^{\prime}+2 \alpha_{P P} \alpha_{B P} x^{\prime}\right)\right] .
\end{aligned}
$$

Differentiation of the first row of (4.19) we get

$$
\eta=\partial x^{\prime} \cdot\left(\alpha_{P P} \eta+\alpha_{P B} \partial^{\prime} \partial^{\prime} \phi^{\prime}\right)
$$


thus

$$
\begin{aligned}
\partial \phi= & \operatorname{det}\left(\alpha_{I J}\right) \partial x^{\prime} \cdot \partial^{\prime} \phi^{\prime}+\alpha_{B B} \alpha_{P B} \partial x^{\prime} \cdot \partial^{\prime} \partial^{\prime} \phi^{\prime} \cdot \partial^{\prime} \phi^{\prime} \\
& +\alpha_{P B} \alpha_{B P} \partial x^{\prime} \cdot \partial^{\prime} \phi^{\prime}+\alpha_{P B} \alpha_{B P} \partial x^{\prime} \cdot \partial^{\prime} \partial^{\prime} \phi^{\prime} \cdot x^{\prime}+\alpha_{P P} \alpha_{B P} \partial x^{\prime} \cdot x^{\prime} \\
= & \operatorname{det}\left(\alpha_{I J}\right) \partial x^{\prime} \cdot \partial^{\prime} \phi^{\prime}+\alpha_{B B}\left(\eta-\alpha_{P P} \partial x^{\prime}\right) \cdot \partial^{\prime} \phi^{\prime}+\alpha_{P B} \alpha_{B P} \partial x^{\prime} \cdot \partial^{\prime} \phi^{\prime} \\
& +\alpha_{B P}\left(\eta-\alpha_{P P} \partial x^{\prime}\right) \cdot x^{\prime}+\alpha_{P P} \alpha_{B P} \partial x^{\prime} \cdot x^{\prime} \\
= & \operatorname{det}\left(\alpha_{I J}\right) \partial x^{\prime} \cdot \partial^{\prime} \phi^{\prime}+\alpha_{B B} \partial^{\prime} \phi^{\prime}-\left(\alpha_{B B} \alpha_{P P}-\alpha_{P B} \alpha_{B P}\right) \partial x^{\prime} \cdot \partial^{\prime} \phi^{\prime}+\alpha_{B P} x^{\prime} \\
= & \alpha_{B B} \partial^{\prime} \phi^{\prime}+\alpha_{B P} x^{\prime}
\end{aligned}
$$

where we have used the integrability constraint (4.13) in the last line.

\section{Remark on dual observables in the linearized fluctuation theories}

In section 6.3 we have shown that linearized actions for fluctuations satisfy

$$
S_{\theta}\left[\phi_{*}, \chi\right]=S\left[\left(\phi_{*}\right)_{\theta}, \chi_{\theta}\right]
$$

where

$$
\begin{aligned}
S_{\theta}\left[\phi_{*}, \chi\right] & =\left.\frac{1}{2} \int \mathrm{d}^{d} x \mathrm{~d}^{d} y \chi(x) \frac{\delta^{2} S_{\theta}[\phi]}{\delta \phi(x) \delta \phi(y)}\right|_{\phi_{*}} \chi(y) \\
S\left[\left(\phi_{*}\right)_{\theta}, \chi\right] & =\left.\frac{1}{2} \int \mathrm{d}^{d} x \mathrm{~d}^{d} y \chi(x) \frac{\delta^{2} S[\phi]}{\delta \phi(x) \delta \phi(y)}\right|_{\left(\phi_{*}\right)_{\theta}} \chi(y)
\end{aligned}
$$

This relation enabled us to relate the solutions for the fluctuations in both theories and e.g. to prove, that the general theory might show superluminal propagation of the fluctuations even though it corresponds to a dual theory which is healthy. Here we will show opposite, i.e. that apparently healthy theory might show superluminal propagation of the fluctuations of some properly chosen operators. Let us add to $S\left[\left(\phi_{*}\right)_{\theta}, \chi_{\theta}\right]$ a source term

$$
S_{J}\left[\chi_{\theta}\right]=\int \mathrm{d}^{d} x J(x) \chi_{\theta}(x) .
$$

After some manipulations we get

$$
S_{J}\left[\chi_{\theta}\right]=\int \mathrm{d}^{d} x J(x) \chi\left(X\left[\phi_{*}\right](x)\right)
$$

Therefore we have

$$
S_{\theta}\left[\phi_{*}, \chi\right]+\int \mathrm{d}^{d} x J(x) \chi\left(X\left[\phi_{*}\right](x)\right)=S\left[\left(\phi_{*}\right)_{\theta}, \chi_{\theta}\right]+\int \mathrm{d}^{d} x J(x) \chi_{\theta}(x) .
$$

Suppose, that we have chosen $\theta$ in such a way that the $S_{\theta}\left[\phi_{*}, \chi\right]$ is healthy (e.g. $d_{3}(\theta)=0$ and the background is a plane wave). Therefore, in the framework of such a healthy theory the nonlocal operator $\chi\left(X\left[\phi_{*}\right](x)\right)$ has the same superluminal propagation as the perturbations $\chi(x)$ in the theory with action $S\left[\left(\phi_{*}\right)_{\theta}, \chi\right]$. Indeed, the generating functional of the correlators of the operators $\chi\left(X\left[\phi_{*}\right](x)\right)$ in the healthy theory

$$
Z_{\theta}\left[J, \phi_{*}\right]=\int \mathcal{D} \chi \exp \left(\frac{\mathrm{i}}{\hbar} S_{\theta}\left[\phi_{*}, \chi\right]+\frac{\mathrm{i}}{\hbar} \int \mathrm{d}^{d} x J(x) \chi\left(X\left[\phi_{*}\right](x)\right)\right)
$$


can be obtained form the generating functional for the perturbations $\chi$ in the pathological theory by means of change of variables,

$$
\begin{aligned}
Z\left[J,\left(\phi_{*}\right)_{\theta}\right] & =\int \mathcal{D} \chi \exp \left(\frac{\mathrm{i}}{\hbar} S\left[\left(\phi_{*}\right)_{\theta}, \chi\right]+\frac{\mathrm{i}}{\hbar} \int \mathrm{d}^{d} x J(x) \chi(x)\right) \\
& =\int \mathcal{D} \chi \operatorname{det}\left(\frac{\delta \chi_{\theta}}{\delta \chi}\right) \exp \left(\frac{\mathrm{i}}{\hbar} S_{\theta}\left[\phi_{*}, \chi\right]+\frac{\mathrm{i}}{\hbar} \int \mathrm{d}^{d} x J(x) \chi\left(X\left[\phi_{*}\right](x)\right)\right) \\
& =Z_{\theta}\left[J, \phi_{*}\right] .
\end{aligned}
$$

Here we have used

$$
\frac{\delta \chi_{\theta}(x)}{\delta \chi(y)}=\frac{\delta \chi\left(X\left[\phi_{*}\right](x)\right)}{\delta \chi(y)}=\delta^{(d)}\left(X\left[\phi_{*}\right](x)-y\right)
$$

and thus $\operatorname{det}\left(\delta \chi_{\theta} / \delta \chi\right)=1$ within dimensional regularization as we have shown in section 6.5.

Note however, that the operator $\chi\left(X\left[\phi_{*}\right](x)\right)$ is $\phi_{*}$ dependent and nonlocal, namely, because

$$
X\left[\phi_{*}\right](x)=x+2 \theta\left(\partial \phi_{*}\right)_{\theta}(x)
$$

and thus

$$
\chi\left(X\left[\phi_{*}\right](x)\right)=\sum_{n=0}^{\infty} \frac{(2 \theta)^{n}}{n !}\left(\partial^{\mu_{1}} \phi_{*}\right)_{\theta}(x) \ldots\left(\partial^{\mu_{n}} \phi_{*}\right)_{\theta}(x)\left(\partial_{\mu_{1}} \ldots \partial_{\mu_{n}} \chi\right)(x)
$$

Therefore $\chi\left(X\left[\phi_{*}\right](x)\right)$ is an infinite linear combinations of local operators $\left(\partial_{\mu_{1}} \ldots \partial_{\mu_{n}} \chi\right)(x)$ with $x$-dependent coefficients i.e. it is not translation invariant. The latter fact is the reason why we cannot use (C.7) to argue that also $S$ matrices for fluctuations are the same in both theories (cf. section 6.5).

\section{The group velocity of the plane wave perturbation}

The group velocity can be obtained by means of differentiation of condition (6.103) for the center of the wave packet (here $\widehat{\mathbf{k}}=\mathbf{k} /|\mathbf{k}|$ )

$$
\mathbf{X}\left[\phi_{*}\right]-\widehat{\mathbf{k}} X^{0}\left[\phi_{*}\right]=\text { const. }
$$

with respect to $t$, the group velocity is then $\mathbf{v}_{\text {group }}(x)=\mathrm{d} \mathbf{x} / \mathrm{d} t$. Explicitly we get

$$
X\left[\phi_{*}\right](x)=x+2 \theta n F^{\prime}(n \cdot x),
$$

and thus writing $n=(1, \mathbf{n})$

$$
\mathbf{v}_{\text {group }}+2 \theta \mathbf{n} F^{\prime \prime}(n \cdot x)\left(1-\mathbf{n} \cdot \mathbf{v}_{\text {group }}\right)-\widehat{\mathbf{k}}\left[1+2 \theta F^{\prime \prime}(n \cdot x)\left(1-\mathbf{n} \cdot \mathbf{v}_{\text {group }}\right)\right]=0
$$

and therefore denoting $v_{\|} \equiv\left(\mathbf{n} \cdot \mathbf{v}_{\text {group }}\right)$ the component of $\mathbf{v}_{\text {group }}$ parallel to $\mathbf{n}$

$$
v_{\|}+2 \theta F^{\prime \prime}(n \cdot x)\left(1-v_{\|}\right)-\mathbf{n} \cdot \widehat{\mathbf{k}}\left[1+2 \theta F^{\prime \prime}(n \cdot x)\left(1-v_{\|}\right)\right]=0 .
$$


As a result

$$
v_{\|}=\frac{\mathbf{n} \cdot \widehat{\mathbf{k}}-2 \theta F^{\prime \prime}(n \cdot x)(1-\mathbf{n} \cdot \widehat{\mathbf{k}})}{1-2 \theta F^{\prime \prime}(n \cdot x)(1-\mathbf{n} \cdot \widehat{\mathbf{k}})}
$$

Inserting this to (D.3) we get finally

$$
\begin{aligned}
\mathbf{v}_{\text {group }} & =\widehat{\mathbf{k}}\left[1+2 \theta F^{\prime \prime}(n \cdot x)\left(1-v_{\|}\right)\right]-2 \theta \mathbf{n} F^{\prime \prime}(n \cdot x)\left(1-v_{\|}\right) \\
& =\frac{\widehat{\mathbf{k}}-2 \theta \mathbf{n} F^{\prime \prime}(n \cdot x)(1-\mathbf{n} \cdot \widehat{\mathbf{k}})}{1-2 \theta F^{\prime \prime}(n \cdot x)(1-\mathbf{n} \cdot \widehat{\mathbf{k}})}
\end{aligned}
$$

\section{E Perturbative calculation of the two-point amplitude}

Here we explicitly calculate the first two perturbative contributions to the amplitude $\mathcal{M}\left(\mathbf{k}, \mathbf{k}^{\prime}\right)$. The first order contribution corresponds to the first graph on the right hand side of the graphical equation in the last row of the figure 3 and we get it simply by setting $p \rightarrow k, q \rightarrow-k^{\prime}$ in the Feynman rule for interaction vertex and putting the external lines on shell. That means, we get for the first order contribution to the $S$ matrix

$$
S_{f i}^{(1)}\left(\mathbf{k}, \mathbf{k}^{\prime}\right)=-4 \mathrm{i} \theta_{*}\left(k^{+}\right)^{2}(2 \pi)^{3} \delta\left(k^{+}-k^{+\prime}\right) \delta^{(2)}\left(\mathbf{k}_{\perp}-\mathbf{k}_{\perp}^{\prime}\right) \int \mathrm{d} x^{+} \mathrm{e}^{-\frac{i}{2}\left(k^{-}-k^{-\prime}\right) x^{+}} F^{\prime \prime}\left(x^{+}\right) .
$$

Provided $k$ and $k^{\prime}$ are on shell, i.e. $k^{-}=\mathbf{k}_{\perp}^{2} / k^{+}$and similarly for $k^{\prime}$, the exponential factor in the integrand is just one and we get (let us remind $\psi^{ \pm}=\lim _{x^{+} \rightarrow \pm \infty} F^{\prime}\left(x^{+}\right)$)

$$
S_{f i}^{(1)}\left(\mathbf{k}, \mathbf{k}^{\prime}\right)=\left[2 \mathrm{i} \theta_{*} k^{+}\left(\psi^{-}-\psi^{+}\right)\right] 2 k^{+}(2 \pi)^{3} \delta\left(k^{+}-k^{+\prime}\right) \delta^{(2)}\left(\mathbf{k}_{\perp}-\mathbf{k}_{\perp}^{\prime}\right)\left(\psi^{-}-\psi^{+}\right) .
$$

Using the identity valid for on-shell $k$

$$
\delta\left(k^{+}-k^{+\prime}\right) \delta^{(2)}\left(\mathbf{k}_{\perp}-\mathbf{k}_{\perp}^{\prime}\right)=\frac{|\mathbf{k}|}{k^{+}} \delta^{(3)}\left(\mathbf{k}-\mathbf{k}^{\prime}\right)=\frac{1}{k^{+}|\mathbf{k}|} \delta\left(|\mathbf{k}|-\left|\mathbf{k}^{\prime}\right|\right) \delta^{(2)}\left(\widehat{\mathbf{k}}-\widehat{\mathbf{k}}^{\prime}\right)
$$

we get finally

$$
\mathcal{M}^{(1)}\left(\mathbf{k}, \mathbf{k}^{\prime}\right)=(4 \pi)^{2} \frac{2 \mathrm{i} \theta_{*} k^{+}\left(\psi^{-}-\psi^{+}\right)}{2 \mathrm{i}|\mathbf{k}|} \delta^{(2)}\left(\widehat{\mathbf{k}}-\widehat{\mathbf{k}}^{\prime}\right) .
$$

The next term corresponding to the graph with two vertices and one propagator (see the second graph on the right hand side in the last row of figure 3) gives according the standard Feynman rules

$$
\begin{aligned}
& S_{f i}^{(2)}\left(\mathbf{k}, \mathbf{k}^{\prime}\right)= \\
= & \int \frac{\mathrm{d}^{4} p}{(2 \pi)^{4}} \frac{\mathrm{i}}{p^{+} p^{-}-\mathbf{p}_{\perp}^{2}+\mathrm{i} 0} \\
& \times\left[-4 \mathrm{i} \theta_{*}\left(k^{+} p^{+}\right)(2 \pi)^{3} \delta\left(k^{+}-p^{+}\right) \delta^{(2)}\left(\mathbf{k}_{\perp}-\mathbf{p}_{\perp}\right) \int \mathrm{d} x^{+} \mathrm{e}^{-\frac{i}{2}\left(p^{-}-k^{-}\right) x^{+}} F^{\prime \prime}\left(x^{+}\right)\right] \\
& \times\left[-4 \mathrm{i} \theta_{*}\left(k^{+\prime} p^{+}\right)(2 \pi)^{3} \delta\left(k^{+\prime}-p^{+}\right) \delta^{(2)}\left(\mathbf{k}_{\perp}^{\prime}-\mathbf{p}_{\perp}\right) \int \mathrm{d} y^{+} \mathrm{e}^{+\frac{i}{2}\left(p^{-}-k^{-\prime}\right) x^{+}} F^{\prime \prime}\left(y^{+}\right)\right] .
\end{aligned}
$$


Writing $\mathrm{d}^{4} p=(1 / 2) \mathrm{d} p^{-} \mathrm{d} p^{+} \mathrm{d}^{2} \mathbf{p}_{\perp}$ and integrating out the delta functions we get

$$
\begin{aligned}
S_{f i}^{(2)}\left(\mathbf{k}, \mathbf{k}^{\prime}\right)= & 8\left(\mathrm{i} \theta_{*}\right)^{2}\left(k^{+}\right)^{4}(2 \pi)^{3} \delta\left(k^{+}-k^{+\prime}\right) \delta^{(2)}\left(\mathbf{k}_{\perp}-\mathbf{k}_{\perp}^{\prime}\right) \\
& \times \int \mathrm{d} x^{+} \mathrm{d} y^{+} F^{\prime \prime}\left(x^{+}\right) F^{\prime \prime}\left(y^{+}\right) \\
& \times \int \frac{\mathrm{d} p^{-}}{2 \pi} \mathrm{e}^{-\frac{i}{2}\left(p^{-}-k^{-}\right)\left(x^{+}-y^{+}\right)} \frac{\mathrm{i}}{k^{+} p^{-}-\mathbf{k}_{\perp}^{2}+\mathrm{i} 0} .
\end{aligned}
$$

Using the on-shell condition $\mathbf{k}_{\perp}^{2}=k^{-} k^{+}$and shifting the integration variable $p^{-} \rightarrow p^{-}+k^{-}$ we can rewrite this as (note that $k^{+}>0$ )

$$
\begin{aligned}
S_{f i}^{(2)}\left(\mathbf{k}, \mathbf{k}^{\prime}\right)= & 8\left(\mathrm{i} \theta_{*}\right)^{2}\left(k^{+}\right)^{3}(2 \pi)^{3} \delta\left(k^{+}-k^{+\prime}\right) \delta^{(2)}\left(\mathbf{k}_{\perp}-\mathbf{k}_{\perp}^{\prime}\right) \\
& \times \int \mathrm{d} x^{+} \mathrm{d} y^{+} F^{\prime \prime}\left(x^{+}\right) F^{\prime \prime}\left(y^{+}\right) \int \frac{\mathrm{d} p^{-}}{2 \pi} \mathrm{e}^{-\frac{1}{2} p^{-}\left(x^{+}-y^{+}\right)} \frac{\mathrm{i}}{p^{-}+\mathrm{i} 0}
\end{aligned}
$$

We can recognize the integral representation of the Heaviside theta function in the last line, i.e.

$$
\begin{aligned}
S_{f i}^{(2)}\left(\mathbf{k}, \mathbf{k}^{\prime}\right)= & \left(2 \mathrm{i} \theta_{*} k^{+}\right)^{2} 2 k^{+}(2 \pi)^{3} \delta\left(k^{+}-k^{+\prime}\right) \delta^{(2)}\left(\mathbf{k}_{\perp}-\mathbf{k}_{\perp}^{\prime}\right) \\
& \times \int \mathrm{d} x^{+} \mathrm{d} y^{+} F^{\prime \prime}\left(x^{+}\right) F^{\prime \prime}\left(y^{+}\right) \theta\left(x^{+}-y^{+}\right) .
\end{aligned}
$$

The remaining integral is elementary and we get finally

$$
S_{f i}^{(2)}\left(\mathbf{k}, \mathbf{k}^{\prime}\right)=\frac{1}{2 !}\left[2 \mathrm{i} \theta_{*} k^{+}\left(\psi^{-}-\psi^{+}\right)\right]^{2} 2 k^{+}(2 \pi)^{3} \delta\left(k^{+}-k^{+\prime}\right) \delta^{(2)}\left(\mathbf{k}_{\perp}-\mathbf{k}_{\perp}^{\prime}\right)
$$

and

$$
\mathcal{M}^{(2)}\left(\mathbf{k}, \mathbf{k}^{\prime}\right)=(4 \pi)^{2} \frac{\left(2 \mathrm{i} \theta_{*} k^{+}\left(\psi^{-}-\psi^{+}\right)\right)^{2} / 2 !}{2 \mathrm{i}|\mathbf{k}|} \delta^{(2)}\left(\widehat{\mathbf{k}}-\widehat{\mathbf{k}}^{\prime}\right)
$$

The sum $\mathcal{M}^{(1)}+\mathcal{M}^{(2)}$ thus reproduces the first two terms of the expansion of the complete amplitude (6.124) in powers of the phase shift.

\section{F The fluctuation operator of the static cylindrically symmetric solution}

The fluctuation operator is

$$
\frac{\delta^{2} S_{\theta}[\phi]}{\delta \phi(x) \delta \phi(y)}=\sum_{n=2}^{d} n d_{n}(\theta) \frac{\partial \mathcal{L}_{n-1}^{\mathrm{der}}(\partial \partial \phi(x))}{\partial\left(\partial_{\mu} \partial_{\nu} \phi(x)\right)} \partial_{\mu} \partial_{\nu} \delta^{(d)}(x-y)
$$

From the generating function of $\mathcal{L}_{k}^{\text {der }}(\partial \partial \phi(x))$

$$
4 ! \operatorname{det}(\eta+w \partial \partial \phi)=\sum_{n=0}^{d} w^{k}\left(\begin{array}{l}
4 \\
k
\end{array}\right) \mathcal{L}_{k}^{\text {der }}(\partial \partial \phi)
$$

we get

$$
\frac{\partial}{\partial\left(\partial_{\mu} \partial_{\nu} \phi\right)} \operatorname{det}(\eta+w \partial \partial \phi)=\frac{1}{4 !} \sum_{n=0}^{d} w^{k}\left(\begin{array}{l}
4 \\
k
\end{array}\right) \frac{\partial \mathcal{L}_{k}^{\text {der }}(\partial \partial \phi)}{\partial\left(\partial_{\mu} \partial_{\nu} \phi\right)}
$$


Left hand side gives

$$
\begin{aligned}
\frac{\partial}{\partial\left(\partial_{\mu} \partial_{\nu} \phi\right)} \operatorname{det}(\eta+w \partial \partial \phi) & =-\frac{\partial}{\partial\left(\partial_{\mu} \partial_{\nu} \phi\right)} \operatorname{det}(\delta+w \eta \cdot \partial \partial \phi) \\
& =-\frac{\partial}{\partial\left(\partial_{\mu} \partial_{\nu} \phi\right)} \exp \operatorname{Tr} \ln (\delta+w \eta \cdot \partial \partial \phi) \\
& =\operatorname{det}(\eta+w \partial \partial \phi) \frac{\partial}{\partial\left(\partial_{\mu} \partial_{\nu} \phi\right)} \operatorname{Tr} \ln (\delta+w \eta \cdot \partial \partial \phi) \\
& =w \operatorname{det}(\eta+w \partial \partial \phi)\left[(\delta+w \eta \cdot \partial \partial \phi)^{-1}\right]_{\beta}^{\mu} \eta^{\nu \beta} \\
& =w \operatorname{det}(\eta+w \partial \partial \phi)\left[(\eta+w \partial \partial \phi)^{-1}\right]^{\mu \nu}
\end{aligned}
$$

For cylindrically symmetric static solution $\phi \equiv \phi(z \bar{z})$ we get

$$
\eta+w \partial \partial \phi=\left(\begin{array}{cccc}
1 & 0 & 0 & 0 \\
0 & -1 & 0 & 0 \\
0 & 0 & -1+w(\partial+\bar{\partial})^{2} \phi & w \mathrm{i}\left(\partial^{2}-\bar{\partial}^{2}\right) \phi \\
0 & 0 & w \mathrm{i}\left(\partial^{2}-\bar{\partial}^{2}\right) \phi & -1-w(\partial-\bar{\partial})^{2} \phi
\end{array}\right)
$$

and thus

$$
\operatorname{det}(\eta+w \partial \partial \phi)=-1+4 w \partial \bar{\partial} \phi+4 w^{2}\left[\partial^{2} \phi \bar{\partial}^{2} \phi-(\partial \bar{\partial} \phi)^{2}\right]
$$

and

$$
\begin{aligned}
\operatorname{det}(\eta+w \partial \partial \phi)(\eta+w \partial \partial \phi)^{-1} & \\
= & \left(\begin{array}{cccc}
\operatorname{det}(\eta+w \partial \partial \phi) & 0 & 0 & 0 \\
0 & -\operatorname{det}(\eta+w \partial \partial \phi) & 0 & 0 \\
0 & 0 & 1+w(\partial-\bar{\partial})^{2} \phi & w \mathrm{i}\left(\partial^{2}-\bar{\partial}^{2}\right) \phi \\
0 & 0 & w \mathrm{i}\left(\partial^{2}-\bar{\partial}^{2}\right) \phi & 1-w(\partial+\bar{\partial})^{2} \phi
\end{array}\right)
\end{aligned}
$$

As a result

$$
\begin{aligned}
& \frac{1}{4 !} \sum_{n=1}^{d} w^{k}\left(\begin{array}{l}
4 \\
k
\end{array}\right) \frac{\partial \mathcal{L}_{k}^{\operatorname{der}}(\partial \partial \phi)}{\partial(\partial \partial \phi)} \\
= & w\left(\begin{array}{cccc}
\operatorname{det}(\eta+w \partial \partial \phi) & 0 & 0 & 0 \\
0 & -\operatorname{det}(\eta+w \partial \partial \phi) & 0 & 0 \\
0 & 0 & 1+w(\partial-\bar{\partial})^{2} \phi & w \mathrm{i}\left(\partial^{2}-\bar{\partial}^{2}\right) \phi \\
0 & 0 & w \mathrm{i}\left(\partial^{2}-\bar{\partial}^{2}\right) \phi & 1-w(\partial+\bar{\partial})^{2} \phi
\end{array}\right)
\end{aligned}
$$


and thus

$$
\begin{aligned}
\frac{\partial \mathcal{L}_{1}^{\text {der }}(\partial \partial \phi)}{\partial\left(\partial_{\mu} \partial_{\nu} \phi\right)}= & -6 \eta \\
\frac{\partial \mathcal{L}_{2}^{\text {der }}(\partial \partial \phi)}{\partial\left(\partial_{\mu} \partial_{\nu} \phi\right)} & =4\left(\begin{array}{cccc}
4 \partial \bar{\partial} \phi & 0 & 0 & 0 \\
0 & -4 \partial \bar{\partial} \phi & 0 & 0 \\
0 & 0 & (\partial-\bar{\partial})^{2} \phi & \mathrm{i}\left(\partial^{2}-\bar{\partial}^{2}\right) \phi \\
0 & 0 & \mathrm{i}\left(\partial^{2}-\bar{\partial}^{2}\right) \phi-(\partial+\bar{\partial})^{2} \phi
\end{array}\right) \\
\frac{\partial \mathcal{L}_{3}^{\text {der }}(\partial \partial \phi)}{\partial\left(\partial_{\mu} \partial_{\nu} \phi\right)} & =6\left(\begin{array}{cccc}
4\left[\partial^{2} \phi \bar{\partial}^{2} \phi-(\partial \bar{\partial} \phi)^{2}\right] & 0 & 0 \\
0 & -4\left[\partial^{2} \phi \bar{\partial}^{2} \phi-(\partial \bar{\partial} \phi)^{2}\right] & 0 & 0 \\
0 & 0 & 0 & 0 \\
0 & 0 & 0
\end{array}\right)
\end{aligned}
$$

Inserting this to the formula

$$
g[\phi]^{\mu \nu}=\sum_{n=2}^{d} n d_{n}(\theta) \frac{\partial \mathcal{L}_{n-1}^{\mathrm{der}}(\partial \partial \phi(x))}{\partial\left(\partial_{\mu} \partial_{\nu} \phi(x)\right)}
$$

gives (6.126).

\section{G Full form of 4-pt scattering amplitude and self-energy}

The corresponding contributions are

$$
\begin{aligned}
& A_{1}=d_{3}^{4} \frac{B(s)}{(4 \pi)^{2}} \frac{81 s^{4}\left[\left(d^{2}+6 d+32\right) s^{2}-72 t u\right]}{32\left(d^{2}-1\right)}+\mathrm{cycl} \\
& A_{2}=-d_{3}^{4} \frac{B(s)}{(4 \pi)^{2}} \frac{81(d+2) s^{6}}{4(d-1)}+\mathrm{cycl} \\
& A_{3}=d_{3}^{4} \frac{B(s)}{(4 \pi)^{2}} \frac{81 s^{6}}{2}+\mathrm{cycl} \\
& A_{4}=d_{4}^{2} \frac{B(s)}{(4 \pi)^{2}} \frac{9 s^{4}\left[\left(d^{2}-2 d\right) s^{2}-8 t u\right]}{8\left(d^{2}-1\right)}+\mathrm{cycl} \\
& A_{5}=d_{3}^{2} d_{4} \frac{B(s)}{(4 \pi)^{2}} \frac{27 s^{4}\left[(d+4)(d-2) s^{2}+24 t u\right]}{8\left(d^{2}-1\right)}+\mathrm{cycl} \\
& A_{6}=-d_{3}^{2} d_{4} \frac{B(s)}{(4 \pi)^{2}} \frac{27 s^{6}(d-2)}{2(d-1)}+\mathrm{cycl}
\end{aligned}
$$

We have used the cyclic summation over all Mandelstam variables (e.g. $\left(s^{2}+\right.$ cycl $)=$ $\left.s^{2}+t^{2}+u^{2}\right)$. The loop function is given by

$$
B(s)=\frac{1}{(4 \pi)^{d / 2-2}} \frac{1}{d-3} \Gamma(2-d / 2) s^{d / 2-2}
$$

Summing up all diagrams leads to

$$
A=\left(d_{4}-\frac{9}{2} d_{3}^{2}\right)^{2} \frac{B(s)}{(4 \pi)^{2}} \frac{9 s^{4}\left[d(d-2) s^{2}-8 t u\right]}{8\left(d^{2}-1\right)}+\text { cycl. }
$$


The full result for the one-loop self-energy reads

$$
\Sigma(p)=-\frac{1}{(4 \pi)^{d / 2}} \frac{9}{2} d_{3}^{2}\left(p^{2}\right)^{4} B\left(p^{2}\right) .
$$

Open Access. This article is distributed under the terms of the Creative Commons Attribution License (CC-BY 4.0), which permits any use, distribution and reproduction in any medium, provided the original author(s) and source are credited.

\section{References}

[1] G.R. Dvali, G. Gabadadze and M. Porrati, $4-D$ gravity on a brane in $5-D$ Minkowski space, Phys. Lett. B 485 (2000) 208 [hep-th/0005016] [InSPIRE].

[2] C. Deffayet, G.R. Dvali and G. Gabadadze, Accelerated universe from gravity leaking to extra dimensions, Phys. Rev. D 65 (2002) 044023 [astro-ph/0105068] [INSPIRE].

[3] C. de Rham, G. Gabadadze and A.J. Tolley, Resummation of Massive Gravity, Phys. Rev. Lett. 106 (2011) 231101 [arXiv: 1011.1232] [INSPIRE].

[4] A. Nicolis, R. Rattazzi and E. Trincherini, The Galileon as a local modification of gravity, Phys. Rev. D 79 (2009) 064036 [arXiv:0811.2197] [inSPIRE].

[5] A.I. Vainshtein, To the problem of nonvanishing gravitation mass, Phys. Lett. B 39 (1972) 393 [INSPIRE].

[6] G.W. Horndeski, Second-order scalar-tensor field equations in a four-dimensional space, Int. J. Theor. Phys. 10 (1974) 363 [inSPIRE].

[7] C. Deffayet, S. Deser and G. Esposito-Farese, Generalized Galileons: All scalar models whose curved background extensions maintain second-order field equations and stress-tensors, Phys. Rev. D 80 (2009) 064015 [arXiv:0906.1967] [INSPIRE].

[8] C. Deffayet, S. Deser and G. Esposito-Farese, Arbitrary p-form Galileons, Phys. Rev. D 82 (2010) 061501 [arXiv: 1007.5278] [INSPIRE].

[9] C. de Rham and A.J. Tolley, DBI and the Galileon reunited, JCAP 05 (2010) 015 [arXiv: 1003.5917] [INSPIRE].

[10] J. Khoury, Les Houches Lectures on Physics Beyond the Standard Model of Cosmology, arXiv:1312.2006 [INSPIRE].

[11] T.L. Curtright and D.B. Fairlie, A Galileon Primer, arXiv:1212.6972 [INSPIRE].

[12] C. Deffayet and D.A. Steer, A formal introduction to Horndeski and Galileon theories and their generalizations, Class. Quant. Grav. 30 (2013) 214006 [arXiv:1307.2450] [InSPIRE].

[13] M.A. Luty, M. Porrati and R. Rattazzi, Strong interactions and stability in the DGP model, JHEP 09 (2003) 029 [hep-th/0303116] [INSPIRE].

[14] K. Hinterbichler, M. Trodden and D. Wesley, Multi-field galileons and higher co-dimension branes, Phys. Rev. D 82 (2010) 124018 [arXiv: 1008.1305] [INSPIRE].

[15] C. de Rham, G. Gabadadze, L. Heisenberg and D. Pirtskhalava, Nonrenormalization and naturalness in a class of scalar-tensor theories, Phys. Rev. D 87 (2013) 085017 [arXiv: 1212.4128] [INSPIRE]. 
[16] N. Brouzakis, A. Codello, N. Tetradis and O. Zanusso, Quantum corrections in Galileon theories, Phys. Rev. D 89 (2014) 125017 [arXiv:1310.0187] [inSPIRE].

[17] M. Fasiello and A.J. Tolley, Cosmological Stability Bound in Massive Gravity and Bigravity, JCAP 12 (2013) 002 [arXiv: 1308.1647] [INSPIRE].

[18] C. de Rham, M. Fasiello and A.J. Tolley, Galileon Duality, Phys. Lett. B 733 (2014) 46 [arXiv: 1308.2702] [INSPIRE].

[19] G. Goon, K. Hinterbichler, A. Joyce and M. Trodden, Galileons as Wess-Zumino Terms, JHEP 06 (2012) 004 [arXiv: 1203.3191] [INSPIRE].

[20] K. Kampf, J. Novotny and J. Trnka, Recursion Relations for Tree-level Amplitudes in the SU(N) Non-linear $\sigma$-model, Phys. Rev. D 87 (2013) 081701 [arXiv:1212.5224] [InSPIRE].

[21] F.A. Berends and W.T. Giele, Recursive Calculations for Processes with n Gluons, Nucl. Phys. B 306 (1988) 759 [INSPIRE].

[22] S.R. Coleman, J. Wess and B. Zumino, Structure of phenomenological Lagrangians. 1., Phys. Rev. 177 (1969) 2239 [INSPIRE].

[23] C.G. Callan Jr., S.R. Coleman, J. Wess and B. Zumino, Structure of phenomenological Lagrangians. 2., Phys. Rev. 177 (1969) 2247 [INSPIRE].

[24] D.V. Volkov, Phenomenological Lagrangians, Fiz. Elem. Chast. Atom. Yadra 4 (1973) 3.

[25] V. Ogievetsky, Nonlinear realizations of internal and spacetime symmetries, Acta Universitatis Wratislaviensis 207 (1974) 117.

[26] E.A. Ivanov and V.I. Ogievetsky, The Inverse Higgs Phenomenon in Nonlinear Realizations, Teor. Mat. Fiz. 25 (1975) 164 [INSPIRE].

[27] J. Wess and B. Zumino, Consequences of anomalous Ward identities, Phys. Lett. B 37 (1971) 95 [INSPIRE].

[28] E. Witten, Global Aspects of Current Algebra, Nucl. Phys. B 223 (1983) 422 [InSPIRE].

[29] E. D'Hoker and S. Weinberg, General effective actions, Phys. Rev. D 50 (1994) 6050 [hep-ph/9409402] [INSPIRE].

[30] E. D'Hoker, Invariant effective actions, cohomology of homogeneous spaces and anomalies, Nucl. Phys. B 451 (1995) 725 [hep-th/9502162] [INSPIRE].

[31] H. Watanabe and T. Brauner, On the number of Nambu-Goldstone bosons and its relation to charge densities, Phys. Rev. D 84 (2011) 125013 [arXiv:1109.6327] [INSPIRE].

[32] T. Brauner and H. Watanabe, Spontaneous breaking of spacetime symmetries and the inverse Higgs effect, Phys. Rev. D 89 (2014) 085004 [arXiv:1401.5596] [INSPIRE].

[33] I. Low and A.V. Manohar, Spontaneously broken space-time symmetries and Goldstone's theorem, Phys. Rev. Lett. 88 (2002) 101602 [hep-th/0110285] [INSPIRE].

[34] I.N. McArthur, Nonlinear realizations of symmetries and unphysical Goldstone bosons, JHEP 11 (2010) 140 [arXiv:1009.3696] [INSPIRE].

[35] J.A. de Azcarraga, A.J. Macfarlane and J.C. Perez Bueno, Effective actions, relative cohomology and Chern Simons forms, Phys. Lett. B 419 (1998) 186 [hep-th/9711064] [INSPIRE]. 
[36] J.A. de Azcarraga, J.M. Izquierdo and J.C. Perez Bueno, An Introduction to some novel applications of Lie algebra cohomology in mathematics and physics, Rev. R. Acad. Cien. Exactas Fis. Nat. Ser. A Mat. 95 (2001) 225 [physics/9803046] [InSPIRE].

[37] C. de Rham, Massive Gravity, Living Rev. Rel. 17 (2014) 7 [arXiv:1401.4173] [InSPIRE].

[38] C. de Rham, L. Keltner and A.J. Tolley, Generalized Galileon Duality, Phys. Rev. D 90 (2014) 024050 [arXiv: 1403.3690] [INSPIRE].

[39] S. Deser and J. Franklin, Symmetrically reduced Galileon equations and solutions, Phys. Rev. D 86 (2012) 047701 [arXiv:1206.3217] [INSPIRE].

[40] P. Creminelli, M. Serone, G. Trevisan and E. Trincherini, Inequivalence of Coset Constructions for Spacetime Symmetries, arXiv:1403.3095 [INSPIRE].

[41] G. Barnich, M. Henneaux and C. Schomblond, On the covariant description of the canonical formalism, Phys. Rev. D 44 (1991) 939 [INSPIRE].

[42] E. Babichev, V. Mukhanov and A. Vikman, $k$-Essence, superluminal propagation, causality and emergent geometry, JHEP 02 (2008) 101 [arXiv:0708.0561] [INSPIRE].

[43] J. Evslin and T. Qiu, Closed Timelike Curves in the Galileon Model, JHEP 11 (2011) 032 [arXiv: 1106.0570] [InSPIRE].

[44] J. Gasser and H. Leutwyler, Chiral Perturbation Theory to One Loop, Annals Phys. 158 (1984) 142 [INSPIRE].

[45] J. Gasser and H. Leutwyler, Chiral Perturbation Theory: Expansions in the Mass of the Strange Quark, Nucl. Phys. B 250 (1985) 465 [InSPIRE].

[46] S. Weinberg, Phenomenological Lagrangians, Physica A 96 (1979) 327 [InSPIRE].

[47] T. de Paula Netto and I.L. Shapiro, One-loop divergences in the Galileon model, Phys. Lett. B 716 (2012) 454 [arXiv: 1207.0534] [INSPIRE]. 